\title{
A Mean Field Game Theoretic Approach for Security Enhancements in Mobile Ad-hoc Networks
}

\author{
by \\ Yanwei Wang

\begin{abstract}
A dissertation submitted to the
Faculty of Graduate Studies and Research
\end{abstract} \\ in partial fulfillment of the requirements for the degree of
}

\begin{abstract}
Master of Applied Science in Electrical and Computer Engineering
Ottawa-Carleton Institute for Electrical and Computer Engineering (OCIECE)

Department of Systems and Computer Engineering

Carleton University

Ottawa, Ontario, Canada, K1S 5B6

July, 2014
\end{abstract}

(C)Copyright 2014, Yanwei Wang 
The undersigned hereby recommends to the

Faculty of Graduate Studies and Research

acceptance of the dissertation

\title{
A Mean Field Game Theoretic Approach for Security Enhancements in Mobile Ad-hoc Networks
}

\author{
submitted by \\ Yanwei Wang, M.A.Sc. \\ in partial fulfillment of the requirements for the degree of \\ Master of Applied Science in Electrical and Computer Engineering
}

Prof. Fei Richard Yu, SCE, Carleton, Thesis Supervisor

Chair, Prof. Roshdy Hafez,

Department of Systems and Computer Engineering

Carleton University

July 2014 


\section{Abstract}

Game theory can provide a useful tool to study the security problem in mobile adhoc networks (MANETs). In this thesis, we propose the mean field game theory for security in MANETs, which can provide a powerful mathematical tool for problems with a large number of players. To the best of our knowledge, using mean field game theoretic approach for security in MANETs has not been considered in the existing works. The proposed scheme can enable an individual node in MANETs to make strategic security defence decisions without centralized administration. In addition, since security defence mechanisms consume precious system resources (e.g., energy), the proposed scheme considers not only the security requirement of MANETs but also the system resources. Moreover, each node in the proposed scheme only needs to know its own state information and the aggregate effect of the other nodes in MANETs. Therefore, the proposed scheme is a fully distributed scheme.

In addition, we consider a specific kind of MANETs, cognitive radio mobile ad-hoc networks (CR-MANETs), which have attracted great interests from both academia and industry. We propose a dynamic mean field game theoretic approach for security problems in CR-MANETs. The mean field game theoretic approach is proposed to enable an individual node in CR-MANETs to make strategic decisions for secu-

rity defence without centralized administration. Simulation results are presented to illustrate the effectiveness of the proposed scheme. 
To my parents, my wife and son. 


\section{Acknowledgments}

The author wishes to express his sincere appreciation to his supervisor, Dr. F. Richard Yu. He is grateful to his support, encouragement and invaluable advice.

Special thanks are due to Dr. Minyi Huang and Dr. Helen Tang for their valuable advice. The author expresses his thanks to his colleagues Mr. Yegui Cai, Mr. Zhexiong Wei, Mr. Zhiyuan Yin, Mr. Chengchao Liang, Dr. Shengrong Bu, Mr. Osman Abdillahi and the other graduate students in OCIECE for their assistance and happy time spent together.

Thanks are also due to the faculty and staff in Department of Systems and Computer Engineering for providing support to the author's graduate study and thesis experiment. 


\section{Table of Contents}

Abstract $\quad$ iii

Acknowledgments $\quad$ V

Table of Contents vi vi

List of Tables $\quad$ X

List of Figures $\quad$ xi

List of Abbreviations xiii

List of Symbols $\quad$ XV

1 Introduction 1

1.1 Research Overview ...................... 1

1.2 Research Motivations . . . . . . . . . . . . . . . . 2

1.3 Research Objectives . . . . . . . . . . . . . . . . 3

1.4 Thesis Contributions . . . . . . . . . . . . . . . . . 3

1.4.1 Published Papers .................... 4

1.5 Thesis Organization . . . . . . . . . . . . . . . . . 4

2 Background and Related Work $\quad 6$ 
2.1 Mobile Ad-hoc Networks . . . . . . . . . . . . . . 6

2.1.1 Development of Mobile Ad-hoc Networks . . . . . . . . . . 7

2.1.2 Characteristics of Mobile Ad-hoc Networks . . . . . . . . . . . 10

2.2 Security in Mobile Ad-hoc Networks . . . . . . . . . . . . . 11

2.3 Security in Vehicular Ad-hoc Networks . . . . . . . . . . . . . . . . . 12

2.4 Security in Cognitive Radio Mobile Ad-hoc Networks . . . . . . . . . 14

2.5 Game Theoretic Approaches for Security in MANETs . . . . . . . . . 16

2.5.1 Mean Field Game . . . . . . . . . . . . . . . . . . . . . . . 19

2.6 Summary . . . . . . . . . . . . . . . . . . . . . . 21

3 Proposed Mean Field Game Theoretic Approach 22

3.1 System Description . . . . . . . . . . . . . . . . . . 22

3.2 Model Description . . . . . . . . . . . . . . . . . 23

3.2.1 States, Transition Laws, and Cost Functions . . . . . . . . . 23

3.2.2 Mean Field Equation System . . . . . . . . . . . . 27

3.3 Approximation of the Mean Field Process . . . . . . . . . . . . . . . 29

3.3.1 Error Estimate on the Mean Field Approximation . . . . . . . 31

3.3.2 Cost Functions with the Approximation of Mean Field Process 33

3.3.3 Solution to Mean Field Equation System . . . . . . . . . . . . 34

3.4 Summary . . . . . . . . . . . . . . . . . . . 35

4 MANETs Simulation Results and Discussions 37

4.1 Distributed Optimal Defending Strategy in MANETs . . . . . . . . . 37

4.1.1 Mixed Strategies and State Transition Laws for the Major Player 39

4.1.2 Mixed Strategies and State Transition Laws for a Representative Minor Player . . . . . . . . . . . . . . . . 42

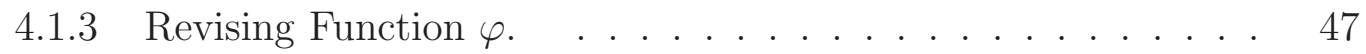


4.2 Simulation Results and Discussions . . . . . . . . . . . . 48

4.2.1 Simulation Scenarios . . . . . . . . . . . . . . 48

4.2.2 Average Cost . . . . . . . . . . . . . . . . . 49

4.2.3 Defence Actions According to Optimal Strategy . . . . . . . . 50

4.2.4 Performance with Limited Energy . . . . . . . . . . . . . . . 52

4.2.5 Performance with Sufficient Energy . . . . . . . . . . . . 54

4.3 Summary . . . . . . . . . . . . . . . . . . 56

5 Mean Field Game Method for Security in Cognitive Radio Mobile $\begin{array}{ll}\text { Ad-hoc Networks } & 57\end{array}$

5.1 Introduction . . . . . . . . . . . . . . . . 57

5.2 Model Description and Formulation in CR-MANETs . . . . . . . . 61

5.2.1 Description of System Model . . . . . . . . . . . . . . . 61

5.2.2 Energy Detection Spectrum Sensing Method . . . . . . . . . 62

5.2.3 Major Player and Minor Players in CR-MANETs . . . . . . . 64

5.2.4 States and Transition Laws in CR-MANETs . . . . . . . . . . 65

5.2.5 Cost Functions of CR-MANETs . . . . . . . . . . . 66

5.2.6 Mean Field Equation System . . . . . . . . . . . . 66

5.3 Approximation Method of the Mean Field Process . . . . . . . . . . . 67

5.3.1 Cost Functions with the Approximation of Mean Field Process 68

5.3.2 Mixed Strategies and State Transition Laws . . . . . . . . 69

5.4 Simulation Results and Discussions . . . . . . . . . . . . . . 72

5.4.1 Average Costs of Secondary Users . . . . . . . . . . . 73

5.4.2 Defence Actions According to Optimal Strategy . . . . . . . . 74

5.4.3 False Alarm Probability and Missing Detection Probability . . 75

5.5 Summary . . . . . . . . . . . . . . . . . . . . . 79 
6 Conclusions and Future Work $\quad 80$

6.1 Conclusions . . . . . . . . . . . . . . . . 80

6.2 Future Work . . . . . . . . . . . . . . . . 81

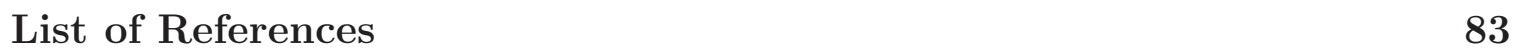

$\begin{array}{ll}\text { Appendix A Simulation Programs } & 90\end{array}$ 


\section{List of Tables}

1 Applications of Mobile Ad-hoc Networks . . . . . . . . . . . . . . . . 9

2 Setups of the Elements for Both the Major Player and the Representative Minor Player in MANETs . . . . . . . . . . . . . . . . . . 38

3 Utility Matrix of the Representative Minor Player $\mathcal{A}_{i}$ in MANETs . . 43

4 Setups of the Elements for Both the Major Player and the Representative Minor Player in CR-MANETs . . . . . . . . . . . . . . . . 69

5 Utility Matrix of the Representative Minor Player in CR-MANETs . 71 


\section{List of Figures}

1 A mobile ad-hoc network. . . . . . . . . . . . . . . 7

2 A $N+1$-node VANET with a malicious vehicle. . . . . . . . . 13

3 A $N$-node MANET with an attacker. . . . . . . . . . . . . 23

4 The mean field game model of a MANET with mixed players. $\left(x_{0}\right.$ : state of major player; $x_{i}, x_{j}$ : states of minor players $i$ and $j ; u_{0}$ : action of major player; $u_{i}$ and $u_{j}$ : actions of minor player $i$ and $j ; \rho_{i}$ and $\rho_{j}$ : weights of major player's action.) . . . . . . . . . . . . . 24

5 Value iteration for the major player. . . . . . . . . . . . . . 41

6 Value iteration for a representative minor player. . . . . . . . . . 45

7 Average cost comparison among the representative minor player with security-prioritized strategy, energy-prioritized strategy and optimal strategy (under the dynamical attack) . . . . . . . . . . . 49

8 Average cost comparison among the representative minor player with security-prioritized strategy, with energy-prioritized strategy and with optimal strategy (under the continuous attack). . . . . . . 50

9 Attacking target and defence action under major player's continuous

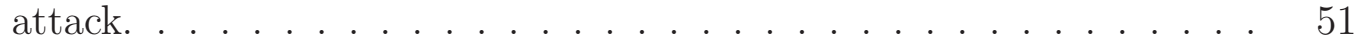

10 Attacking target and defence action under major player's dynamical

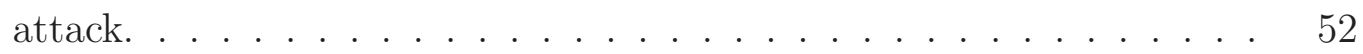


11 Comparison of average lifetime with different numbers of nodes (limited energy). . . . . . . . . . . . . . . . . . 53

12 Comparison of compromising probabilities with different numbers of nodes (limited energy). . . . . . . . . . . . . . . 54

13 Comparison of average lifetimes with different numbers of nodes (sufficient energy). . . . . . . . . . . . . . . . . . 55

14 Comparison of compromising probabilities with different numbers of nodes (sufficient energy). . . . . . . . . . . . . . . 55

15 Spectrum usage (from FCC Report [1]). . . . . . . . . . . . . . . 58

16 Four categories of the game-theoretic spectrum sharing approaches [1]. 60

17 System consists of a CR-MANET with $N$ nodes and an attacker. . . 61

18 Block diagram of an energy detector. . . . . . . . . . . . . 63

19 Average cost comparison among the representative minor player with security-prioritized strategy, energy-prioritized strategy and optimal strategy. . . . . . . . . . . . . . . . . .

20 Attacking target and defence action under major player's dynamical attack. . . . . . . . . . . . . . . . . 74

21 Missing detection probability and false alarm probability. . . . . . . 76

22 Missing detection probability $P_{m}$ versus false alarm probability $P_{f}$ (when secondary user has the same SNR, i.e., $\bar{\gamma}=10 d B$ ). . . . . . . 76

23 Missing detection probability versus average SNR $\left(P_{f}=10^{-1}, T W=5\right) .77$

24 False alarm probability comparison between the proposed scheme and the existing consensus-based scheme. . . . . . . . . . . . 78 


\section{List of Abbreviations}

AODV Ad-hoc on-demand distance vector

ALPHA Adaptive and Lightweight Protocol for Hop-by-hop Authentication

ALPHA-M ALPHA with Pre-signed Merkle Tree

AWGN Additive white Gaussian noise

BER Bit Error Rate

BPSK Binary Phase Shift Keying

CR Cognitive radio

CR-MANETs Cognitive radio mobile ad-hoc networks

DARPA Defense Advanced Research Projects Agency

DHCP Dynamic Host Configuration Protocol

DoS Denial of Service

FCC Federal Communications Commission

FEC Forward Error Correction

FSDF Fixed Selective Decode-and-Forward

GBN Go-Back-N

HEAP Hop-by-Hop Efficient Authentication Protocol

IDSs Intrusion detection systems

ITU International Telecommunications Union

MAC Message Authentication Code

MANET Mobile Ad-hoc Network

MIMO Multiple-Input Multiple-Output

PAN Personal area network 


$\begin{array}{ll}\text { PRNet } & \text { Packet Radio Network } \\ \text { PU } & \text { Primary user } \\ \text { QoS } & \text { Quality of Service } \\ \text { SNR } & \text { Signal-to-Noise Ratio } \\ \text { SSDF } & \text { Spectrum sensing data falsification } \\ \text { SU } & \text { Secondary user } \\ \text { VANETs } & \text { Vehicular Ad-hoc Networks } \\ \text { V2I } & \text { Vehicle-to-infrastructure } \\ \text { V2V } & \text { Vehicle-to-vehicle } \\ \text { WLAN } & \text { Wireless Local Area Network } \\ \text { WSN } & \text { Wireless Sensor Network }\end{array}$




\section{List of Symbols}

N

$S_{0}=\left\{1, \cdots, K_{0}\right\}$

$A_{0}=\left\{1, \cdots, L_{0}\right\}$

$S=\{1, \cdots, K\}$

$A=\{1, \cdots, L\}$

$x_{0}(t)$

$u_{0}(t)$

$x_{i}(t)$

$u_{i}(t)$

$\alpha_{\mathrm{E}_{0}}, \alpha_{I_{0}}$

$\alpha_{\mathrm{E}_{i}}, \alpha_{S_{i}}$

$I^{(N)}(t)$

$c_{0}\left(x_{0}, u_{0}, I^{(N)}\right)$

$f_{0}\left(x_{0}(t), u_{0}(t)\right)$

$f\left(I^{(N)}(t)\right)$

$c\left(x_{i}, u_{i}, x_{0}, u_{0}, I^{(N)}\right)$

$g_{i}\left(x_{i}(t), u_{i}(t)\right)$

$g_{0}^{i}\left(I^{(N)}(t), x_{0}(t), u_{0}(t)\right)$

$Q_{0}\left(z \mid y, a_{0}\right)$

$Q\left(z \mid y, a_{i}\right)$

$w_{i}$
The number of defending MANET nodes

The attacker's state space

The attacker's action space

The defenders' state space

The defenders' action space

The attacker $\mathcal{A}_{0}$ 's state at time $\mathrm{t}$

The attacker $\mathcal{A}_{0}$ 's action at time $\mathrm{t}$

The defender $\mathcal{A}_{i}$ 's state at time $\mathrm{t}$

The defender $\mathcal{A}_{i}$ 's action at time $\mathrm{t}$

The weights of $\mathcal{A}_{0}$ 's energy asset and information asset

The weights of $\mathcal{A}_{i}$ 's energy asset and security asset

The frequency of occurrence of the states in

the $N$-node MANET at time $t$

The cost of $\mathcal{A}_{0}$

The coupled energy cost of $\mathcal{A}_{0}$

The payoff of $\mathcal{A}_{0}$

The cost of a representative defender

The coupled energy cost of $\mathcal{A}_{i}$

The combined cost of $\mathcal{A}_{i}$

The state transition law of $\mathcal{A}_{0}$

The state transition law of $\mathcal{A}_{i}$

The minor player $\mathcal{A}_{i}$ 's security value 


\begin{tabular}{|c|c|}
\hline$\theta(t)$ & $\begin{array}{l}\text { The limiting process to approximate the random } \\
\text { measure process } I^{(N)}(t)\end{array}$ \\
\hline$\gamma_{i} \alpha_{i}-\left(1-\gamma_{i}\right) \beta_{i}$ & $\begin{array}{l}\text { The security value protected by the representative } \\
\text { minor player }\end{array}$ \\
\hline$\alpha_{i}$ & $\mathcal{A}_{i}$ 's security value with successfully defending \\
\hline$\beta_{i}$ & $\mathcal{A}_{i}$ 's loss of the security value with unsuccessfully defending \\
\hline$\gamma_{i}$ & The successful defending rate of $\mathcal{A}_{i}$ \\
\hline$\pi_{0}=\left(\rho_{1}, \rho_{2}, \cdots, \rho_{L_{0}}\right)$ & The major player's strategy \\
\hline$\rho_{K_{0}}$ & The probability of selecting an action $K_{0}$ \\
\hline$\pi=\left(\tau_{1}, \tau_{2}, \cdots, \tau_{L}\right)$ & The minor players' strategy \\
\hline$\tau_{K}$ & The probability of selecting an action $K$ \\
\hline$\theta(t+1)=\varphi\left(x_{0}(t), \theta(t)\right)$ & The updating rule for $\theta(t)$ \\
\hline$Q^{*}\left(x_{0}, \theta\right)$ & The $K \times K$ matrix to revise the $\varphi$ function \\
\hline$f_{s}$ & A center frequency for spectrum \\
\hline$W$ & The bandwidth of interest \\
\hline$T$ & The observation interval \\
\hline$X(t)$ & The signal received by the $\mathrm{SU}$ \\
\hline$S_{J}(t)$ & The intruder's jamming attack signal \\
\hline$n(t)$ & The additive white Gaussian noise \\
\hline$h$ & The amplitude gain of the channel \\
\hline$\gamma$ & The signal-to-noise ratio \\
\hline$X_{2 T W}^{2}$ & The random variable with central chi-square distributions \\
\hline$X_{2 T W}^{2}(2 \gamma)$ & The random variable with non-central chi-square distributions \\
\hline$T W$ & The time-bandwidth product \\
\hline$e_{(2 \bar{\gamma}+2)}$ & A random variable having the exponential distribution \\
\hline$\mu$ & The weight of the frequency of occurrence about the states \\
\hline
\end{tabular}




\section{Chapter 1}

\section{Introduction}

\section{$1.1 \quad$ Research Overview}

As a discipline aimed at modeling situations in which decision makers have to make specific actions, game theory can provide a powerful tool for the study of the security problem in wireless networks. However, most of the existing works on applying game theories to security only consider two players in the security game model: an attacker and a defender. This assumption may be valid for a network with centralized administration. But it is not realistic in MANETs or CR-MANETs, where centralized administration is not available. Consequently, each individual node in MANETs or CR-MANETs should be treated separately in the security game model. In this thesis, we propose a novel mean field game theoretic approach for security in MANETs and CR-MANETs. The mean field game theory provides a powerful mathematical tool for problems with a large number of players. To the best of our knowledge, using mean field game theoretic approach for security in MANETs or CR-MANETs has not been considered in existing works. The proposed scheme can enable an individual node in MANETs or CR-MANETs to make strategic security defence decisions without centralized administration. In addition, since security defence mechanisms 
consume precious system resources (e.g., energy), the proposed scheme considers not only the security requirement but also the system resources. Moreover, each node in the proposed scheme only needs to know its own state information and the aggregate effect of the other nodes in MANETs or CR-MANETs. Therefore, the proposed scheme is a fully distributed scheme. Simulation results are presented to illustrate the effectiveness of the proposed scheme.

\subsection{Research Motivations}

While wireless networking becomes almost omnipresent, security has become one of the key issues in the research field of MANETs and CR-MANETs. In a MANET or CR-MANET, mobile nodes can autonomously organize and communicate with each other over bandwidth-constrained wireless links. A wireless mobile node can function both as a network router for routing packets from the other nodes and as a network host for transmitting and receiving data. The topology of the networks changes dynamically and unpredictably because of nodes mobility. Many distributed algorithms have been investigated to determine the networking organization, routing, and link scheduling. On the other hand, the unique characteristics of these networks present some new challenges to security design due to the lack of any central authority and shared wireless medium [2]. There are various security threats that exist in MANETs or CR-MANETs, such as denial of service, black hole, resource consumption, location disclosure, wormhole, host impersonation, information disclosure, and interference $[3,4]$.

For solving the problems in MANETs or CR-MANETs which we mentioned above, an approach based on mean field game theory is proposed to model the interactions between the attacker and defenders. 


\subsection{Research Objectives}

The main objective of this research is to design a mean field game theoretic approach for security in mobile ad-hoc networks, which considers the trade-off both on system security and system resource consumption. More concisely, our objectives are shown as below:

- To propose a quantitative decision making approach, which is based on mean field game theory and which takes both security and resource consumption into consideration.

- To find an approximation method to overcome the fundamental complexity and enable an individual node to make strategic security defence decisions with high efficiency without centralized administration.

- To evaluate the proposed mean field game theoretic approach for security and resource consumption by comparing the simulation results obtained by applying the proposed approach with those achieved by applying the existing approaches.

\subsection{Thesis Contributions}

Based on the objectives mentioned above, we propose a mean field game theoret-

ic approach for security and system consumption in mobile ad-hoc networks. The following are the thesis contributions:

- A dynamic mean field game theoretic approach is proposed to enable an individual node in MANETs and CR-MANETs to make strategic security defence decisions without centralized administration.

- The proposed mean field game theoretic approach for security in MANETs and CR-MANETs tries to balance the system security and system resource 
consumption. The simulation results demonstrate that it can not only reduce the consumption of system resources, but also improve the networks' security.

\subsubsection{Published Papers}

The following papers have been published:

- Y. Wang, H. Tang, F. R. Yu, and M. Huang, "Mean field game theoretic approach for security in mobile ad-hoc networks", in Proc. SPIE, Vol. 8755, 875509, 2013.

- Y. Wang, F. R. Yu, M. Huang, A. Boukerche and T. Chen, "Securing Vehicular Ad-Hoc Networks with Mean Field Game Theory", in Proc. ACM DIVANet'13, no. 6, pp. 55-60, Barcelona, Spain, 2013.

- Y. Wang, F. R. Yu, H. Tang, and M. Huang, "A Mean Field Game Theoretic Approach for Security Enhancements in Mobile Ad-hoc Networks", IEEE Trans. Wireless Comm., vol. 13, no. 3, pp. 1616-1627, Mar 2014.

\subsection{Thesis Organization}

The rest of the thesis is organized as follows:

- Chapter 2 describes the background of the research which is presented in this thesis. The concepts of the mobile ad-hoc network communications are introduced, including the development and characteristics of mobile ad-hoc networks, the security issues in mobile ad-hoc networks, vehicle ad-hoc networks and cognitive radio ad-hoc networks, and the game theoretic approaches for security in mobile ad-hoc networks. 
- Chapter 3 presents the proposed mean field game theoretic approach for security and system resource consumption in mobile ad-hoc networks. The system which consists of a $N$-node MANET and an attacker is firstly described. The security problem of this system is formulated as an $N+1$ mean field game. The proposed game theoretic approach is discussed by setting up the system model and presenting the states, transition laws, and cost functions.

- Chapter 4 describes and discusses the simulation results of MANETs. When the proposed game theory approach is applied by a representative node, the average cost, the actions choosing and the system resource consumption in MANETs are introduced. The performances of a representative node adopting the optimal strategy are compared along with the performances of the node adopting other two strategies [5]. Additionally, in this chapter, we consider the situations of the nodes in MANET with limited energy and sufficient energy.

- Chapter 5 introduces the application of the proposed mean field game theoretic method for security problem in CR-MANETs. The jamming attack, which is loaded by a smart attacker, is considered in this research. The attacker tries to disrupt the communication among the secondary users. But when the primary user is using the spectrum, the attacker does not initiate the attack for the heavy punishment. This research considers a very critical situation for the tactical CR-MANETs.

- Chapter 6 wraps up the conclusions of this research and the future research orientation.

The simulation programs are presented in Appendix A. 


\section{Chapter 2}

\section{Background and Related Work}

\subsection{Mobile Ad-hoc Networks}

Ad-hoc networks are special kinds of wireless networks without any fixed infrastructure or centralized administration. As shown in Fig. 1, the basic units of a mobile ad-hoc network are called nodes or terminals. The nodes can work as routers in the network. So they can not only send or receive their own data, but also forward the traffic in the network.

In a mobile ad-hoc network, the nodes have wireless communications capability without the benefit of a mediating infrastructure [6]. Every node has the mobile capacity and can become aware of the presence of other nodes within its range. In this situation, these nodes which are found can be called neighbors because direct wireless communications links can be established between them [7]. Links established in the ad-hoc mode do not rely on the use of an access point of base station. Neighbors can communicate directly with each other. The nodes and links form a topology. Any pair of nodes, not directly connected, can communicate if there is a path, consisting of individual links, connecting them. Data units are routed through the path from the origin to the destination. Routing in the ad-hoc mode 


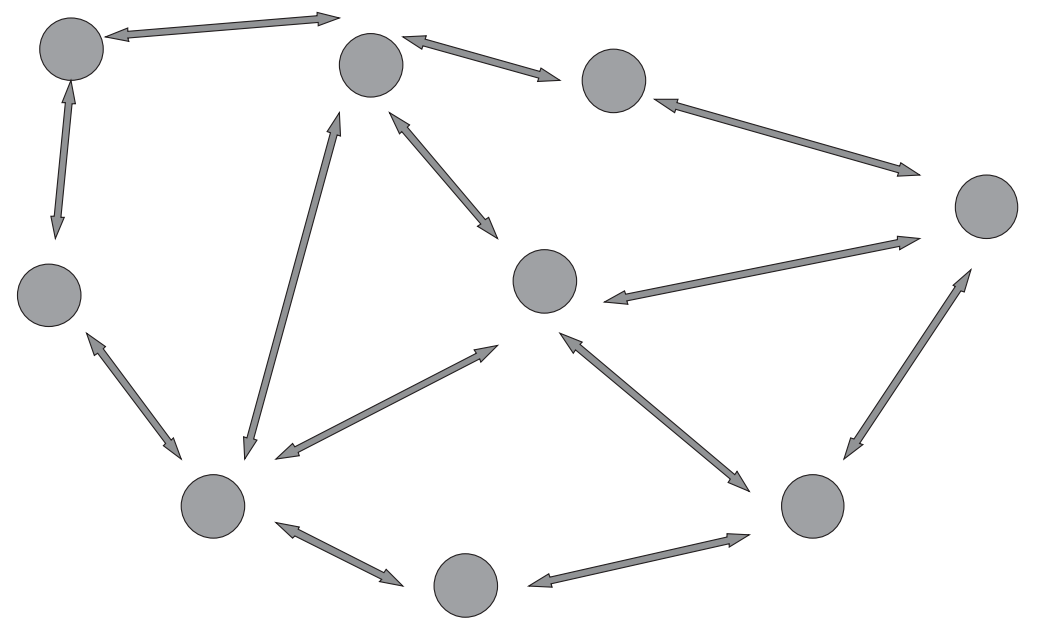

Figure 1: A mobile ad-hoc network.

means that there is no need for an address configuration server such as DHCP or routers. Every node autonomously configures its network address and can resolve the way to reach the destination, with the help from other nodes. Every node also plays an active role in forwarding data units for other nodes.

\subsubsection{Development of Mobile Ad-hoc Networks}

Propelled by web and multimedia applications, the usage of wireless networks has skyrocketed in the last decade. When shopping at the local department store, people can easily access the wireless networking to compare prices on the web.

Compared with the traditional mobile wireless networks, the mobile ad-hoc network does not rely on any fixed infrastructure. Mobile nodes can autonomously organize and communicate with each other. The wireless mobile node can function not only as a network host for transmitting and receiving the data, but also as a network router for routing packets from the other nodes.

Early ad-hoc networking applications can be traced to the Defense Advanced 
Research Projects Agency (DARPA) Packet Radio Network (PRNet) project in 1972 $[6,7]$. It was designed for the tactical network to improve battlefield communications and survivability. On the battlefield, it is dangerous to rely on access to a fixed preplaced communication infrastructure, since it's easy to be destroyed by the enemy. In this situation, a mobile ad-hoc network can provide a suitable framework and a mobile wireless distributed multi-hop network without any fixed infrastructure. Since this kind of flexible network can be set up anywhere at any time without infrastructure, the commercial potential and advantages of mobile ad-hoc networks have been realized by more and more people [6].

Although the early developments and applications of MANETs were supported and oriented by the military, now the nonmilitary MANET applications have grown substantially. Especially with the development of the new technologies, such as IEEE 802.11, Bluetooth and Hiperlan [7,8], the deployment of ad-hoc technology has been greatly promoted in the nonmilitary domain. For example, as a specific case of adhoc networks, Vehicular Ad-hoc Networks (VANETs), have attracted more and more researchers not only from academic, but also from industry all over the world $[9,10]$.

[6] introduced a classification of the present and future applications of mobile ad-hoc networks not only in the tactical networks, but also in the sensor networks, search-and-rescue operations, commercial and educational applications, personal area networking and so on. They are shown in Table 1.

There are many advantages in mobile ad-hoc networks:

- The devices themselves are the network.

- It allows seamless communication with very low cost.

- It works in a self-organized fashion and with easy deployment.

As opposed to dedicated nodes of a classical network, the nodes of an ad-hoc 
Table 1: Applications of Mobile Ad-hoc Networks

\begin{tabular}{|l|l|}
\hline Applications & Descriptions/ Services \\
\hline Tactical networks & $\begin{array}{l}\text { Military communications, operations } \\
\text { Automated Battlefields [1] }]\end{array}$ \\
\hline Sensor networks & $\begin{array}{l}\text { Collection of embedded sensor devices used to collect } \\
\text { real-time data to automate everyday functions [12]. }\end{array}$ \\
\hline Emergency services & Search-and-rescue operations as well as disaster recovery [13]. \\
\hline Commercial environments & $\begin{array}{l}\text { E-Commerce, e.g., electronic payments from anywhere } \\
\text { Local ad-hoc network with nearby vehicles for } \\
\text { road/ accident guidance [14] }\end{array}$ \\
\hline Educational applications & $\begin{array}{l}\text { Home/ office wireless networking (WLAN), } \\
\text { Personal area network (PAN) }\end{array}$ \\
\hline Location-aware services & $\begin{array}{l}\text { Set up virtual classrooms or conference rooms } \\
\text { meetings, or lectures [15] }\end{array}$ \\
\hline Infortainment & $\begin{array}{l}\text { Multiuser games } \\
\text { Robotic pets [16] } \\
\text { Outdoor Internet access }\end{array}$ \\
\hline
\end{tabular}

network cannot be trusted for the correct execution of critical network functions. This is one of the key issues which can lead to the security problems in mobile ad-hoc networks. 


\subsubsection{Characteristics of Mobile Ad-hoc Networks}

Unlike the networks using dedicated nodes to support basic functions like packet forwarding, routing and network management, ad-hoc networks carry out those functions by all available nodes. Several characteristics of mobile ad-hoc networks are presented below: $[9,17]$.

\section{Mobility}

The nodes in mobile ad-hoc networks can be rapidly repositioned or can move freely in an area. This large degree of freedom makes mobile ad-hoc networks completely different from any other networking solution, since the topology may be changing quickly and dynamically.

\section{Multihop routing}

In mobile ad-hoc networks, every node can act as a router and forward each other's packets to enable information sharing. Since there are obstacle negotiation and energy conservation issues, the data transmission from the resource to the destination needs multiple hops. For example, in the battle field, a sequence of short hops can reduce the probability of being detected by the enemy in the covert operations.

\section{Self-organization}

In ad-hoc networks, each node has self-organizing capabilities and works in a distributed peer-to-peer mode. It acts as an independent router and generates independent data. Each node autonomously determines its own configuration parameter in ad-hoc networks, and MANET does not depend on any established infrastructure. 


\section{Energy Conservation}

The power supplies for the nodes in ad-hoc networks are always limited, such as laptops and sensors. Each node works with multi responsibilities, such as a router and data generator. It should autonomously configure its network address and have to resolve the way to reach a destination. So the energy efficiency is critical for the longevity of the mission.

\section{Scalability}

Based on the self-organizing capabilities, the scalability of ad-hoc network is very strong. In some applications, the number of the nodes in ad-hoc networks can grow to thousands of nodes.

\subsection{Security in Mobile Ad-hoc Networks}

Compared with the fixed-wire line networks, mobile ad-hoc networks are more vulnerable to information and physical security threats.

The unique characteristics of MANETs which we talked above present some new challenges to security design due to the lack of any central authority and shared wireless medium [2].

As a distributed non-infrastructure network, the network security mainly relies on the individual security solution of each mobile node in the MANET, since it is very difficult or it is impossible to implement the traditional centralized security control.

The key issues we considered for the security requirements in mobile ad-hoc networks include $[18,19]$ :

- Access control: to protect the access to the wireless network infrastructure [20].

- Confidentiality: to protect the passive eavesdropping. 
- Data integrity: to prevent the tampering with traffic [21].

There are various security threats that exist in MANETs, such as denial of service, black hole, resource consumption, location disclosure, wormhole, host impersonation, information disclosure, and interference $[3,4]$.

A number of researchers have investigated the security issues in MANETs. Basically, there are two complementary classes of approaches to secure MANETs: prevention-based approaches, such as authentication, and detection-based approaches, such as intrusion detection systems (IDSs) $[3,22,23]$.

As the first line of defense, user authentication is crucial for confidentiality, integrity, and non-repudiation. Serving as the second wall of protection, IDSs can effectively help identify malicious activities [24]. Authentication is an important type of responses initiated by an IDS. After the authentication process, only authenticated users can continue using the network resources while compromised users will be excluded [25].

Zhang and Lee in [26] not only presented the basic requirements for an IDS which works in the MANETs environment, but also proposed a general intrusion detection and response mechanism for MANETs. In their proposed scheme, each IDS agent is involved in the intrusion detection and response tasks independently.

\subsection{Security in Vehicular Ad-hoc Networks}

Vehicular Ad-hoc Networks (VANETs), as a specific case of ad-hoc networks, have attracted more and more researchers not only from academic but also from industry all over the world. Besides vehicle-to-infrastructure (V2I) communication, vehicleto-vehicle $(\mathrm{V} 2 \mathrm{~V})$ communication is a typical type of communication in VANETs. In V2V communication, moving vehicles can work as wireless routers and autonomously organize and communicate with each other to create a mobile network. It can be 


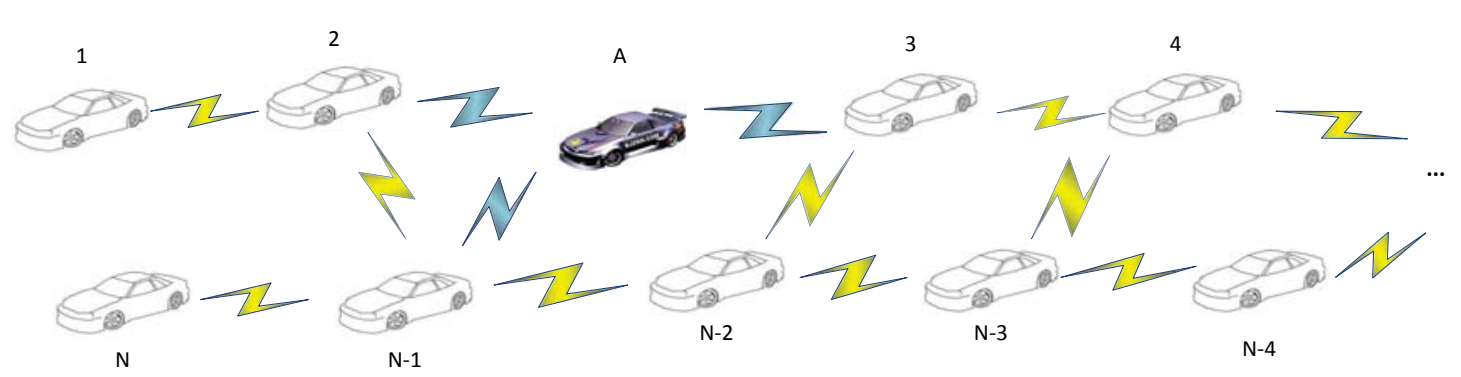

Figure 2: A $N+$ 1-node VANET with a malicious vehicle.

used to send emergency and real-time information such as an accident or road traffic information so that other vehicles can take alternative routes to prevent traffic congestions [10].

For these important applications, security becomes one of the key issues in VANETs. The security requirements such as privacy, integrity, and confidentiality to provide secured communications against attackers need to be considered and followed. If the emergency information is fake or modified by the attacker, the whole traffic may be influenced simultaneously. Meanwhile, the privacy of drivers and passengers also needs to be protected.

As shown in Fig. 2, the attacker vehicle in VANETs has been classified as three dimensions: insiders vs outsider, malicious vs rational and active vs passive [10,27]. Many kinds of attacks such as Sybin attack [28, 29], Denial of Service (DoS) [30], Wormhole attack [31], Illusion attack [32], and Purposeful attack [33] have been found in VANETs. A kind of attack named timing attack is introduced in [27]. When the emergency message is received by a malicious vehicle, it adds some spiteful delay to the original message instead of forwarding it to the neighboring vehicles at the right time. In the communication of VANETs, each kind of those attacks can affect the traffic safety deeply.

Recently, many researchers have proposed game theoretic approaches to improve network security $[34,35]$, since game theory can be used as a useful tool to provide 
a mathematical framework for modeling and analyzing decision problems. It can address problems where multiple players with contradictory goals or incentives compete with each other. In game theory, one player's outcome depends on his/her own decision and the other players' decisions. Similarly, the success of a security scheme in VANETs depends not only on the actual defense strategies, but also on the actions taken by the attackers.

Some researchers have tried to use game theory to solve the security problem in VANETs $[36,37]$. However, most of the existing work only considered a security game model with two players in the security game model: an attacker and a defender.

\subsection{Security in Cognitive Radio Mobile Ad-hoc Networks}

Cognitive radio (CR) [38] is an opportunistic communication technology which is designed to improve the utilization of the available licensed bandwidth for unlicensed users. With the cognitive radio technology, network users can make smart decisions on the usage of spectrum and operating parameters based on the sensed spectrum dynamics and actions adopted by other users. In CR-MANETs, mobile nodes cannot only autonomously organize and communicate with each other over bandwidthconstrained wireless links, but also have the capacity to sense the spectrum and choose the proper channels for data transmission.

However, due to the lack of any central authority and shared wireless medium, the challenges to security design in CR-MANETs are presented. These security problems, such as denial of service, black hole, resource consumption, information disclosure, and interference, are similar to those in MANETs. 
In [39], the researchers introduced two kinds of attacks from the exogenous attackers. One is the Jamming attack, and the other is the Incumbent Emulation attack. When an attacker floods the sensed channel with white/colored noise, energy detection for spectrum sensing can be used to specify this kind of jamming attack.

In [40], the authors extended the mean field game framework to a hierarchical interacting system for the cognitive wireless networks, which consisted of a finite number of primary users (PUs) and a large number of secondary users (SUs).

B. Wang et al. introduced an anti-jamming stochastic game for cognitive radio networks. The anti-jamming defense was proposed using the minimax-Q learning algorithm. The performance of using the optimal stationary policy was improved significantly [41].

There are three kinds of methods to distinguish the primary users and jamming attack, which are widely used for the spectrum sensing [42, 43].

- Energy detection: easy to implement and suboptimal method; fewer requirements on the position of PUs.

- Matched filter: much more complex and optimal method; more requirements to develop adaptive sensing circuits for different primary wireless systems.

- Cyclostationary feature detection: can detect the signals with very low SNR; require some prior knowledge of PUs.

For the radio jamming attack, its aim is to disrupt the communications at different layers in wireless networks, such as the physical layer and the link layer [41].

Usually, there are two types of jamming attacks. One type of jamming attack is to keep the wireless spectrum busy, so the legitimate users are prevented from accessing the open spectrum. The other is to let the SNR deteriorate by transmitting packets around the neighbors of the "sufferer". 
In [39], the authors discussed an exogenous attacker which can cause CRN service disruption through emitting jamming signals geared toward sensors, control channels, or receivers. The interference-resilient communications schemes which can decode the received signals in very low SNR regimes are needed.

\subsection{Game Theoretic Approaches for Security in MANETs}

Recently, game theoretic approaches have been recognized and proposed to improve network security not only in wired networks, but also in wireless networks $[34,35]$.

As a powerful tool to provide a mathematical framework for modeling and analyzing decision problems, game theory can address problems where multiple players with contradictory goals or incentives compete with each other. The history of game theory can date back to 1944 when J. Von Neumann and O. Morgenstern wrote and published the book "Theory of Games and Economic Behavior".

Game theory has been used primarily in economics for modeling the competition between companies. For instance, should a given company enter a new market or not? Then game theory has also been applied to other areas, including politics and biology.

John Von Neumann and Oskar Morgenstern wrote the first textbook in this area [44]. A few years later, John Nash made a number of additional contributions, the cornerstone of which was the famous Nash equilibrium [45]. Since then, many other researchers have contributed to the development of game theory [46].

During the late 1940s, cooperative game theory came out and was used to analyze optimal strategies for different groups of individuals. In 1950s, researchers developed lots of important concepts, such as the repeated games and the Shapley value. 
In the 1960s, Bayesian games and refinement of Nash equilibria were proposed.

J. M. Smith proposed the evolutionary game theory in the 1970s, which started the application of game theory in biology.

With the development of wired and wireless networks, game theory has also been realized and applied to solve the problems concerning resource allocation and routing in a competitive phenomenon [47].

In the past few years, researchers applied game theory to wireless communication networks. Players in the game have to cope with limited system resources, such as constrained battery life and limited computing capacity, which imposes conflict of interests that each player in the game tries to maximize its utility with minimum cost [48].

In this thesis, we assume that all the players in the game are rational. So all of players try their best to maximize their utility.

Since most interactions in wireless communication networks could be captured by using the concept of rationality with appropriate adjustment of the cost function, this assumption on the rationality of players is reasonable. For this purpose, the players react according to their competitors' strategies to minimize their cost [49].

In the view of game theory, one player's outcome depends not only on his/her own decision, but also on others' decisions. Similarly, the success of a security scheme in MANETs depends not only on the actual defense strategies, but also on the actions taken by the attackers [50].

Bedi et al. modeled the interaction between the attacker and the defender as a static game in two attack scenarios: one attacker for DoS and multiple attackers for DDoS [51]. The concept of multi-stage dynamic non-cooperative game with incomplete information was presented in [52], where an individual node with IDS can detect the attack with a probability depending on its belief updated according to 
its received messages. In [53], the authors integrated the ad-hoc on-demand distance vector (AODV) routing protocol for MANETs with the game theoretic approach. The benefit is that each node can transfer its packets through the route with less energy consumption of host-IDS and lower probability of attack with the optimal decision.

A framework that combines the N-intertwined epidemic model with noncooperative game model was proposed in [54], where the authors showed that the network's quality largely depends on the underlying topology. Researchers also tried to build an IDS based on a cooperative scheme to detect intrusions in MANETs $[55,56]$. The authors of $[57,58]$ considered a Bayesian game to study the interaction between the legitimate nodes and the malicious nodes. The malicious nodes try to deceive the legitimate nodes by cooperating with them to get better payoffs, and the legitimate nodes choose a probability to cooperate with the malicious nodes and decide whether or not to report misbehaviors based on their consistently updated beliefs.

Although some excellent research has been done on addressing the security issues in MANETs using game theoretic approaches, most of the existing work only considered a security game model with two players: an attacker and a defender. For the problem scenarios with multiple attackers versus multiple defenders, the security game is usually modeled as a two-player game in which the whole of the defenders is treated as one player, as is the whole of attackers [35]. While this assumption may be valid for a network with centralized administration, it is not realistic in MANETs, where centralized administration is not available. Consequently, each individual node in a MANET should be treated separately in the security game model.

In this study, using recent advances in mean field game theory [59], we propose a novel game theoretic approach for security in MANETs. The mean field game theory provides a powerful mathematical tool for problems with a large number of players. It 
has been successfully used by economists, socialists, and engineers in different areas, among others $[60,61]$.

\subsubsection{Mean Field Game}

Although the mean field game theory has came out as early as 2006 [62] and attracts lots of researchers, there are not so many papers studied on the security problem on the wireless networks.

Mean field game theory is devoted to analyze the games with a large number of "small" players. Here, the "small" means that the player has little influence on the overall system. However, since the number of the players is very large, these players can affect the system with their combined efforts [63, 64].

Jovanovic et al. firstly considered the strategic decision making problems in a very large populations of small interacting individuals in the economics literature in 1988.

In 2006, in the engineering literature, Huang et al. considered the stochastic dynamic games in large population conditions where multi-class agents are weakly coupled via their individual dynamics and costs. The idea is coming from the key common features that while each agent only receives a negligible influence from any other given individual, the effect of the overall population is significant to each other. Around the same time Lasry et al. did the similar work independently.

Recently, Huang et al. [59] developed a mixed mode of mean field game, which considered the situation where there are a major player and a large number of minor players. The players have decoupled state transition laws and are coupled by the costs via the state distribution of the minor players. A stochastic difference equation is introduced to model the update of the limiting state distribution process. With this method, the decision problems for the major player and minor players can be solved 
using the local information. A solvability assumption of the consistent mean field approximation is also introduced to obtain the stationary decentralized strategies for both the major player and minor players. This kind of model is very proper to be used to consider and analyze the security problem in MANETs.

There are three components in a game: set of players, set of players' strategies and set of players' utility functions.

- In the proposed game theoretic approach for security and precious system resources in mobile ad-hoc networks, we assume that there are a large number of minor players and one major attacker.

- The attacker selects an attacking target from all the minor players and tries its best to compromise the target node, while the defender adopts a strategic approach on action selection. The set of players' strategies are composed by all players' pure strategies. In the proposed mean field game theoretic approach, we assume that all the minor players can become the attacker's targets, thus attacker's strategy set consists of strategies: Attack and Not attack; the defenders' strategy set consists of strategies: Defend and Not Defend.

- Set of players' functions are made of the cost functions for all game players, which takes the strategic actions of players as input. In this mean field game, the major player's cost function depends on the major player's state, action and the state distribution of the minor players. A minor player's cost function depends on not only this minor player's state and action, but also the major player's state and the state distribution of all the minor players. From the structure of these cost functions, we can find that the major player has a significant impact on the minor players. By contrast, each minor player has a negligible impact on another minor player or the major player. 
More details of our proposed mean field game theory, such as the definitions of the states, actions, cost functions, translation laws and the approximation method for solving the problem will be introduced later.

\subsection{Summary}

In this chapter, first of all, fundamental concepts concerning mobile ad-hoc networks, including the developments and the characters, are presented. Secondly, the security issues that arise in mobile ad-hoc networks due to its decentralized organization and proposed approaches for combating security problems in mobile ad-hoc networks are discussed. Finally, application of game theoretic approach for security and source consumption is briefly described. In the next chapter, the proposed game theoretic approach for security and energy consumption in mobile ad-hoc networks is presented in details. 


\section{Chapter 3}

\section{Proposed Mean Field Game Theoretic}

\section{Approach}

In this chapter, we firstly describe the system which contains an $N$-node MANET and an attacker. Then the security problem of this system is formulated as an $N+1$ mean field game. The proposed game theoretic approach is described in details by setting up the system model and presenting the states, transition laws, and cost functions.

\subsection{System Description}

Fig. 3 illustrates an $N$-node MANET and an attacker that can attack the MANET dynamically. The legitimate nodes are independent because there is no centralized administration in the MANET. When the attacker has successfully attacked the MANET, some rewards (e.g., secret information) can be acquired by the attacker from the MANET. If the attacker failed because of the target node launching the defence action, some rewards (e.g., attack information) will be given to the target MANET node for its successful defence. Furthermore, the attacker and the defenders all need to pay the cost (e.g., energy consumption) for their individual actions. 


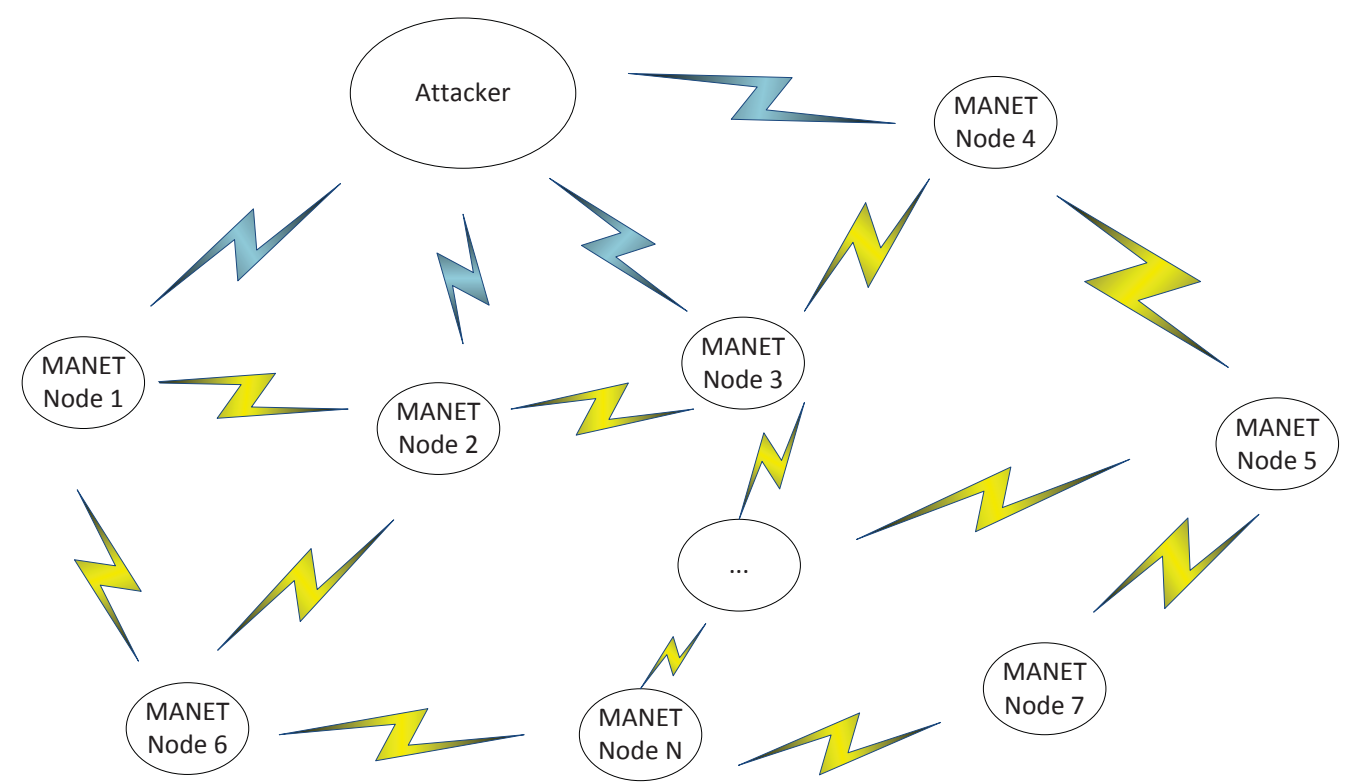

Figure 3: A N-node MANET with an attacker.

\subsection{Model Description}

\subsubsection{States, Transition Laws, and Cost Functions}

In this thesis, we model this system as an $N+1$ mean field game model. As shown in Fig. 4, there are $N$ minor players and one major player in this model. We consider the MANET nodes which can load the defending actions as the $N$ minor players. Meanwhile, the attacker, which tries to attack the MANET and influence the communication between the nodes, is considered as the major player $\mathcal{A}_{0}$. Fig. 4 illustrates the interactions between the major player and the minor players in the MANET. The major player makes decision based its own state $x_{0}$ to choose its action $u_{0}$. This action could be choosing which minor players as the targets to attack. So it can influence the whole MANET through the targeted nodes. Each node in this situation, could detect whether it has been attacked by the equipped IDS. The independent action $u_{i}$ is chosen by the minor player $\mathcal{A}_{i}$, which makes the decision based on not only its own state $x_{i}$ and the policy, but also the major player's action. We define $\rho_{i}$ and 


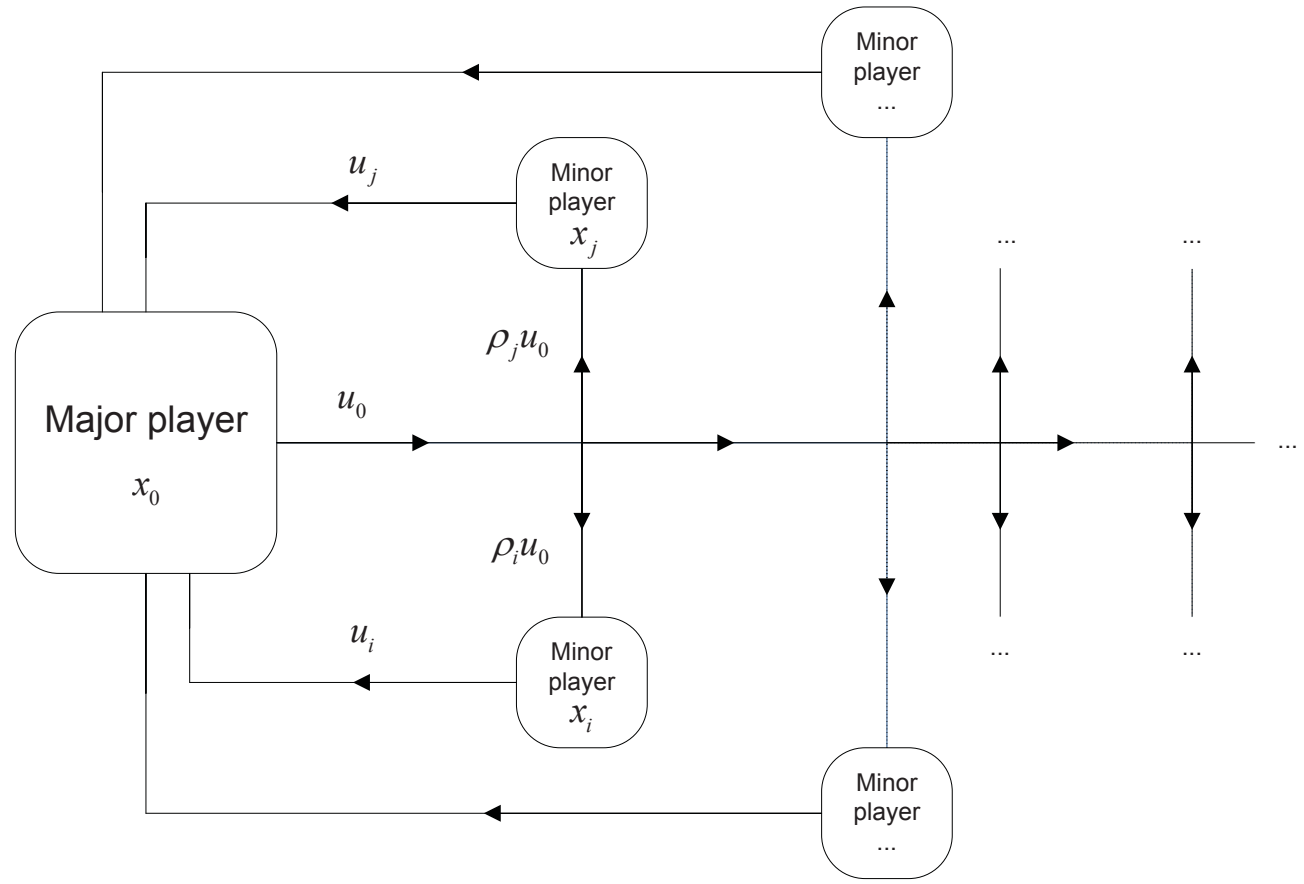

Figure 4: The mean field game model of a MANET with mixed players. $\left(x_{0}\right.$ : state of major player; $x_{i}, x_{j}$ : states of minor players $i$ and $j ; u_{0}$ : action of major player; $u_{i}$ and $u_{j}$ : actions of minor player $i$ and $j ; \rho_{i}$ and $\rho_{j}$ : weights of major player's action.) 
$\rho_{j}$ as the weights of the major player's action. These weights demonstrate that the attacker has different influence levels to the minor players in the same MANET. Since the distances may be different among the minor players to the major player and the direction of the major player's antenna could also lead to different levels of influence.

We define the attacker's state space and action space as $S_{0}=\left\{1, \cdots, K_{0}\right\}$ and $A_{0}=\left\{1, \cdots, L_{0}\right\}$, respectively. Meanwhile, the defenders' state space and action space are $S=\{1, \cdots, K\}$ and $A=\{1, \cdots, L\}$, respectively. At time $t \in \mathbb{Z}_{+}=\{0,1,2, \cdots\}$, we define that the attacker $\mathcal{A}_{0}$ 's state is $x_{0}(t)$ and its action is $u_{0}(t)$. Similarly, the state and the action of a representative legitimate node $\mathcal{A}_{i}, i \in(1, \cdots, N)$ are denoted as $x_{i}(t)$ and $u_{i}(t)$, respectively.

The major player's state is defined as a combination of energy and information assets, which can be denoted by $\alpha_{\mathrm{E}_{0}} E_{0}+\alpha_{I} I_{0}$ [65], in which $\alpha_{\mathrm{E}_{0}}$ and $\alpha_{I}$ represent the weights of energy and the information assets, respectively. Meanwhile, the minor players' state is defined as a combination of energy and security assets, which is denoted by $\alpha_{\mathrm{E}_{i}} E_{i}+\alpha_{S} S_{i}$, in which $\alpha_{\mathrm{E}_{i}}$ and $\alpha_{S}$ represent the weights of the energy and the security assets, respectively.

The average state of all the minor players is denoted by $I^{(N)}(t)$ and

$$
I^{(N)}(t)=\left(I_{1}^{(N)}(t), \cdots, I_{K}^{(N)}(t)\right),(t \geq 0)
$$

where $I_{K}^{(N)}(t)=\frac{1}{N} \sum_{i=1}^{N} 1_{\left(x_{i}(t)=K\right)} \cdot I^{(N)}(t)$ represents the frequency of occurrence of the states in $S$ in the mean field at time $t$.

Additionally, $Q_{0}\left(z \mid y, a_{0}\right)$ and $Q\left(z \mid y, a_{i}\right)$ represent the state transition laws of the major player and representative minor player, respectively. The state transition of the major player is specified by

$$
Q_{0}\left(z \mid y, a_{0}\right)=P\left(x_{0}(t+1)=z \mid x_{0}(t)=y, u_{0}(t)=a_{0}\right)
$$


where $y, z \in S_{0}$ and $a_{0} \in A_{0}$. For minor player $\mathcal{A}_{i}$, the state transition law is determined by

$$
Q(z \mid y, a)=P\left(x_{i}(t+1)=z \mid x_{i}(t)=y, u_{i}(t)=a\right)
$$

where $y, z \in S$, and $a \in A$.

The instantaneous costs of the major player and the representative minor player can be denoted by $c_{0}\left(x_{0}(t), u_{0}(t), I^{(N)}(t)\right)$ and $c_{i}\left(x_{i}(t), u_{i}(t), x_{0}(t), I^{(N)}(t)\right)$, respectively.

However, when we consider the game process, $c_{i}\left(x_{i}(t), u_{i}(t), u_{0}(t), I^{(N)}(t)\right)$ should be considered. Because it is believed that the impact of the major player to the representative minor player's instantaneous cost is not directly from the state $x_{0}(t)$, but directly from the action $u_{0}(t)$. In other words, for the representative minor player, at time $t$, the result of the game is not only determined by its action under certain state, but also depending on which action the major player takes under some state. We define the instantaneous cost of the major player as follows:

$$
\begin{aligned}
& c_{0}\left(x_{0}(t), u_{0}(t), I^{(N)}(t)\right) \\
& =f_{0}\left(x_{0}(t), u_{0}(t)\right)-f\left(I^{(N)}(t)\right),
\end{aligned}
$$

where $f_{0}\left(x_{0}(t), u_{0}(t)\right)$ denotes the coupled energy cost when the major player adopts different actions under various states. For example, when one state is "full energy" and the major player could choose the action to strongly attack the whole network. As a result, the energy cost is much higher than the one when the state is "poor energy" and the major player does not attack. $f\left(I^{(N)}(t)\right)$ denotes the payoff of the major player, which comes from the attacking. $f\left(I^{(N)}(t)\right)$ should also represent the average reflection of the whole mean field to the major player's attack. The instantaneous 
cost could be defined using this linear function.

Meanwhile, we also define the cost of a presentative minor player as follows:

$$
\begin{aligned}
& c_{i}\left(x_{i}(t), u_{i}(t), x_{0}(t), u_{0}(t), I^{(N)}(t)\right) \\
= & g_{i}\left(x_{i}(t), u_{i}(t)\right)-g_{0}^{i}\left(I^{(N)}(t), x_{0}(t), u_{0}(t)\right) .
\end{aligned}
$$

In the equation above, $g_{i}\left(x_{i}(t), u_{i}(t)\right)$ denotes the coupled cost when the presentative minor player adopts different actions under one state. $g_{0}^{i}\left(I^{(N)}(t), x_{0}(t), u_{0}(t)\right)$ represents the combined cost from the influence of the major player's state, action, and the reflection of the whole mean filed.

The interactions between the major player (as an attacker) and a representative minor player (as a defender) are modeled as a non-cooperative non-zero-sum game. We define that the minor player $\mathcal{A}_{i}^{\prime}$ security value is worth of $w_{i}$, where $w_{i}>0$. $w_{i}$ can be the value of the protected assets in practice and $-w_{i}$ represents a loss of security. In this model, we also assume that the loss $w_{i}$ of the minor player $\mathcal{A}_{i}$ is equal to the gain of the major player $\mathcal{A}_{0}$ from $\mathcal{A}_{i}$. However, the $\mathcal{A}_{0}$ could gain the $\sum_{i=1}^{N} w_{i}$ from different minor players at the same time. The game model of the ad-hoc network with a major player and several minor players is shown as Fig. 4.

\subsubsection{Mean Field Equation System}

By using the mean field approximation approach for overcoming the fundamental complexity, the mean field equation system can be given as follows [60]:

$$
\theta(t+1)=\varphi\left(x_{0}(t), \theta(t)\right)
$$




$$
\begin{aligned}
& v\left(x_{0}, \theta\right)=\min _{u_{0} \in A_{0}}\left\{c_{0}\left(x_{0}, u_{0}, \theta\right)+\Delta\right\}, \\
& w\left(x_{i}, x_{0}, \theta\right)=\min _{u_{i} \in A}\left\{c\left(x_{i}, u_{i}, x_{0}, \theta\right)+\Omega\right\},
\end{aligned}
$$

where

$$
\Delta=\rho \sum_{k \in S_{0}} Q_{0}\left(k \mid x_{0}, u_{0}\right) v\left(k, \varphi\left(x_{0}, \theta\right)\right),
$$

and

$$
\Omega=\rho \sum_{j \in S, k \in S_{0}} Q\left(j \mid x_{i}, u_{i}\right) Q_{0}\left(k \mid x_{0}, \hat{\pi}_{0}\right) w\left(j, k, \varphi\left(x_{0}, \theta\right)\right) .
$$

$\theta(t)$ presents a limiting process, which is used to approximate the random measure process $I^{(N)}(t)$ for a low complexity solution. We will discuss this more deeply in the next section. (7) and (8) are the dynamic programming equations for the major player and a representative minor player, respectively. Additionally, as we mentioned before, $c\left(x_{i}, u_{i}, x_{0}, \theta\right)$ is transformed from $c\left(x_{i}, u_{i}, x_{0}, u_{0}, \theta\right)$ in (8).

In this section, we first introduce the mean field approximation approach. Then the assumption of the $\varphi$ function and the formulation of the cost are presented. Finally, we discuss the solution to the mean field equation system. 


\subsection{Approximation of the Mean Field Process}

In MANETs, it is difficult to directly and promptly obtain $I^{(N)}(t)$, which represents the average state of all the minor players, due to the dynamic changing topology and the lack of centralization administration. To overcome the fundamental complexity, a method can be used to approximate the random measure process $I^{(N)}(t)$ with a limiting process $\theta(t)$. The updating rule of the limiting process $\theta(t)$ is proposed as (6), in which $\theta(0)=\theta_{0}$. In MANETs, this equation means that the random process's update is driven by the attacker's current state and the current average state of MANETs. Let $\mathcal{D}_{k}=\left\{\left(\lambda_{1}, \ldots, \lambda_{k}\right) \in \mathbb{R}_{+}^{k} \mid \sum_{j=1}^{k} \lambda_{j}=1\right\}, \varphi$ function could be considered from the following function class:

$$
\psi=\left\{\phi(i, \theta)=\left(\phi_{1}, \ldots, \phi_{k}\right) \mid \phi_{k} \geq 0, \sum_{k \in S} \phi_{k}=1\right\}
$$

where $\phi(i, \cdot)$ is continuous on $\mathcal{D}_{k}$ for all $i \in S_{0}$.

Considering the range of $I^{(N)}(t)$ is a discrete set, we take an approximation procedure for any $\theta \in \mathcal{D}_{k}$. The following key theorem is given on the asymptotic property of the update of $I^{(N)}(t)[60]$.

Theorem 1. Fix any $\theta=\left(\theta_{1}, \ldots, \theta_{K}\right) \in \mathcal{D}_{k}$. Suppose the major player (attacker) applies the optimal strategy $\hat{\pi}_{0}$ and the $N$ minor players (defenders) apply the optimal strategy $\hat{\pi}$, and at time $t$ the state of the major player is $x_{0}$ and $I^{(N)}(t)=\left(s_{1}, \ldots, s_{K}\right)$, where $\left(s_{1}, \ldots, s_{K}\right) \rightarrow \infty$, when $N \rightarrow \infty$. Then given $\left(x_{0}, I^{(N)}(t), \hat{\pi}\right)$, as $N \rightarrow \infty$,

$$
\begin{aligned}
I^{(N)}(t+1) \rightarrow & \left(\sum_{l=1}^{K} \theta_{l} Q\left(1 \mid l, \hat{\pi}\left(l, x_{0}, \theta\right)\right), \ldots,\right. \\
& \left.\sum_{l=1}^{K} \theta_{l} Q\left(K \mid l, \hat{\pi}\left(l, x_{0}, \theta\right)\right)\right)
\end{aligned}
$$


with probability one.

Proof. By the assumption on $I^{(N)}(t)$, there are $s_{k} N$ minor players in state $K \in$ $S$ at time $t$. In determining the distribution of $I^{(N)}(t+1)$, by symmetry of the minor players, we may assume without loss of generality that at time $t$ minor players $\mathcal{A}_{1}, \cdots, \mathcal{A}_{s_{1} N}$ are in state $1, \mathcal{A}_{s_{1} N+1}, \cdots, \mathcal{A}_{s_{1}+s_{2} N}$ are in state 2 , etc. We check the contribution of $\mathcal{A}_{1}$ alone in generating different states in $S$. Due to the transition of $\mathcal{A}_{1}$, state $K \in S$ will appear with probability

$$
Q\left(1 \mid l, \hat{\pi}\left(l, x_{0}, \theta\right)\right)
$$

We further obtain a probability vector

$$
Q 1:=\left(Q\left(k \mid 1, \hat{\pi}\left(1, x_{0}, \theta\right)\right)\right)_{k=1}^{K}
$$

with its entries assigned on the set $S$ indicating the probability that each state appears resulting from the transition of $\mathcal{A}_{1}$.

An important fact is that in the closed-loop system with $x_{0}(t)=x_{0}$, conditional independence holds for the transition from $x_{i}(t)$ to $x_{i}(t+1)$ for the $N$ processes.

Thus, the distribution of $N I^{N}(t+1)$ given $\left(x_{0}, I^{N}(t), \hat{\pi}\right)$ is obtained as the convolution of $N$ independent distributions corresponding to all $N$ minor players. And $Q_{1}$ is one of these $N$ distributions. We have

$$
\begin{aligned}
E_{x_{0}, I^{(N)}(t), \hat{\pi}} I^{(N)}(t+1)= & \left(\sum_{l=1}^{K} s_{l} Q\left(1 \mid l, \hat{\pi}\left(l, x_{0}, \theta\right)\right), \ldots,\right. \\
& \left.\sum_{l=1}^{K} s_{l} Q\left(K \mid l, \hat{\pi}\left(l, x_{0}, \theta\right)\right)\right),
\end{aligned}
$$

where $E_{x_{0}, I^{(N)}(t), \hat{\pi}}$ denotes the conditional mean given $\left(x_{0}, I^{N}(t), \hat{\pi}\right)$. 
So by the law of large numbers $I^{N}(t)-E_{x_{0}, I^{(N)}(t), \pi^{\prime}} I^{(N)}(t+1)$ converges to zero with probability one, as $N \rightarrow \infty$. We obtain (12).

Based on the right hand side of (12), we introduce the $K \times K$ matrix

$$
\begin{aligned}
& Q^{*}\left(x_{0}, \theta\right) \\
& =\left[\begin{array}{ccc}
Q\left(1 \mid 1, \hat{\pi}\left(1, x_{0}, \theta\right)\right) & \ldots & Q\left(K \mid 1, \hat{\pi}\left(2, x_{0}, \theta\right)\right) \\
Q\left(1 \mid 2, \hat{\pi}\left(2, x_{0}, \theta\right)\right) & \ldots & Q\left(K \mid 2, \hat{\pi}\left(2, x_{0}, \theta\right)\right) \\
\vdots & \ddots & \vdots \\
Q\left(1 \mid K, \hat{\pi}\left(K, x_{0}, \theta\right)\right) & \ldots & Q\left(K \mid K, \hat{\pi}\left(K, x_{0}, \theta\right)\right)
\end{array}\right] .
\end{aligned}
$$

The function $\varphi$ can be revised with

$$
\varphi\left(x_{0}, \theta\right)=\theta Q^{*}\left(x_{0}, \theta\right) .
$$

\subsubsection{Error Estimate on the Mean Field Approximation}

The following error estimate on the mean field approximation is important for the examination of the system performance. Here we introduce the following theorem [60]:

Theorem 2. Suppose $\theta(t)$ is generated by $\theta(t+1)=\varphi\left(x_{0}(t), \theta(t)\right)$ and $\left(\hat{\pi_{0}}, \hat{\pi}, \varphi\left(x_{0}, \theta\right)\right)$ is a consistent solution to the mean field equation system (6)-(8) and (17). We have

$$
\lim _{N \rightarrow \infty} E\left|I^{(N)}(t)-\theta(t)\right|=0
$$

for each given $t$.

Proof. We use the technique introduced in the proof of Theorem 1. Fix any $\epsilon \geq 0$. 
We have

$$
P\left(\left|I^{(N)}(0)-\theta_{0}\right| \geq \epsilon\right) \leq E\left|I^{(N)}(0)-\theta_{0}\right| / \epsilon \text {. }
$$

We take a sufficiently large $N_{0}$ such that for all $N \geq N_{0}$, we have

$$
P\left(\left|I^{(N)}(0)-\theta_{0}\right| \geq \epsilon\right)>1-\epsilon .
$$

Then following the method for (15), we may estimate $I^{(N)}(1)$. By the consistency condition (17), we further obtain

$$
\lim _{N \rightarrow \infty} E\left|I^{(N)}(t)-\theta(t)\right|=0
$$

Carrying out the estimates recursively, we obtain the desired result for each fixed $t$.

Based on Theorem 2 the approximation limit process $\theta$ could be used to instead of $I^{(N)}(t)$. We have supposed that the defenders' state space is $S=\{1, \cdots, K\}$, so the limiting process $\theta(t)$ in (6) should contain $K$ vectors: $\theta(t)=\left\{\theta_{1}(t), \cdots, \theta_{K}(t)\right\}$, where $\theta_{1}(t)+\cdots+\theta_{K}(t)=1$. In the mean field, when the major player is under different states, the influence to the whole mean field is different. Similarly, we consider that the state of the attacker drives the evolution of $\theta(t)$ in the MANET.

For ease of presentation, we assume the minor player has two states. The limiting process $\theta(t)$ should contain two vectors: $\theta(t)=\left\{\theta_{0}(t), \theta_{1}(t)\right\}$, where $\left(\theta_{0}(t)+\theta_{1}(t)=1\right)$. Here, $\theta_{0}(t)$ indicates the probability of the minor players' state $x_{i}=0$ and $\theta_{1}(t)$ indicates the probability of the minor players' state $x_{i}=1$. 
We assume the updating rule of $\theta_{0}$ is

$$
\varphi=x_{0}\left(\theta_{0}\right)^{1 / 2}+\left(1-x_{0}\right)\left(\theta_{0}\right)^{2}
$$

where $\theta_{0} \in[0,1]$. When the major player has chosen a state $x_{0}$ from $\{0,1\}$, the function $\varphi$ will be transformed to

$$
\varphi=\left\{\begin{array}{c}
\left(\theta_{0}\right)^{2},\left(x_{0}=0\right) \\
\left(\theta_{0}\right)^{1 / 2},\left(x_{0}=1\right)
\end{array}\right.
$$

The assumed function indicates that when the major player's state is $x_{0}=0$, it has a much greater influence to the mean field, because it has a larger derivative. We use (22) as the defined updating rule, since it has the following three strengths. Firstly, it clearly indicates that when the attacker chooses two different actions, the tendencies of the mean state of the whole MANET are almost opposite. Secondly, no matter which kind of actions is chosen, the update rule always obeys the asymptotic property. Thirdly, the range of the updating rule is from zero to one as well. So we use this definition of updating rule in our research.

\subsubsection{Cost Functions with the Approximation of Mean Field Process}

We let $\gamma_{i} \alpha_{i}-\left(1-\gamma_{i}\right) \beta_{i}$ represent the value of security protected by the representative minor player and $\left(1-\gamma_{i}\right) \beta_{i}$ denote the reward the attacker gets from the representative minor player for its attacking action. The attacker may attack several nodes at

the same time, so the reward of $\mathcal{A}_{0}$ should be $\sum_{i=1}^{N}\left(1-\gamma_{i}\right) \beta_{i}$. Here $\gamma_{i} \in[0,1]$ denotes the successful defending rate of the representative minor player and $\left(1-\gamma_{i}\right)$ denotes 
the unsuccessful defending rate. $\alpha_{i}$ is defined as the security value of the minor player $\mathcal{A}_{i}$ with successfully defending, and $\beta_{i}$ represents the loss of the security value with unsuccessfully defending, where $\alpha_{i}, \beta_{i}>0$.

Considering the average effect of the mean field to the major player and the representative minor player, we get the new cost functions as follows:

$$
\begin{aligned}
& c_{0}\left(x_{0}(t), u_{0}(t), \theta(t)\right) \\
= & f_{0}\left(x_{0}(t), u_{0}(t)\right)-\theta(t) \sum_{i=1}^{N}\left(1-\gamma_{i}\right) \beta_{i}, \\
& c_{i}\left(x_{i}(t), u_{i}(t), x_{0}(t), u_{0}(t), \theta(t)\right) \\
= & g_{i}\left(x_{i}(t), u_{i}(t)\right)-\theta(t)\left[\gamma_{i} \alpha_{i}-\left(1-\gamma_{i}\right) \beta_{i}\right] .
\end{aligned}
$$

\subsubsection{Solution to Mean Field Equation System}

For solving the mean field equation system, we use the dynamic programming approach. By breaking complicated problems down into simpler subproblems, dynamic programming is widely used in solving these complex problems. Here, using the dynamic programming method, we can obtain the major player's optimal policy $\pi_{0}$ by the dynamic programming $(7)$. With the obtained $\pi_{0}$, the representative minor player's optimal policy $\pi$ can also be acquired by (8). Then, we revise the function $\varphi$ with (17).

Using the dynamic programming (7) with (24), we can get the major player's strategy

$$
\pi_{0}=\left(\alpha_{1}, \alpha_{2}, \cdots, \alpha_{L_{0}}\right)
$$

which is a probability vector. In each step of the game, using strategy $\pi_{0}$, the major player selects an action $K$ with probability $\alpha_{K}$. The new state transition law with 
optimal strategy

$$
Q_{0}\left(z \mid x_{0}, \pi_{0}\right)=\sum_{x_{0} \in S_{0}, u_{0} \in A_{0}} \alpha\left(u_{0} \mid x_{0}\right) Q_{0}\left(z \mid x_{0}, u_{0}\right)
$$

can be pursued. With this major player's new state transition law, the minor player's strategy can be obtained from (8) and (25). Similarly, the minor players' strategy

$$
\pi=\left(\beta_{1}, \beta_{2}, \cdots, \beta_{L}\right)
$$

and the new state transition law with optimal policy

$$
Q\left(z \mid x_{0}, \pi\right)=\sum_{x_{i} \in S, u_{i} \in A} \beta\left(u_{i} \mid x_{i}\right) Q\left(z \mid x_{i}, u_{i}\right)
$$

can be acquired by the similar method.

Finally, the function $\varphi$ can be revised with (17).

\subsection{Summary}

In this chapter, the system which contains an $N$-node MANET and an attacker was described. Then we formulate the security problem of this system as an $N+1$ mean

field game. The details of the proposed game theoretic approach is described by setting up the system model and presenting the states, transition laws, and cost functions. For overcoming the fundamental complexity, an approximation method is proposed in which a limiting process $\theta(t)$ to is used to approximate the random measure process $I^{(N)}(t)$. The defined updating rule we designed for $\theta(t)$ has three strengths. Firstly, it clearly indicates that when the attacker chooses two different actions, the tendencies of the mean state of the whole MANET are almost opposite. 
Secondly, no matter which kind of actions is chosen, the update rule always obeys the asymptotic property. Thirdly, the range of the updating rule is from zero to one as well.

In the next chapter, simulation results and discussion are presented to show the effectiveness of the proposed game theoretic approach for security and energy consumption in cooperative wireless communication networks. The system performance analysis is also presented in Chapter 4. 


\section{Chapter 4}

\section{MANETs Simulation Results and Discussions}

In this chapter, we evaluate the performance of the proposed game theoretic approach for security and energy consumption in mobile ad-hoc networks through extensive simulations using MATLAB. All simulations are executed on a laptop featured with Windows 8, Intel I5-3230M 2.6 GHz CPU, 6 GB memory and MATLAB R2012b.

We consider the following simulation scenarios: A MANET consists of $N$ nodes (such as $N=40$ ), each of which is equipped with IDS sensors. There is a malicious node which is considered as the major player. The $N$ nodes in the MANET are the minor players and they can detect the intrusion with the help of IDS sensors independently [66].

\subsection{Distributed Optimal Defending Strategy in MANETs}

Based on the above mean field game modeling and analysis, we use an example to show the stochastic distributed optimal defending strategy in MANETs in this 
section. The setups of the elements for both the major player and the representative minor player in the MANET is summarized in Table 2.

Table 2: Setups of the Elements for Both the Major Player and the Representative Minor Player in MANETs

\begin{tabular}{|c|c|c|c|c|c|c|c|}
\hline & \multicolumn{7}{|c|}{ Setups } \\
\hline $\begin{array}{l}\text { major player's state s- } \\
\text { pace }\end{array}$ & \multicolumn{7}{|c|}{$S_{0}=\{0,1\}$} \\
\hline $\begin{array}{l}\text { major player's action s- } \\
\text { pace }\end{array}$ & \multicolumn{7}{|c|}{$A_{0}=\{0,1\}$} \\
\hline $\begin{array}{l}\text { major player's state } \\
\text { transition matrices }\end{array}$ & \multicolumn{2}{|c|}{$Q_{0}\left(z \mid y, u_{0}=0\right)=$} & $\begin{array}{cc}0.7 & 0.3 \\
0.02 & 0.98\end{array}$ & \multicolumn{2}{|c|}{$Q_{0}\left(z \mid y, u_{0}=1\right)=$} & 0.9 & $\begin{array}{c}0.1 \\
0.99\end{array}$ \\
\hline $\begin{array}{l}\text { major player's cost } \\
\text { function }\end{array}$ & \multicolumn{7}{|c|}{$c_{0}\left(s_{0}, u_{0}, \theta\right)=f_{0}\left(s_{0}, u_{0}\right)-\theta_{0} \sum_{i=1}^{N}(1-\gamma) \beta_{i}$} \\
\hline $\begin{array}{l}\text { major player's cost ma- } \\
\text { trix }\end{array}$ & \multicolumn{2}{|r|}{$R_{0}=$} & $\begin{array}{l}\left(s_{0}=0, u_{0}=\right. \\
\left(s_{0}=1, u_{0}=\right.\end{array}$ & $\begin{array}{ll}\text {, } \theta) & c_{0} \\
\text {, } \theta) & c_{0}\end{array}$ & $\begin{array}{l}0=0, u_{0}= \\
0=1, u_{0}=\end{array}$ & & \\
\hline $\begin{array}{l}\text { a representative minor } \\
\text { player's state space }\end{array}$ & \multicolumn{7}{|c|}{$S_{i}=\{0,1\}$} \\
\hline $\begin{array}{l}\text { a representative minor } \\
\text { player's action space }\end{array}$ & \multicolumn{7}{|c|}{$A_{i}=\{0,1\}$} \\
\hline $\begin{array}{l}\text { a representative minor } \\
\text { player's state transition } \\
\text { matrix }\end{array}$ & \multicolumn{2}{|c|}{$Q\left(z \mid y, u_{i}=0\right)=$} & $\begin{array}{cc}0.8 & 0.2 \\
0.02 & 0.98\end{array}$ & \multicolumn{2}{|c|}{,$Q\left(z \mid y, u_{i}=1\right)=$} & $\begin{array}{c}0.9 \\
0.01\end{array}$ & $\begin{array}{c}0.1 \\
0.99\end{array}$ \\
\hline $\begin{array}{l}\text { a representative minor } \\
\text { player's cost function }\end{array}$ & \multicolumn{7}{|c|}{$c_{i}\left(s_{i}, u_{i}, u_{0}, \theta\right)=g_{i}\left(s_{i}, u_{i}\right)-\theta\left[\gamma_{i} \alpha_{i}-\left(1-\gamma_{i}\right) \beta_{i}\right]$} \\
\hline $\begin{array}{l}\text { a representative minor } \\
\text { plaver's cost matrices }\end{array}$ & $R_{1}=$ & $\begin{array}{l}c_{i}(0,0,0, \theta) \\
c_{i}(1,0,0, \theta)\end{array}$ & $\begin{array}{l}c_{i}(0,1,0, \theta) \\
c_{i}(1,1,0, \theta)\end{array}$ &,$R_{2}=$ & $\begin{array}{l}c_{i}(0,0,1, \\
c_{i}(1,0,1\end{array}$ & $\begin{array}{l}c_{i}(0 \\
c_{i}(1\end{array}$ & $\begin{array}{l}, 1,1, \theta) \\
, 1,1, \theta)\end{array}$ \\
\hline
\end{tabular}




\subsubsection{Mixed Strategies and State Transition Laws for the Major Player}

For ease of presentation, suppose that the major player's state space is $S_{0}=\{0,1\}$ and its action space is $A_{0}=\{0,1\}$. As we consider the state of the major player is a combination of energy and information assets, the state $x_{0}=0$ means the major player's state is positive aggression and it has full energy for getting more information. Similarly, $x_{0}=1$ denotes the negative aggression of the major player. $u_{0}=0$ means the major player takes the action "Attack", while $u_{0}=1$ denotes the action "Not Attack". Suppose the state transition matrices of the major player are

$$
\begin{aligned}
& Q_{0}\left(z \mid y, u_{0}=0\right)=\left[\begin{array}{cc}
0.7 & 0.3 \\
0.02 & 0.98
\end{array}\right], \\
& Q_{0}\left(z \mid y, u_{0}=1\right)=\left[\begin{array}{ll}
0.9 & 0.1 \\
0.01 & 0.99
\end{array}\right] .
\end{aligned}
$$

These matrices stand for the probabilities changing from one state to another state. For example, when major player's state is $x_{0}=0$, if it chooses action $u_{0}=0$, it can keep the state $x_{0}=0$ with probability 0.7 in next step and it can change to the state $x_{0}=1$ with probability 0.3 .

The setup of the above nodes' transition matrices and cost matrices below is a non-trivial task for the proposed scheme. In constructing these values, we assume that most node properties can be made known to the IDS, which should be realistic particularly for MANETs where initial planning and device management is an a priori requirement. By "node properties" we mean the states and information that are used as input to the transition and cost matrices. 
The cost function of the major player is defined as

$$
c_{0}\left(x_{0}, u_{0}, \theta\right)=f_{0}\left(x_{0}, u_{0}\right)-\theta_{0} \sum_{i=1}^{N}\left(1-\gamma_{i}\right) \beta_{i},
$$

where $f_{0}\left(x_{0}, u_{0}\right)=\left(2-x_{0}\right)\left(1-u_{0}\right), N=40$, the successful defending rate $\gamma_{i}=0.8$, and the loss of the security value with unsuccessfully defending $\beta_{i}=0.25$. Here, $f_{0}\left(x_{0}, u_{0}\right)=\left(2-x_{0}\right)\left(1-u_{0}\right)$ denotes the coupled cost when the major player $\mathcal{A}_{0}$ adopts different actions under various states. It is better for the major player $\mathcal{A}_{0}$ when $c_{0}\left(x_{0}, u_{0}, \theta_{0}\right)$ is much smaller. Because it means that the attacker can get more reward during its attack or it costs less for the attack. When $\theta_{0}$ tends to be 1 , it means most of the minor players are in positive defending states. If the major player chooses to attack under this situation, the successful defending rate $\gamma_{i}$ should be larger and the reward $\sum_{i=1}^{N}\left(1-\gamma_{i}\right) \beta_{i}$ should be smaller. So $c_{0}\left(x_{0}, u_{0}, \theta_{0}\right)$ should get a greater value. When $\theta_{0}$ is close to zero, it means most of the minor players are in negative defending states. Most of them may be compromised. Their successful defending rate should be lower and the major player can get much more rewards, so $c_{0}\left(x_{0}, u_{0}, \theta_{0}\right)$ should be a smaller value. As we define the cost matrix:

$$
R_{0}=\left[\begin{array}{cc}
c_{0}\left(x_{0}=0, u_{0}=0, \theta\right) & c_{0}\left(x_{0}=0, u_{0}=1, \theta\right) \\
c_{0}\left(x_{0}=1, u_{0}=0, \theta\right) & c_{0}\left(x_{0}=1, u_{0}=1, \theta\right)
\end{array}\right],
$$

we can get the cost function matrix:

$$
R_{0}=\left[\begin{array}{cc}
2-2 \theta_{0} & -2 \theta_{0} \\
1-2 \theta_{0} & 0
\end{array}\right]
$$

During the value iteration, we choose $\theta_{0}$ starting from 0.7 for the iteration. The 
update of $\theta_{0}$ and the minimum values of $v$ in each step are shown in Fig. 5.
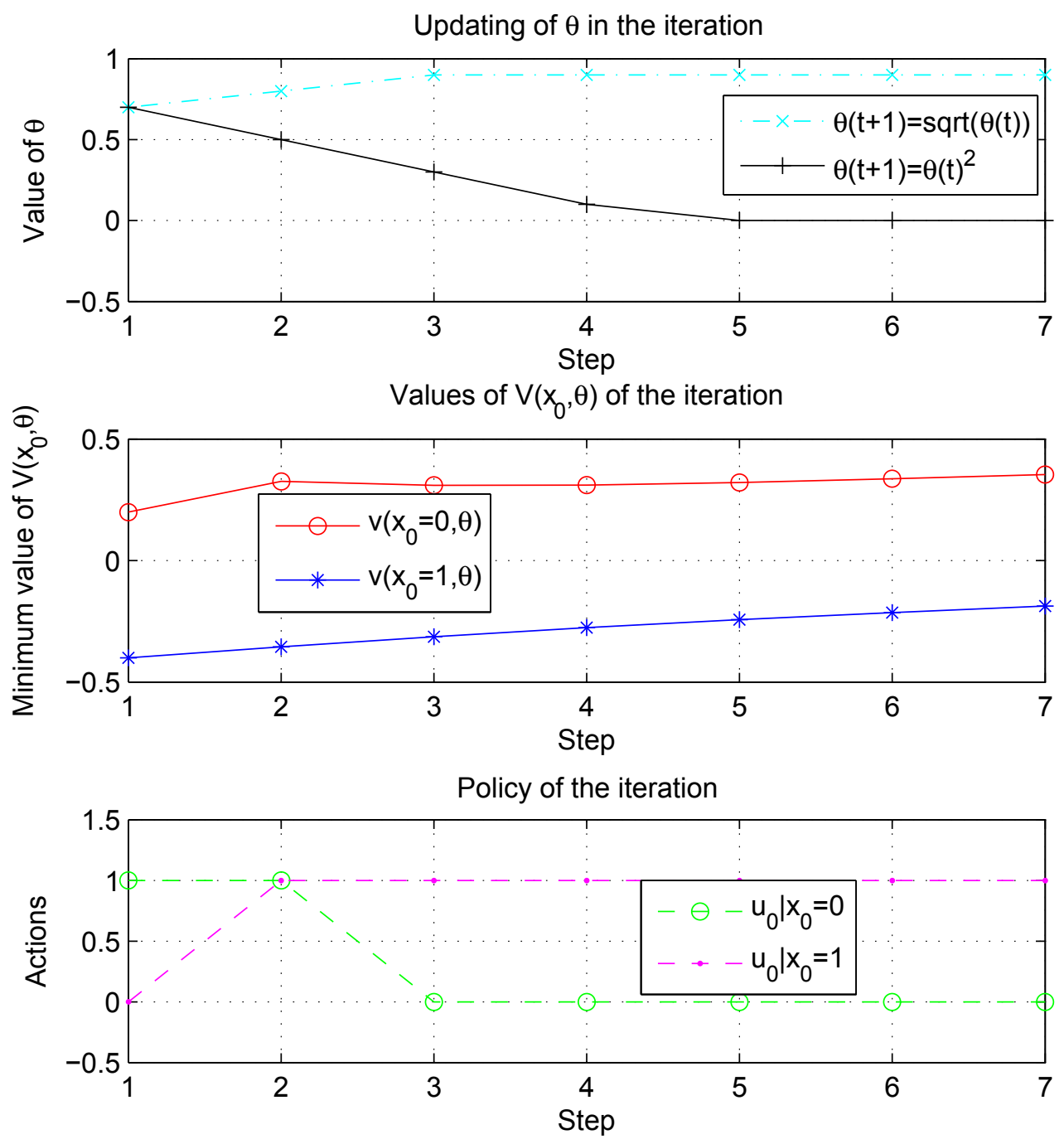

Figure 5: Value iteration for the major player.

We can see that, when the state of the major player is negative attacking, the values of $v$ are always below zero. The result also reflects that more attacks may not produce more rewards, if the defenders' successful detection rate is fixed. This is because the cost of attack may be much higher than the rewards for the major 
player. After the seventh step, the iteration stops and we can get the policy $\pi_{0}$ from the value iteration results:

$$
\pi_{0}^{0}=\left[\alpha\left(u_{0}=0 \mid x_{0}=0\right)=1, \alpha\left(u_{0}=1 \mid x_{0}=0\right)=0\right],
$$

when $x_{0}=0$, and

$$
\pi_{0}^{1}=\left[\alpha\left(u_{0}=0 \mid x_{0}=1\right)=0, \alpha\left(u_{0}=1 \mid x_{0}=1\right)=1\right]
$$

when $x_{0}=1$. Define

$$
Q_{0}\left(z \mid x_{0}, \pi_{0}\right)=\sum_{x_{0} \in S_{0}, u_{0} \in A_{0}} \alpha\left(u_{0} \mid x_{0}\right) Q_{0}\left(z \mid x_{0}, u_{0}\right)
$$

then we can get the matrix:

$$
Q_{0}=\left[\begin{array}{ll}
Q_{0}\left(0 \mid 0, \pi_{0}\right) & Q_{0}\left(1 \mid 0, \pi_{0}\right) \\
Q_{0}\left(0 \mid 1, \pi_{0}\right) & Q_{0}\left(1 \mid 1, \pi_{0}\right)
\end{array}\right]=\left[\begin{array}{cc}
0.7 & 0.3 \\
0.01 & 0.99
\end{array}\right] .
$$

\subsubsection{Mixed Strategies and State Transition Laws for a Rep- resentative Minor Player}

For the representative minor player we suppose the state space $S_{i}=\{0,1\}$ and action space $A_{i}=\{0,1\}$. Since the state of the representative minor player is a combination of energy asset and security asset, $x_{i}=0$ means the node is safe. It has full energy to do the positive defense and to keep the security value for itself. Meanwhile, $x_{i}=1$ denotes the node is unsafe and it can only do passive defense during the game process. $u_{i}=0$ means the Defend action and $u_{i}=1$ denotes the Not Defend action. We 
Table 3: Utility Matrix of the Representative Minor Player $\mathcal{A}_{i}$ in MANETs

\begin{tabular}{|c|c|c|}
\hline & Defend & Not defend \\
\hline Attack & $g_{i}\left(x_{i}, 0\right)-\theta_{d}\left[\gamma_{i} \alpha_{i}-\left(1-\gamma_{i}\right) \beta_{i}\right]$ & $g_{i}\left(x_{i}, 1\right)+\theta_{d} \beta_{i}$ \\
\hline Not attack & $g_{i}\left(x_{i}, 0\right)$ & 0 \\
\hline
\end{tabular}

set the representative minor player's state transition matrix as

$$
\begin{aligned}
& Q\left(z \mid y, u_{i}=0\right)=\left[\begin{array}{ll}
0.8 & 0.2 \\
0.02 & 0.98
\end{array}\right], \\
& Q\left(z \mid y, u_{i}=1\right)=\left[\begin{array}{ll}
0.9 & 0.1 \\
0.01 & 0.99
\end{array}\right] .
\end{aligned}
$$

The representative minor player's cost function is

$$
c_{i}\left(x_{i}, u_{i}, u_{0}, \theta\right)=g_{i}\left(x_{i}, u_{i}\right)-\theta\left[\gamma_{i} \alpha_{i}-\left(1-\gamma_{i}\right) \beta_{i}\right],
$$

where $g_{i}\left(x_{i}, u_{i}\right)=\left(1.8-x_{i}\right)\left(1-u_{i}\right), N=40, \gamma_{i}=0.8, \alpha_{i}=1$, and $\beta_{i}=1.5$.

Table 3 illustrates the cost matrix of the representative minor player $\mathcal{A}_{i}$ in the strategic form. As mentioned before, we transform $c_{i}\left(x_{i}, u_{i}, u_{0}, \theta\right)$ to the original definition $c_{i}\left(x_{i}, u_{i}, x_{0}, \theta\right)$. According to the optimal policy of the major player, the optimal action is corresponding to the state. When a state is determined, the optimal action is also determined with the optimal policy. So we can use $c_{i}\left(x_{i}, u_{i}, x_{0}, \theta\right)$ directly. 
The cost matrices of the representative minor player are

$$
R_{1}=\left[\begin{array}{cc}
c_{i}(0,0,0, \theta) & c_{i}(0,1,0, \theta) \\
c_{i}(1,0,0, \theta) & c_{i}(1,1,0, \theta)
\end{array}\right]
$$

and

$$
R_{2}=\left[\begin{array}{cc}
c_{i}(0,0,1, \theta) & c_{i}(0,1,1, \theta) \\
c_{i}(1,0,1, \theta) & c_{i}(1,1,1, \theta)
\end{array}\right]
$$

So we get

$$
R_{1}=\left[\begin{array}{cc}
1.8-0.3 \theta & 2.5 \theta \\
0.8-0.3 \theta & 2.5 \theta
\end{array}\right]
$$

and

$$
R_{2}=\left[\begin{array}{ll}
1.8 & 0 \\
0.8 & 0
\end{array}\right]
$$

To obtain the optimal policy of the representative minor player, we also choose $\theta_{0}$ starting from $\theta_{0}=0.7$ and updating with the same evolution rule. Fig. 6 illustrates the process of value iteration for a representative minor player. From the result of the iteration, we acquire the optimal action for the minor players. The optimal policy for the minor player is not only driven by the state of minor player, but also driven by the state of major player and $\theta$. 

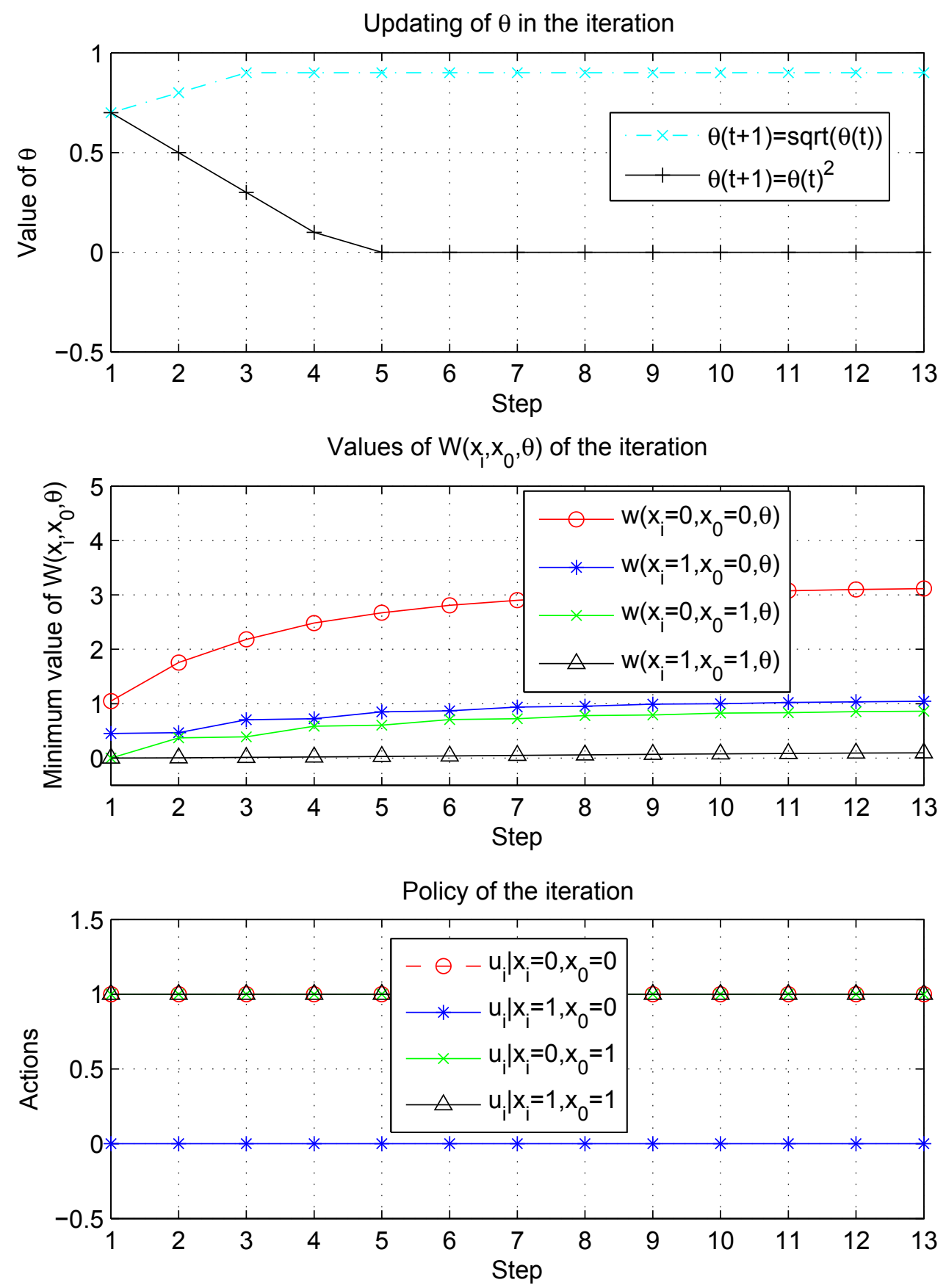

Figure 6: Value iteration for a representative minor player. 
The following matrices are the optimal policy $\pi$ from the value iteration:

$$
\begin{gathered}
\pi^{0}=\left[\begin{array}{l}
\beta\left(u_{i}=0 \mid x_{i}=0, x_{0}=0\right)=0, \\
\beta\left(u_{i}=1 \mid x_{i}=0, x_{0}=0\right)=1
\end{array}\right], \\
\pi^{1}=\left[\begin{array}{l}
\beta\left(u_{i}=0 \mid x_{i}=1, x_{0}=0\right)=1 \\
\beta\left(u_{i}=1 \mid x_{i}=1, x_{0}=0\right)=0
\end{array}\right], \\
\pi^{3}=\left[\begin{array}{l}
\beta\left(u_{i}=0 \mid x_{i}=0, x_{0}=1\right)=0 \\
\beta\left(u_{i}=1 \mid x_{i}=0, x_{0}=1\right)=1
\end{array}\right] \\
\pi^{4}=\left[\begin{array}{l}
\beta\left(u_{i}=0 \mid x_{i}=1, x_{0}=1\right)=0 \\
\beta\left(u_{i}=1 \mid x_{i}=1, x_{0}=1\right)=1
\end{array}\right]
\end{gathered}
$$

Define

$$
Q\left(z \mid x_{i}, \pi\right)=\sum_{x_{i} \in S, u_{i} \in A} \beta\left(u_{i} \mid x_{i}\right) Q\left(z \mid x_{i}, u_{i}\right),
$$

then we can get the state transition law of the representative minor player as follows:

$$
Q^{1}=\left[\begin{array}{cc}
Q(0 \mid 0, \pi) & Q(1 \mid 0, \pi) \\
Q(0 \mid 1, \pi) & Q(1 \mid 1, \pi)
\end{array}\right]=\left[\begin{array}{cc}
0.9 & 0.1 \\
0.02 & 0.98
\end{array}\right],
$$


when $x_{0}=0$ and

$$
Q^{2}=\left[\begin{array}{ll}
Q(0 \mid 0, \pi) & Q(1 \mid 0, \pi) \\
Q(0 \mid 1, \pi) & Q(1 \mid 1, \pi)
\end{array}\right]=\left[\begin{array}{cc}
0.9 & 0.1 \\
0.01 & 0.99
\end{array}\right]
$$

when $x_{0}=1$. The result illustrates that the optimal transition matrices are different for the representative minor player when the major player chooses different states. In a MANET, it may be hard to judge what state the attacker is, but it may be easy to detect the attacker's action. During the game process, we consider the attacker always runs with its optimal policy, so we can obtain the attacker's states by detecting its action here. These optimal state transition laws can work well for the minor player, no matter which action the major player chooses.

\subsubsection{Revising Function $\varphi$.}

As we mentioned before, we need to revise the function $\varphi$ after we get the optimal

policy of the representative minor player. Using the method in [60], we define the function $\varphi\left(x_{0}, \theta\right)=\theta Q^{*}\left(x_{0}, \theta\right)$, where $Q^{*}\left(x_{0}, \theta\right)$ is defined in (8). We get

$$
Q^{*}\left(x_{0}, \theta\right)=\left[\begin{array}{cc}
Q\left(0 \mid 0, \hat{\pi}\left(0, x_{0}, \theta\right)\right) & Q\left(1 \mid 0, \hat{\pi}\left(0, x_{0}, \theta\right)\right) \\
Q\left(0 \mid 1, \hat{\pi}\left(1, x_{0}, \theta\right)\right) & Q\left(1 \mid 1, \hat{\pi}\left(1, x_{0}, \theta\right)\right)
\end{array}\right]
$$

So the updating matrices of $\theta$ are

$$
Q^{*}\left(x_{0}=0, \theta\right)=\left[\begin{array}{cc}
0.9 & 0.1 \\
0.02 & 0.98
\end{array}\right]
$$


and

$$
Q^{*}\left(x_{0}=1, \theta\right)=\left[\begin{array}{cc}
0.9 & 0.1 \\
0.01 & 0.99
\end{array}\right] \text {. }
$$

\subsection{Simulation Results and Discussions}

\subsubsection{Simulation Scenarios}

In this section, we perform a numerical study on the following scenarios to validate our analytical results. We consider the following simulation scenarios: A MANET consists of $N$ nodes, each of which is equipped with IDS sensors. The number of nodes in the MANET will be changed in the simulations ( such as $N=20,40,60,80,100$ ). There is a malicious node that wants to attack this MANET. The malicious node is considered as the major player. The $N$ nodes in the MANET are the minor players and they can detect the intrusion with the help of IDS sensors operating independently.

We compare the performance of a representative node, which adopts the optimal strategy along with the performance of adopting other two strategies. The average lifetime and the compromising probability of the MANET are also compared. We also consider the situation of the nodes in the MANET with sufficient energy. The decrease of compromising probability and the improvement of lifetime using the optimal strategy are depicted.

First of all, the major player in the system starts with a dynamic state $x_{0} \in S_{0}$. It evolutes with its optimal updating rule and attacks the minor players randomly. The minor players' states start with safe and full energy and then they are updated with one of the two optimal updating rules, which are based on the influence of the major player. The minor players can detect the actions of the major player by the 
IDS sensors, which are equipped in each node.

\subsubsection{Average Cost}

Using the cost function defined in (41), we perform the simulation 200 times and calculate a representative minor player's the average cost of each step. We compare the average cost under different strategies, such as the security-prioritized strategy, the energy-prioritized strategy, and the optimal strategy. In the energy-prioritized strategy, energy is the primary concern of the mobile nodes (e.g., in commercial MANETs) [67], while security is the primary concern in the security-prioritized strategy (e.g., in military MANETs) [68].

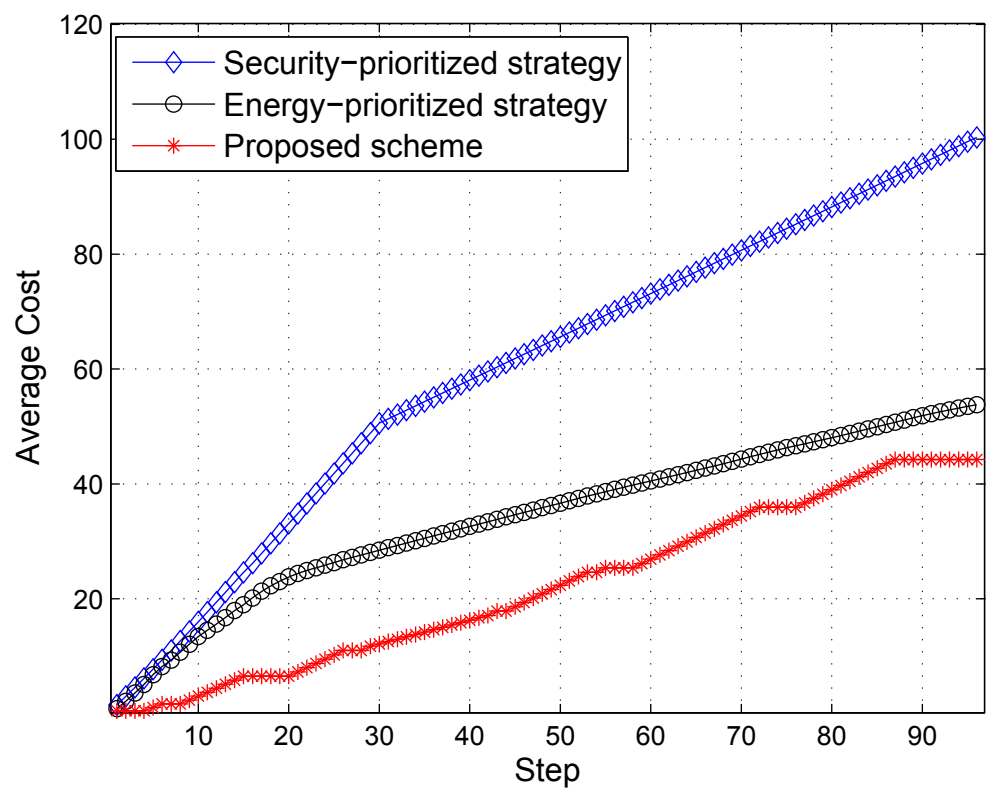

Figure 7: Average cost comparison among the representative minor player with security-prioritized strategy, energy-prioritized strategy and optimal strategy (under the dynamical attack).

From Fig. 7, we can see that the proposed scheme has the lowest cost compared with the other two strategies. In other words, each minor player can greatly reduce 


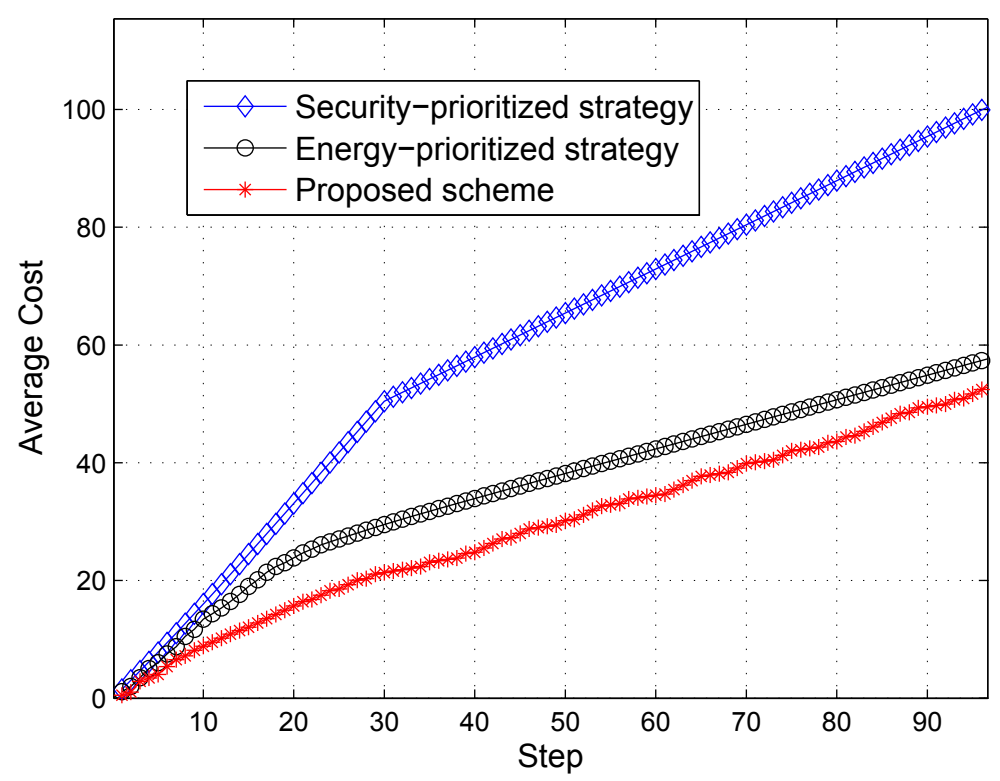

Figure 8: Average cost comparison among the representative minor player with security-prioritized strategy, with energy-prioritized strategy and with optimal strategy (under the continuous attack).

energy consumption and loss of security information with the optimal strategy. When the major player attacks continuously, as illustrated in Fig. 8, the average cost when the minor player adopts the optimal strategy is also the lowest compared with the other two strategies.

\subsubsection{Defence Actions According to Optimal Strategy}

In the simulation, we study minor players' defence actions when the attacker launches continuous attack and dynamic attack, respectively. We choose a MANET with 20 nodes $(N=20)$ and capture nodes' actions in each step. All the MANET nodes use the optimal strategy derived in our scheme.

In Fig. 9, the attacker launches continuous attack and it chooses attacking target dynamically. We records and observes the dynamic change of the target node's action with the proposed scheme in the first 50 steps. From the simulation result, we can see 


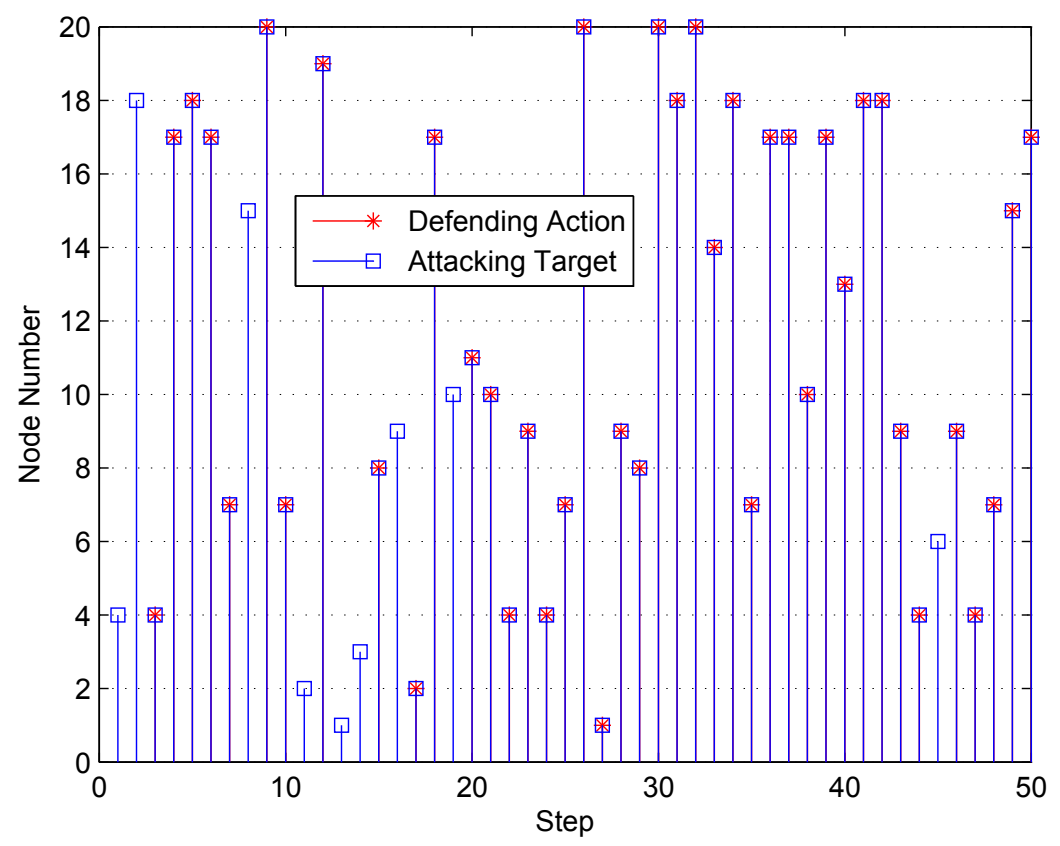

Figure 9: Attacking target and defence action under major player's continuous attack.

that the defender being attacked in each step does not always choose the defending action with the optimal strategy, because the decision making is according to whether or not the node's current state is proper to defend at this moment. In other words, the defending action may not always be the best choice for nodes in MANETs when we try to extend the average lifetime and reduce the compromising probability of MANETs.

When the attacker launches dynamic attack, the dynamic change of the target node's actions in the first 50 steps is presented in Fig. 10. In this situation, the attacking target is also chosen dynamically in each step by the attacker. Compared with Fig. 9, there is no node being attacked in some steps, because the attacker does not attack continuously. The simulation result illustrates that even if the node is attacked in one step, it does not choose the defending action all the time with the optimal policy. In addition, in both Fig. 9 and Fig. 10, the nodes' actions are not 


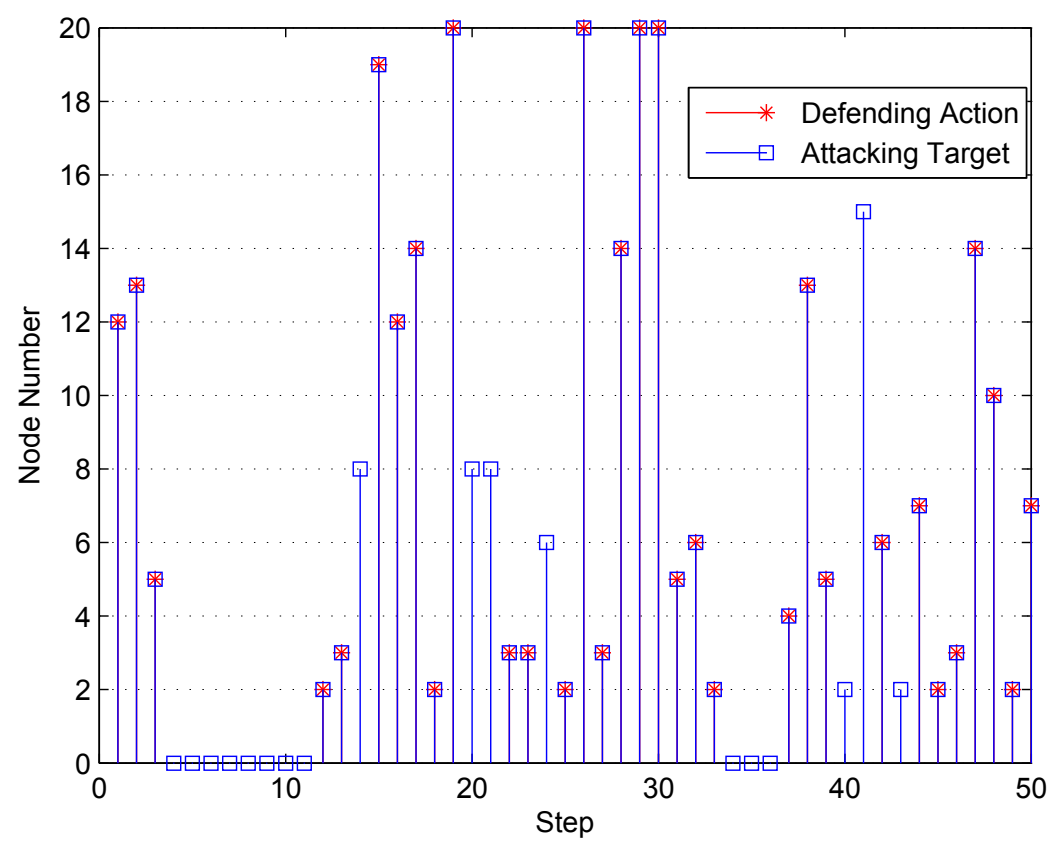

Figure 10: Attacking target and defence action under major player's dynamical attack.

defending when they are not the attacking targets according to the optimal policy we got. Each node tries to control the energy consumption efficiently. So the lifetime of MANET can be extended.

\subsubsection{Performance with Limited Energy}

Network lifetime is one of the key performance metrics in the MANET. Here, we consider the network lifetime with two constraints. In these constraints, the setup of the probabilities is designed for the evaluation of the performance with the proposed scheme on the network lifetime [69]. The first constraint is that if one node's energy consumption reaches $90 \%$, the node cannot work well. When there are more than $70 \%$ nodes in the MANET that cannot work well, the MANET will be considered dead. The second constraint is that if the node's loss of security value reaches $80 \%$, 


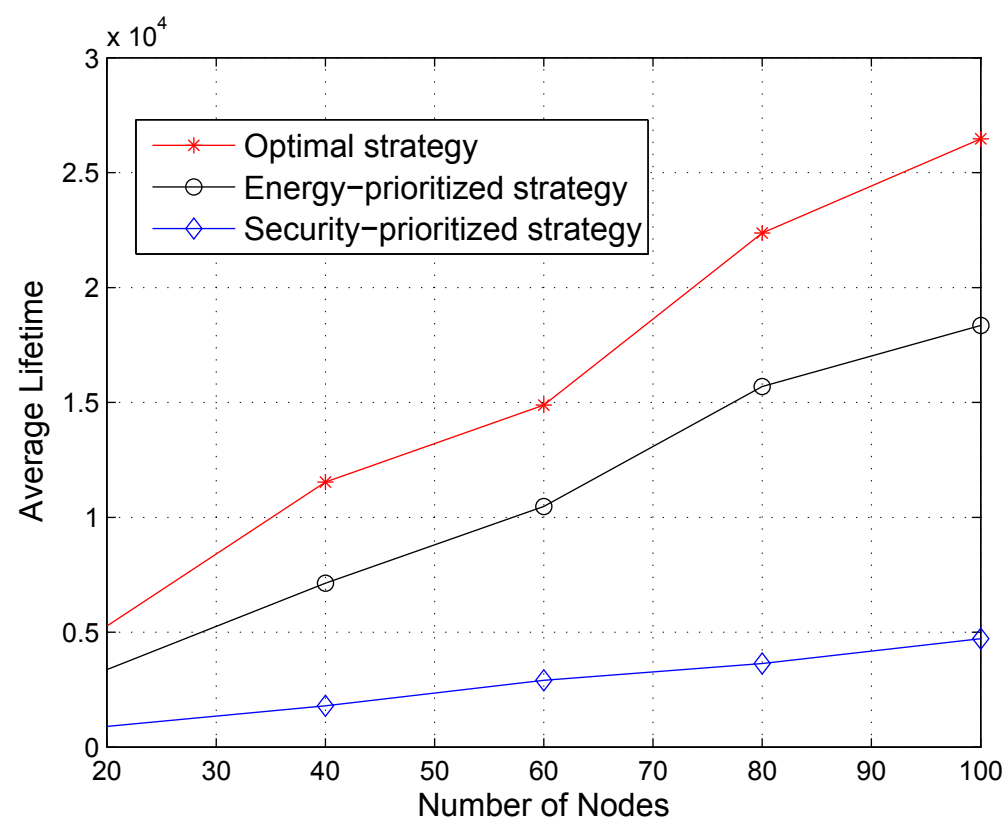

Figure 11: Comparison of average lifetime with different numbers of nodes (limited energy).

the node is compromised. The network is deemed compromised when there are more than $50 \%$ nodes in the MANET compromised.

We assume that each node has the same initial value of the combination of energy and security. Fig. 11 illustrates the average lifetime of a MANET when the number of nodes increases from 20 to 100. With the increase of the number of available nodes, the lifetime of MANET will increase as well. Compared to the two other strategies, the proposed scheme has the best average lifetime due to the optimal strategy. The proposed method is more suitable for the MANET with larger number of nodes when they encounter dynamical attacks from the major player.

In these simulations, we also investigate the compromising probability of the MANET. For evaluating the performance of the proposed scheme, we assume that when the number of compromised nodes is up to $15 \%$ of the total number of the nodes in the MANET, the whole MANET can be considered to be compromised. Fig. 12 


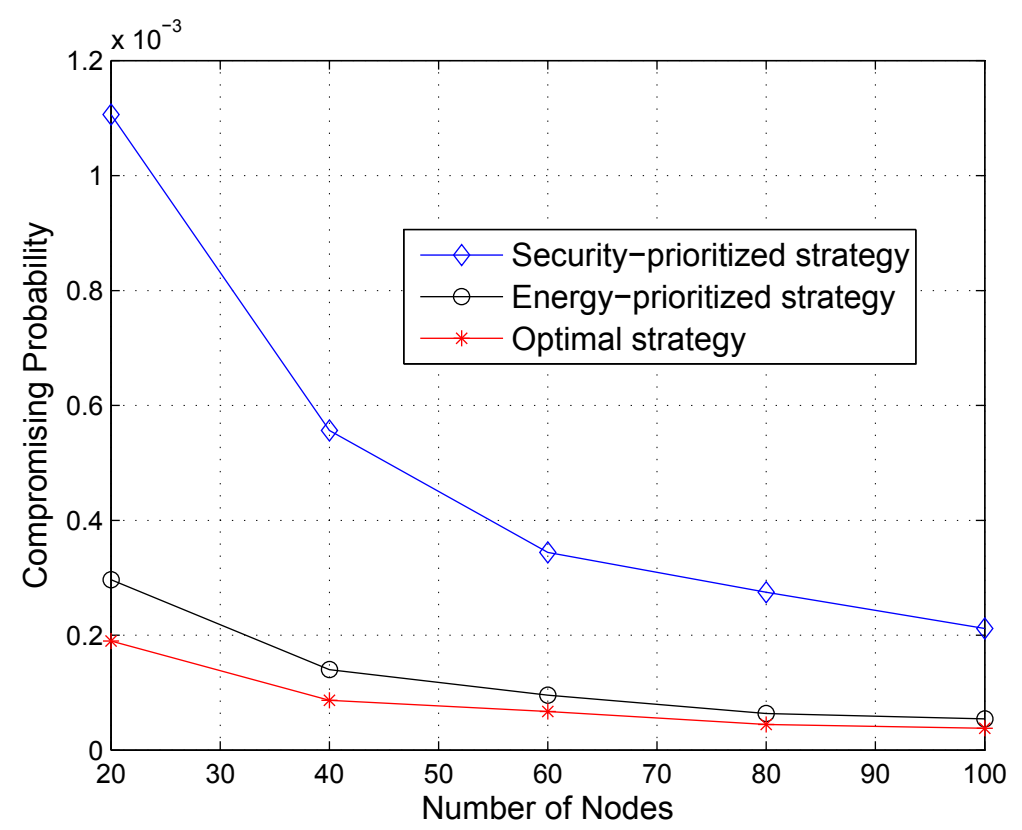

Figure 12: Comparison of compromising probabilities with different numbers of nodes (limited energy).

illustrates a downward trend in compromising probabilities when the total number of nodes increases in the MANET. When the minor players adopt the optimal strategy, the MANET's compromising probability is always lower than the compromising probabilities when they adopt the two other strategies.

\subsubsection{Performance with Sufficient Energy}

In some situations, the nodes in the MANET may be supplied with sufficient energy (e.g., vehicular ad-hoc networks). So we only consider the security value loss as the criterion to determine the lifetime. The results in Fig. 13 and Fig. 14 indicates that, although security-prioritized strategy can bring longer lifetime and lower compromising probability than the energy-prioritized strategy in this situation, our optimal strategy can provide the best performance for the MANET among these three strategies. 


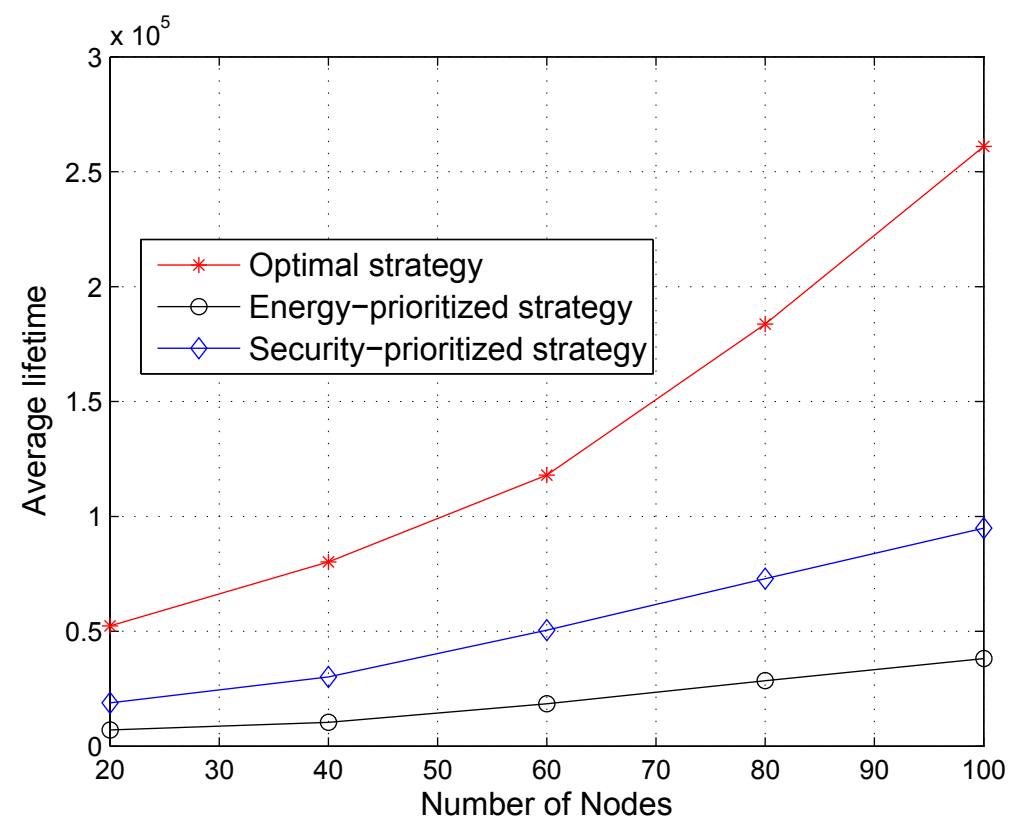

Figure 13: Comparison of average lifetimes with different numbers of nodes (sufficient energy).

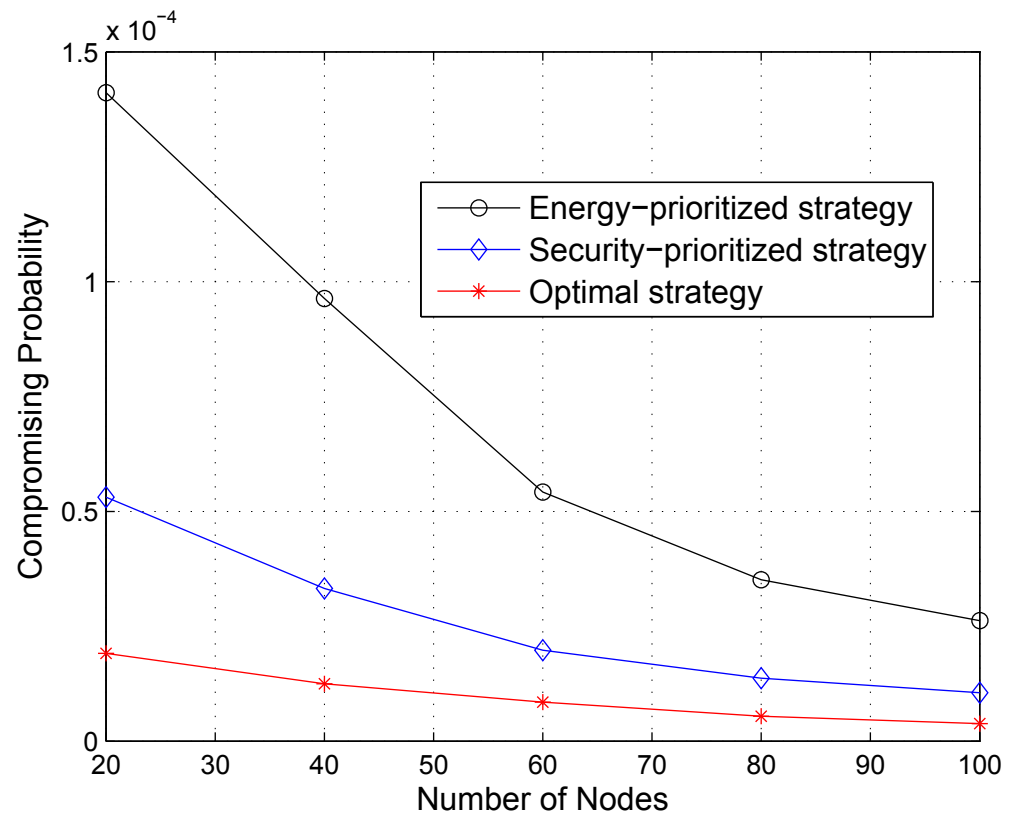

Figure 14: Comparison of compromising probabilities with different numbers of nodes (sufficient energy). 


\subsection{Summary}

In this chapter, we firstly introduced the simulation scenarios: one malicious node is trying to attack the MANET which consists of $\mathrm{N}$ nodes. Each of these nodes is equipped with IDS sensor to detect the intrusion. Secondly, we present how to obtain the stochastic distributed optimal defending strategy with an example. In this study, the malicious node is considered as a smart attacker which can choose its strategy to maximize its income and minimize its energy consumption. Each minor player in this MANET makes its decision independently to choose the defending strategy based on the detection of the IDS sensor and its anticipation to the whole MANET. We suppose that there is limited information sharing among the minor players for the defending. So using the approximation method to anticipate could improve the efficiency to make the decision.

Then we present the simulation results to validate our analytical results and effectiveness of the proposed game theoretic approach for security and energy consumption in mobile ad-hoc networks. The performances of a representative node which adopts the optimal strategy are compared along with the performance of adopting other two strategies. The average costs, the average lifetime and the compromising probability of the MANET are also compared and discussed. Additionally, in the simulations, we not only consider the situation of the nodes in the MANET with limited energy such as the sensor networks, but also consider the situation of the nodes with sufficient energy, such as vehicular ad-hoc networks. The simulation results illustrate that using the optimal strategy, the MANET's compromising probability is always much lower and the average lifetime could be extended. 


\section{Chapter 5}

\section{Mean Field Game Method for Security in Cognitive Radio Mobile Ad-hoc Networks}

\section{$5.1 \quad$ Introduction}

Cognitive radio [38] as an opportunistic communication technology is designed to improve the utilization of the available licensed bandwidth for unlicensed users.

As shown in Fig. 15, the usage of the limited spectrum is very inefficient $[1,70]$. Cognitive radio technology helps network users make smart decisions on the usage of spectrum and operate parameters based on the sensed spectrum dynamics and actions adopted by other users.

In CR-MANETs, mobile nodes can not only autonomously organize and communicate with each other over bandwidth-constrained wireless links, but also have the capacity to sense the spectrum and choose the proper channels for data transmission.

Due to the lack of any central authority and shared wireless medium, the unique characteristics of CR-MANETs present some new challenges to security design [2]. Many security threats exist in CR-MANETs, such as denial of service, black hole, resource consumption, information disclosure, and interference [3, 4]. 


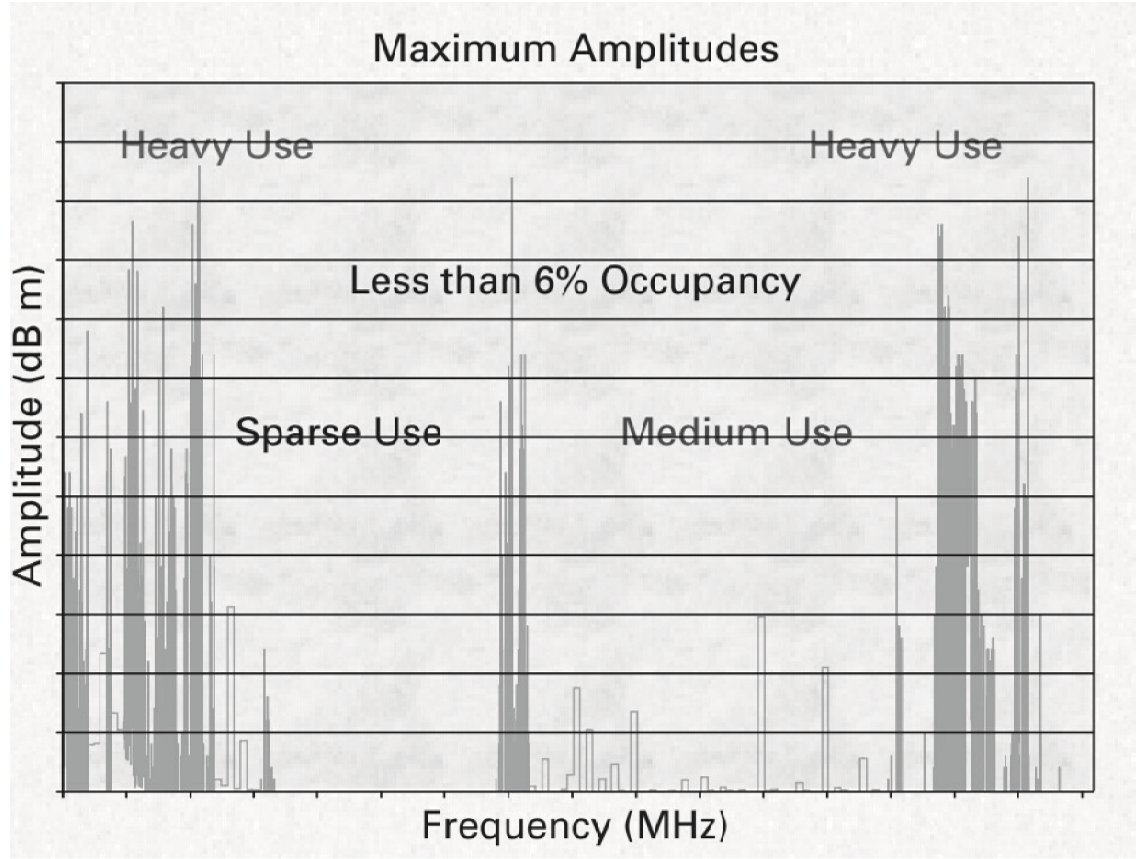

Figure 15: Spectrum usage (from FCC Report [1]).

The authors in [71] considered the spectrum sensing data falsification (SSDF) attacks to CR-MANETs. The intruders can send false local spectrum sensing results in cooperative spectrum sensing and SSDF may lead to incorrect spectrum sensing decisions by CRs. In [39], the researchers introduced two kinds of attacks from the exogenous attackers. One is the Jamming attack and the other is the Incumbent Emulation attack. In the jamming attack, an attacker always floods the sensed channel with white/colored noise. Energy detection for spectrum sensing can be used to specify this kind of jamming attack.

Recently, game theoretic approaches have been proposed to improve network security $[34,35]$. Game theory is a useful tool to provide a mathematical framework for modeling and analyzing decision problems, since it can address problems where multiple players with contradictory goals or incentives compete with each other. In game theory, one player's outcome depends not only on his/her decisions, but also 
on the decisions of other players. Similarly, the success of a security scheme in CRMANETs depends not only on the actual defense strategies, but also on the actions taken by the attackers.

As shown in Fig. 16, the authors introduced a comprehensive treatment of game theory oriented toward applications to cognitive radio networks.

In [40], the authors extended the mean field game framework to a hierarchical interacting system for the cognitive wireless networks, which consisted of a finite number of primary users and a large number of secondary users. B. Wang et al. introduced an anti-jamming stochastic game for cognitive radio networks. The anti-jamming defense was proposed using the minimax-Q learning algorithm. The performance of using the optimal stationary policy was improved as described in [41].

Although some excellent research has been done on addressing the security issues in CR-MANETs using game theoretic approaches, most of the existing work only considered a security game model with an attacker and a defender. In this chapter, we consider the security problems in CR-MANETs using the mean field game theory [59] which can provide a powerful mathematical tool for problems with a large number of players [60].

To the best of our knowledge, using mean field game theoretic approach for security in CR-MANETs has not been considered in the existing works. In this work, a dynamic mean field game theoretic approach is proposed to enable an individual node in CR-MANETs to make strategic security defence decisions without centralized administration. 


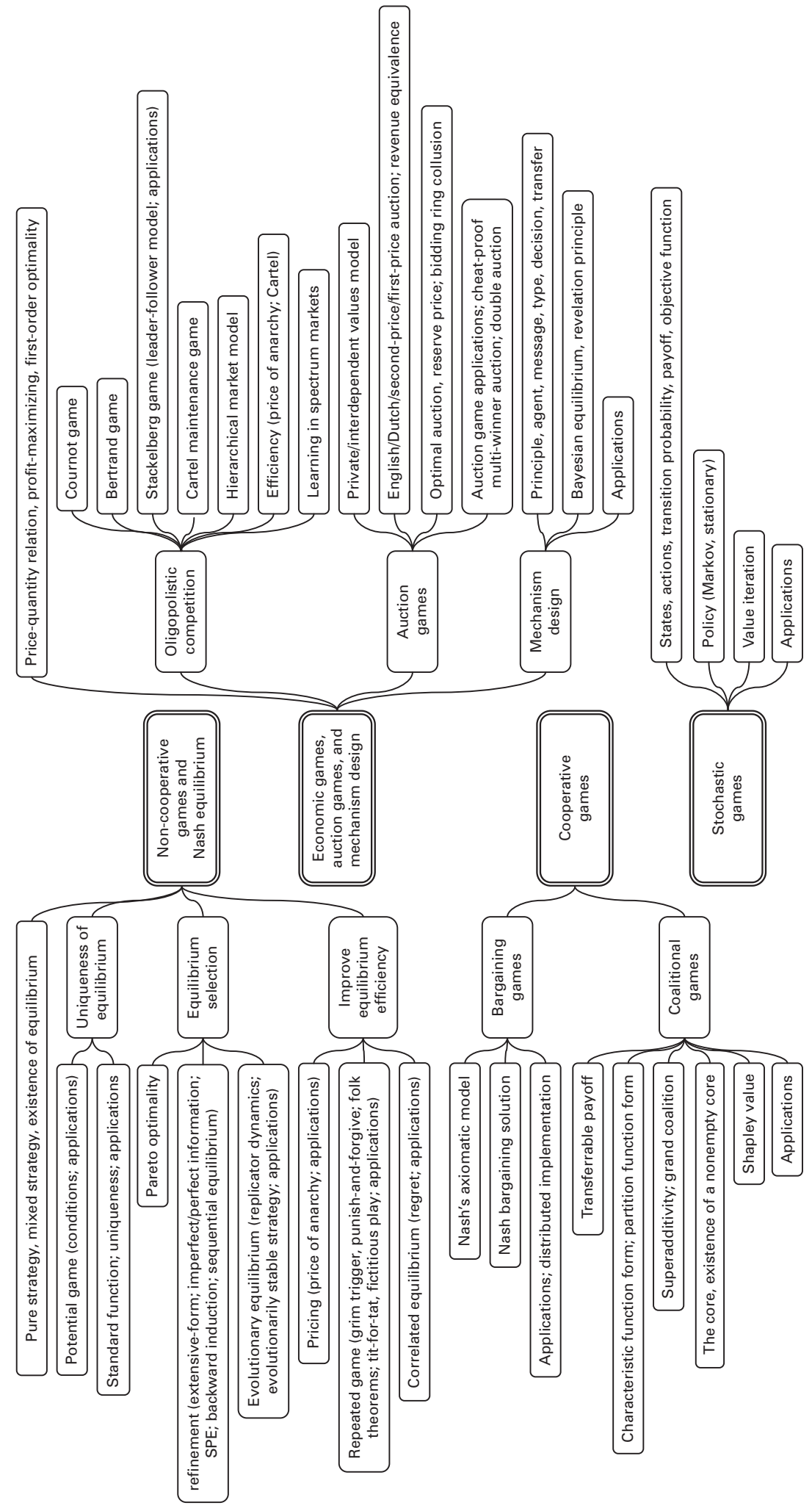

Figure 16: Four categories of the game-theoretic spectrum sharing approaches [1]. 


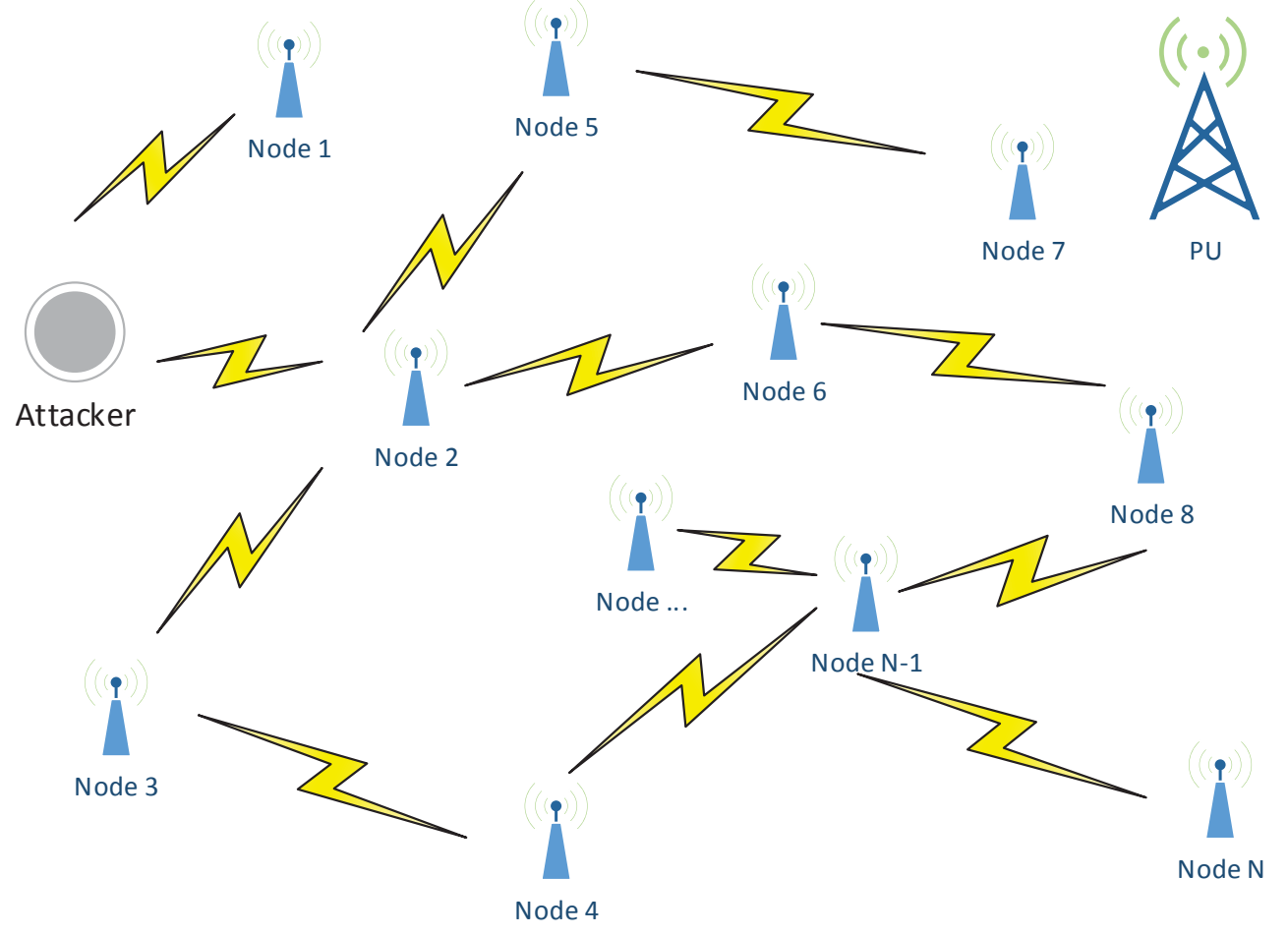

Figure 17: System consists of a CR-MANET with $N$ nodes and an attacker.

\subsection{Model Description and Formulation in CR- MANETs}

In this section, a system which contains an $N$-node CR-MANET and an attacker is presented. Based on the discussion, the security problem in this system is formulated as an $N+1$ mean field game. In addition, we suppose that there is a PU which works dynamically using the licensed spectrum.

\subsubsection{Description of System Model}

We suppose that a CR-MANET consists of $N$ mobile nodes. All these nodes are SUs, so they are secondary user networks. When the PU is not using the spectrum, the SUs can use the spectrum. However, at that moment, this CR-MANET can be 
disturbed by an attacker which loads the jamming attack dynamically. The system is illustrated in Fig. 17.

All these legitimate nodes in this CR-MANET are independent and mobile, since there is no centralized administration in the CR-MANET. Additionally, all these legitimate nodes could use the spectrums temporarily when the licensed spectrum is unused by the PU to improve the usage efficiency of the limited spectrum.

The PU's "interference" and the attack from the attackers could influence the spectrum usage of the SUs. There are several techniques, such as energy detection, feature detection, matched filtering, and coherent detection [1], which could be used to help these independent SUs distinguish these influences.

There are three cases of detecting results for the SUs:

- Case 1: PU is using the spectrum and there is no jamming. SUs wait until the PU leaves;

- Case 2: PU is not using the spectrum and there is no jamming at the same time. SUs can use the spectrum at once;

- Case 3: PU is not using the spectrum but there is jamming attack for the SUs to use the spectrum. The SUs tries to use optimal strategy to defend.

We model this system as an $N+1$ mean field game model as follows. These legitimate nodes in CR-MANETs are considered as the $N$ minor players in the mean field game. Meanwhile, the attacker, which tries to attack the whole CR-MANET, is considered as a major player.

\subsubsection{Energy Detection Spectrum Sensing Method}

As we mentioned above, there are many methods that can be used to detect the jamming attack. For implementation simplicity, we will consider the energy detection 


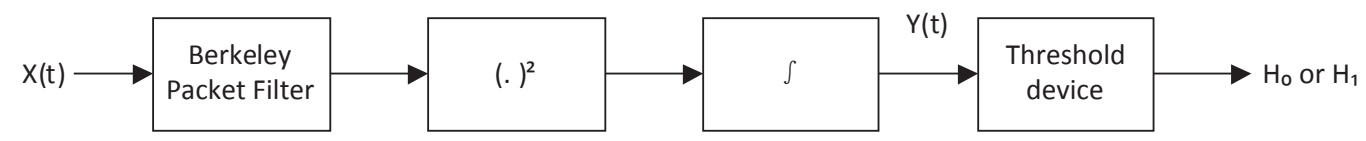

Figure 18: Block diagram of an energy detector.

spectrum sensing method.

As shown in Fig. 18, the energy detector consists of a input bandpass filter, a squaring device, an integrator, and a comparator. A center frequency $f_{s}$ and the bandwidth of interest $W$ are chosen as the parameters of the input bandpass filter. The squaring device following the filter measures the received energy. The integrator works with the observation interval $T$. The output of the integrator is compared with a threshold $\lambda$ to decide whether the jamming attack is present in the spectrum.

The goal of spectrum sensing is to decide between the following two hypotheses:

$$
X(t)=\left\{\begin{array}{cc}
n(t), & H_{0} \\
h \cdot S_{J}(t)+n(t), & H_{1}
\end{array}\right.
$$

where $X(t)$ is the signal received by the SU, $S_{J}(t)$ is the intruder's jamming attack signal, $n(t)$ is the additive white Gaussian noise (AWGN) and $h$ is the amplitude gain of the channel.

We let $\gamma$ denote the signal-to-noise ratio (SNR).

$Y$ denotes the output of the integrator and has the following distribution

$$
Y=\left\{\begin{array}{cc}
X_{2 T W}^{2}, & H_{0} \\
X_{2 T W}^{2}(2 \gamma), & H_{1}
\end{array}\right.
$$


where $X_{2 T W}^{2}$ and $X_{2 T W}^{2}(2 \gamma)$ denote random variables with central and non-central chi-square distributions, respectively, each with $2 T W$ degrees of freedom and a noncentrality parameter of $2 \gamma$ for the latter distribution. For simplicity, we assume that time-bandwidth product, $T W$, is an integer number, which is denoted by $m$. Under Rayleigh fading, $\gamma$ would have an exponential distribution, so in this case the average SNR $\bar{\gamma}$ is used instead.

In addition, $h$ is not deterministic. Therefore, according to [71]

$$
Y=\left\{\begin{array}{cc}
X_{2 T W}^{2}, & H_{0} \\
X_{(2 T W-2)}^{2}+e_{(2 \bar{\gamma}+2)}, & H_{1}
\end{array}\right.
$$

where $e_{(2 \bar{\gamma}+2)}$ is a random variable having the exponential distribution with parameter $(2 \bar{\gamma}+2)$ and $X_{(2 T W-2)}^{2}$ is a random variable with non-central chi-square distribution with $(2 T W-2)$ degrees of freedom. In our proposed mean field scheme, the $Y$ using for the comparison with threshold combines the mean field value from the other SUs in the CR-MANET. We define that

$$
Y_{\text {mean }}=Y+\mu I^{(N)}(t)
$$

where $\mu$ is the weight of $I^{(N)}(t)$.

\subsubsection{Major Player and Minor Players in CR-MANETs}

For the attacker, it is intelligent and equipped with cognitive radio. So it can load the jamming attack to the spectrum which is being used by the legitimate nodes. The attacker needs to make decisions to choose the proper target and load the attack, since it has to consume the limited system resources (such as power). Its purpose is to maximize the destruction to the communication of the whole CR-MANET as 
much as possible.

The major player's state space is denoted as $S_{0}=\left\{1, \cdots, L_{0}\right\}$. Its action space is defined as $A_{0}=\left\{1, \cdots, M_{0}\right\}$. Meanwhile, the minor players' state space and action space are $S=\{1, \cdots, L\}$ and $A=\{1, \cdots, M\}$, respectively. The major player $\mathcal{A}_{0}$ 's state is $s_{0}(t)$ and its action is $a_{0}(t) . s_{i}(t)$ and $a_{i}(t)$ denote the state and the action of a representative legitimate minor player $\mathcal{A}_{i}, i \in(1, \cdots, N)$, respectively.

\subsubsection{States and Transition Laws in CR-MANETs}

The major player's state is defined as a combination of power and information assets, which can be denoted by $\alpha_{\mathrm{E}_{0}} E_{0}+\alpha_{I} I_{0}$ [65]. Here, $\alpha_{\mathrm{E}_{0}}$ and $\alpha_{I}$ represent the weights of power and the spectrum information assets, respectively. Meanwhile, the minor players' state is defined as a combination of power and security spectrum assets, which is denoted by $\alpha_{\mathrm{E}_{i}} E_{i}+\alpha_{S} S_{i}$.

A limiting process $\theta(t)$ is used to approximate the random measure process $I^{(N)}(t)$ to overcome the fundamental complexity. The updating rule of the limiting process $\theta(t)$ is proposed as $(61)$, in which $\theta(0)=\theta_{0}$.

Additionally, $Q_{0}\left(z \mid y, a_{0}\right)$ and $Q\left(z \mid y, a_{i}\right)$ represent the state transition laws of the major player and representative minor player, respectively. The state transition of the major player is specified by

$$
Q_{0}\left(z \mid y, a_{0}\right)=P\left(s_{0}(t+1)=z \mid s_{0}(t)=y, a_{0}(t)=x_{0}\right),
$$

where $y, z \in S_{0}$ and $x_{0} \in A_{0}$. For the minor player $\mathcal{A}_{i}$, the state transition law is determined by

$$
Q\left(z \mid y, a_{i}\right)=P\left(s_{i}(t+1)=z \mid s_{i}(t)=y, a_{i}(t)=x\right)
$$


where $y, z \in S$, and $x \in A$.

\subsubsection{Cost Functions of CR-MANETs}

We define the instantaneous cost of the major player as follows:

$$
\begin{aligned}
& c_{0}\left(s_{0}(t), a_{0}(t), I^{(N)}(t)\right) \\
& =f_{0}\left(s_{0}(t), a_{0}(t)\right)-f\left(I^{(N)}(t)\right),
\end{aligned}
$$

where $f_{0}\left(s_{0}(t), a_{0}(t)\right)$ denotes the coupled power consumption when the major player

adopts different actions under various states. $f\left(I^{(N)}(t)\right)$ denotes the cost of the major player, which comes from the attacking. $f\left(I^{(N)}(t)\right)$ should also represent the average reflection of the whole mean field to the major player's attack.

We define the cost of a presentative minor player as follows:

$$
\begin{aligned}
& c_{i}\left(s_{i}(t), a_{i}(t), s_{0}(t), a_{0}(t), I^{(N)}(t)\right) \\
= & g_{i}\left(s_{i}(t), a_{i}(t)\right)-g_{0}^{i}\left(I^{(N)}(t), s_{0}(t), a_{0}(t)\right) .
\end{aligned}
$$

In the equation above, $g_{i}\left(s_{i}(t), a_{i}(t)\right)$ denotes the coupled cost when the presentative minor player adopts different actions under one state. $g_{0}^{i}\left(I^{(N)}(t), s_{0}(t), a_{0}(t)\right)$ represents the combined cost from the influence of the major player's state, action, and the reflection of the whole mean filed.

\subsubsection{Mean Field Equation System}

By using the mean field approximation approach,the mean field equation system can be defined as follows:

$$
\theta(t+1)=\varphi\left(s_{0}(t), \theta(t)\right),
$$




$$
\begin{aligned}
& v\left(s_{0}, \theta\right)=\min _{a_{0} \in A_{0}}\left\{c_{0}\left(s_{0}, a_{0}, \theta\right)+\Delta\right\}, \\
& w\left(s_{i}, s_{0}, \theta\right)=\min _{a_{i} \in A}\left\{c_{i}\left(s_{i}, a_{i}, s_{0}, \theta\right)+\Omega\right\},
\end{aligned}
$$

where $\Delta \quad=\quad \rho \sum_{k \in S_{0}} Q_{0}\left(k \mid s_{0}, a_{0}\right) v\left(k, \varphi\left(s_{0}, \theta\right)\right) \quad$ and $\quad \Omega \quad=$ $\rho \sum_{j \in S, k \in S_{0}} Q\left(j \mid s_{i}, a_{i}\right) Q_{0}\left(k \mid s_{0}, \hat{\pi}_{0}\right) w\left(j, k, \varphi\left(s_{0}, \theta\right)\right) . \quad \theta(t)$ represents a limiting process, which is used to approximate the random measure process $I^{(N)}(t)$ for a low complexity solution. (62) and (63) are the dynamic programming equations for the major player and a representative minor player, respectively. $c_{i}\left(s_{i}, a_{i}, s_{0}, \theta\right)$ is transformed from $c_{i}\left(s_{i}, a_{i}, s_{0}, a_{0}, \theta\right)$ in $(63)$.

\subsection{Approximation Method of the Mean Field Process}

We introduce the $L \times L$ matrix

$$
\begin{aligned}
& Q^{*}\left(s_{0}, \theta\right) \\
& =\left[\begin{array}{ccc}
Q\left(1 \mid 1, \hat{\pi}\left(1, s_{0}, \theta\right)\right) & \ldots & Q\left(L \mid 1, \hat{\pi}\left(2, s_{0}, \theta\right)\right) \\
Q\left(1 \mid 2, \hat{\pi}\left(2, s_{0}, \theta\right)\right) & \ldots & Q\left(L \mid 2, \hat{\pi}\left(2, s_{0}, \theta\right)\right) \\
\vdots & \ddots & \vdots \\
Q\left(1 \mid L, \hat{\pi}\left(L, s_{0}, \theta\right)\right) & \ldots & Q\left(L \mid L, \hat{\pi}\left(L, s_{0}, \theta\right)\right)
\end{array}\right] .
\end{aligned}
$$

The function $\varphi$ can be revised as

$$
\varphi\left(s_{0}, \theta\right)=\theta Q^{*}\left(s_{0}, \theta\right) .
$$


For ease of presentation, we assume the minor player has two states. The limiting process $\theta(t)$ should contain two vectors: $\theta(t)=\left\{\theta_{0}(t), \theta_{1}(t)\right\}$, where $\left(\theta_{0}(t)+\theta_{1}(t)=1\right)$. Here, $\theta_{0}(t)$ indicates the probability of the minor players' state $s_{i}=0$ and $\theta_{1}(t)$ indicates the probability of the minor players' state $s_{i}=1$. The updating rule for $\theta_{0}$ is

$$
\varphi=s_{0}\left(\theta_{0}\right)^{1 / 2}+\left(1-s_{0}\right)\left(\theta_{0}\right)^{2}
$$

where $\theta_{0} \in[0,1]$.

\subsubsection{Cost Functions with the Approximation of Mean Field Process}

We let $\gamma_{i} \alpha_{i}-\left(1-\gamma_{i}\right) \beta_{i}$ represent the value of security in a spectrum, which could be used by the representative minor player and let $\left(1-\gamma_{i}\right) \beta_{i}$ denote the reward the attacker gets from the representative minor player for its attacking action.

Considering the average effect of the mean field to the major player and the representative minor player, we get the new cost functions as follows:

$$
\begin{aligned}
& c_{0}\left(s_{0}(t), a_{0}(t), \theta(t)\right) \\
= & f_{0}\left(s_{0}(t), a_{0}(t)\right)-\theta(t) \sum_{i=1}^{N}\left(1-\gamma_{i}\right) \beta_{i}, \\
& c_{i}\left(s_{i}(t), a_{i}(t), s_{0}(t), a_{0}(t), \theta(t)\right) \\
= & g_{i}\left(s_{i}(t), a_{i}(t)\right)-\theta(t)\left[\gamma_{i} \alpha_{i}-\left(1-\gamma_{i}\right) \beta_{i}\right] .
\end{aligned}
$$

Finally, the function $\varphi$ can be revised as (65). 


\subsubsection{Mixed Strategies and State Transition Laws}

Table 4: Setups of the Elements for Both the Major Player and the Representative Minor Player in CR-MANETs

\begin{tabular}{|c|c|c|c|c|c|c|c|}
\hline & \multicolumn{7}{|c|}{ Setups } \\
\hline $\begin{array}{l}\text { major player's state s- } \\
\text { pace }\end{array}$ & \multicolumn{7}{|c|}{$S_{0}=\{0,1\}$} \\
\hline $\begin{array}{l}\text { major player's action s- } \\
\text { pace }\end{array}$ & \multicolumn{7}{|c|}{$A_{0}=\{0,1\}$} \\
\hline $\begin{array}{l}\text { major player's state } \\
\text { transition matrices }\end{array}$ & \multicolumn{2}{|c|}{$Q_{0}\left(z \mid y, a_{0}=0\right)=$} & $\begin{array}{cc}0.7 & 0.3 \\
0.05 & 0.95\end{array}$ & \multicolumn{2}{|c|}{,$Q_{0}\left(z \mid y, a_{0}=1\right)=$} & $\begin{array}{r}0.9 \\
0.01\end{array}$ & $\begin{array}{c}0.1 \\
0.99\end{array}$ \\
\hline $\begin{array}{l}\text { major player's cost } \\
\text { function }\end{array}$ & \multicolumn{7}{|c|}{$c_{0}\left(s_{0}, a_{0}, \theta\right)=f_{0}\left(s_{0}, a_{0}\right)-\theta_{0} \sum_{i=1}^{N}(1-\gamma) \beta_{i}$} \\
\hline $\begin{array}{l}\text { major player's cost ma- } \\
\text { trix }\end{array}$ & \multicolumn{7}{|c|}{$R_{0}=\left[\begin{array}{ll}c_{0}\left(s_{0}=0, a_{0}=0, \theta\right) & c_{0}\left(s_{0}=0, a_{0}=1, \theta\right) \\
c_{0}\left(s_{0}=1, a_{0}=0, \theta\right) & c_{0}\left(s_{0}=1, a_{0}=1, \theta\right)\end{array}\right.$} \\
\hline $\begin{array}{l}\text { a representative minor } \\
\text { player's state space }\end{array}$ & \multicolumn{7}{|c|}{$S_{i}=\{0,1\}$} \\
\hline $\begin{array}{l}\text { a representative minor } \\
\text { player's action space }\end{array}$ & \multicolumn{7}{|c|}{$A_{i}=\{0,1\}$} \\
\hline $\begin{array}{l}\text { a representative minor } \\
\text { player's state transition } \\
\text { matrix }\end{array}$ & \multicolumn{2}{|c|}{$Q\left(z \mid y, a_{i}=0\right)=$} & $\begin{array}{cc}0.8 & 0.2 \\
0.03 & 0.97\end{array}$ & \multicolumn{2}{|c|}{,$Q\left(z \mid y, a_{i}=1\right)=$} & $\begin{array}{c}0.9 \\
0.01\end{array}$ & $\begin{array}{c}0.1 \\
0.99\end{array}$ \\
\hline $\begin{array}{l}\text { a representative minor } \\
\text { player's cost function }\end{array}$ & \multicolumn{7}{|c|}{$c_{i}\left(s_{i}, a_{i}, a_{0}, \theta\right)=g_{i}\left(s_{i}, a_{i}\right)-\theta\left[\gamma_{i} \alpha_{i}-\left(1-\gamma_{i}\right) \beta_{i}\right]$} \\
\hline $\begin{array}{l}\text { a representative minor } \\
\text { player's cost matrices }\end{array}$ & $R_{1}=$ & $\begin{array}{l}c_{i}(0,0,0, \theta) \\
c_{i}(1,0,0, \theta)\end{array}$ & $\begin{array}{l}c_{i}(0,1,0, \theta) \\
c_{i}(1,1,0, \theta)\end{array}$ &,$R_{2}=$ & $\begin{array}{l}c_{i}(0,0,1, \\
c_{i}(1,0,1,\end{array}$ & $c_{i}((1$ & $\begin{array}{l}, 1,1, \theta) \\
, 1,1, \theta)\end{array}$ \\
\hline
\end{tabular}

Based on the above mean field game modeling and analysis, we use an example to show the stochastic distributed optimal defending strategy in CR-MANETs.

For ease of presentation, suppose the setups as showing in Table $4 . a_{0}=0$ means the major player takes the action "Attack", while $a_{0}=1$ denotes the action "Not Attack". 
These major player's state transition matrices stand for the probabilities of the major player changing from one state to another. For example, when the major player's state is $s_{0}=0$, if it chooses action $a_{0}=0$, it can keep the state $s_{0}=0$ with probability 0.7 in the next step, and it can change to the state $s_{0}=1$ with probability 0.3 . The setup of the above nodes' transition matrices and cost matrices is a non-trivial task for the proposed scheme.

In the definition of the major player's cost function, $f_{0}\left(s_{0}, a_{0}\right)=\left(2-s_{0}\right)(1-$ $\left.a_{0}\right), N=40, \gamma=0.8$, and $\beta_{i}=0.25$. When $\theta_{0}$ in major player's cost function tends to be 1, it means most of the minor players are in positive defending states. If the major player chooses to attack under this situation, the successful defending rate $\gamma$ should be larger and the reward $\sum_{i=1}^{N}(1-\gamma) \beta_{i}$ should be smaller. So $c_{0}\left(s_{0}, a_{0}, \theta_{0}\right)$ should get a greater value.

During the value iteration, we choose $\theta_{0}$ starting from 0.7 for the iteration. We can get the matrix:

$$
Q_{0}=\left[\begin{array}{ll}
Q_{0}\left(0 \mid 0, \pi_{0}\right) & Q_{0}\left(1 \mid 0, \pi_{0}\right) \\
Q_{0}\left(0 \mid 1, \pi_{0}\right) & Q_{0}\left(1 \mid 1, \pi_{0}\right)
\end{array}\right]=\left[\begin{array}{cc}
0.7 & 0.3 \\
0.01 & 0.99
\end{array}\right] .
$$

For the representative minor player, the state space $S_{i}$ and action space $A_{i}$ are defined in Table 4. Since the state of the representative minor player is a combination of energy asset and security asset, $s_{i}=0$ means the node is safe. It has full energy to do the positive defense and to keep the security value for itself. Meanwhile, $s_{i}=1$ denotes the node is unsafe, and it can only do passive defense during the game process. $a_{i}=0$ means the "Defending" action. The node can move and change transmission parameters and $a_{i}=1$ denotes the "Not Defending" action. We set the representative minor player's state transition matrix and the representative minor 
player's cost function in Table 4 , where $g_{i}\left(s_{i}, a_{i}\right)=\left(1.8-s_{i}\right)\left(1-a_{i}\right), N=40, \gamma_{i}=$ $0.8, \alpha_{i}=1$, and $\beta_{i}=1.5$.

Table 5: Utility Matrix of the Representative Minor Player in CR-MANETs

\begin{tabular}{|c|c|c|}
\hline & Defending & Not Defending \\
\hline Attack & $g_{i}\left(s_{i}, 0\right)-\theta_{d}\left[\gamma_{i} \alpha_{i}-\left(1-\gamma_{i}\right) \beta_{i}\right]$ & $g_{i}\left(s_{i}, 1\right)+\theta_{d} \beta_{i}$ \\
\hline Not attack & $g_{i}\left(s_{i}, 0\right)$ & 0 \\
\hline
\end{tabular}

Table 5 illustrates the cost matrix of the representative minor player $\mathcal{A}_{i}$ in the strategic form.

Based on the cost matrices of the representative minor player in Table 4, we can get $R_{1}=\left[\begin{array}{cc}1.8-0.3 \theta & 2.5 \theta \\ 0.8-0.3 \theta & 2.5 \theta\end{array}\right]$, and $R_{2}=\left[\begin{array}{cc}1.8 & 0 \\ 0.8 & 0\end{array}\right]$.

To obtain the optimal policy of the representative minor player, we also choose $\theta_{0}$ starting from $\theta_{0}=0.7$ and updated with the same evolution rule.

Then we can get the state transition law of the representative minor player as follows:

$$
Q^{1}=\left[\begin{array}{ll}
Q(0 \mid 0, \pi) & Q(1 \mid 0, \pi) \\
Q(0 \mid 1, \pi) & Q(1 \mid 1, \pi)
\end{array}\right]=\left[\begin{array}{cc}
0.9 & 0.1 \\
0.03 & 0.97
\end{array}\right]
$$

when $s_{0}=0$, and

$$
Q^{2}=\left[\begin{array}{ll}
Q(0 \mid 0, \pi) & Q(1 \mid 0, \pi) \\
Q(0 \mid 1, \pi) & Q(1 \mid 1, \pi)
\end{array}\right]=\left[\begin{array}{cc}
0.9 & 0.1 \\
0.01 & 0.99
\end{array}\right]
$$

when $s_{0}=1$. The result illustrates that the optimal transition matrices are not the same for the representative minor player when the major player has different states. 
In a CR-MANET, it may be hard to judge what state the attacker is, but it may be easy to sense the attacker's attack using the cognitive radio technique.

Using the method in [60], we define the function $\varphi\left(s_{0}, \theta\right)=\theta Q^{*}\left(s_{0}, \theta\right)$, where $Q^{*}\left(s_{0}, \theta\right)$ is defined in $(64)$. We get

$$
Q^{*}\left(s_{0}, \theta\right)=\left[\begin{array}{cc}
Q\left(0 \mid 0, \hat{\pi}\left(0, s_{0}, \theta\right)\right) & Q\left(1 \mid 0, \hat{\pi}\left(0, s_{0}, \theta\right)\right) \\
Q\left(0 \mid 1, \hat{\pi}\left(1, s_{0}, \theta\right)\right) & Q\left(1 \mid 1, \hat{\pi}\left(1, s_{0}, \theta\right)\right)
\end{array}\right] .
$$

So the updated matrices of $\theta$ are

$$
Q^{*}\left(s_{0}=0, \theta\right)=\left[\begin{array}{cc}
0.9 & 0.1 \\
0.02 & 0.98
\end{array}\right]
$$

and

$$
Q^{*}\left(s_{0}=1, \theta\right)=\left[\begin{array}{cc}
0.9 & 0.1 \\
0.01 & 0.99
\end{array}\right]
$$

\subsection{Simulation Results and Discussions}

We consider the following simulation scenarios: A CR-MANET consists of $N$ secondary-user nodes, each of which is equipped with cognitive radio.

There is an intelligent attacker node to attack this CR-MANET dynamically when the PU is not using the spectrum. We suppose that the average usage of a spectrum by the PU is $30 \%[72]$.

At the start of the simulation, the attacker in this CR-MANET starts with a dynamic state $s_{0} \in S_{0}$. It evolutes with its optimal updating rule and attacks the 


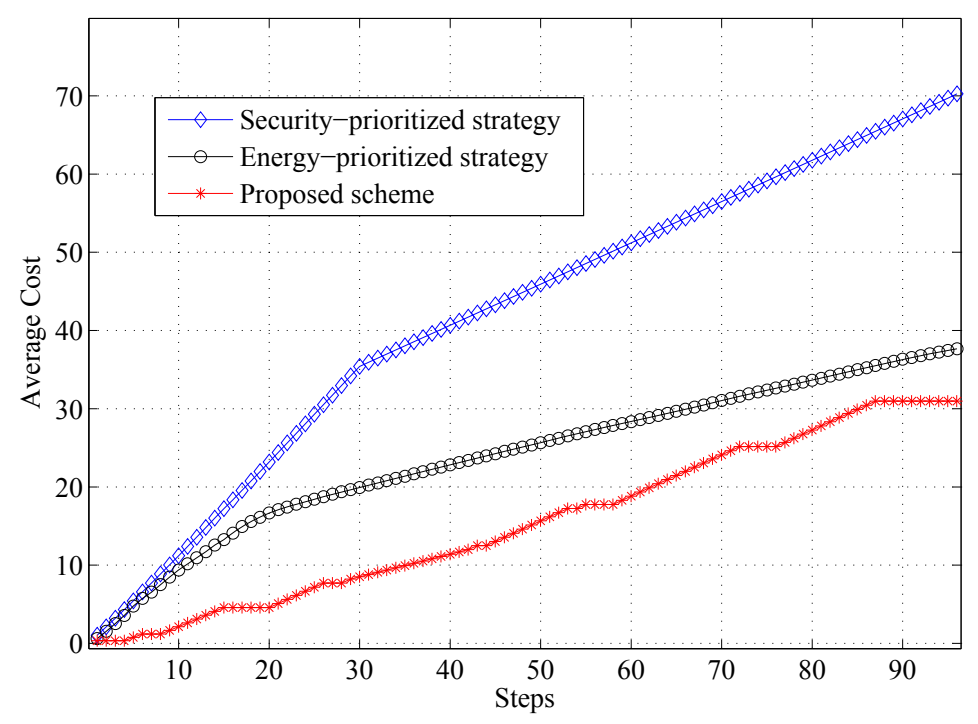

Figure 19: Average cost comparison among the representative minor player with security-prioritized strategy, energy-prioritized strategy and optimal strategy.

legitimate node randomly. The legitimate nodes' states start with safe and full power and then they are updated with one of the two optimal updating rules, which are based on the influence of the attacker. The legitimate nodes can detect the actions of the attacker using the cognitive radio technology, which is equipped in each node.

\subsubsection{Average Costs of Secondary Users}

In Fig. 19, the accumulated average costs with different strategies are compared. Here we consider three strategies, the security-prioritized strategy, the energy-prioritized strategy, and the optimal strategy.

In the energy-prioritized strategy, energy is the primary concern of the mobile nodes (e.g., in commercial scenario) [67], while security is the primary concern in the security-prioritized strategy (e.g., in military scenario) [68]. We perform the simulation 200 times and calculate a representative legitimate node's average cost of each step. 


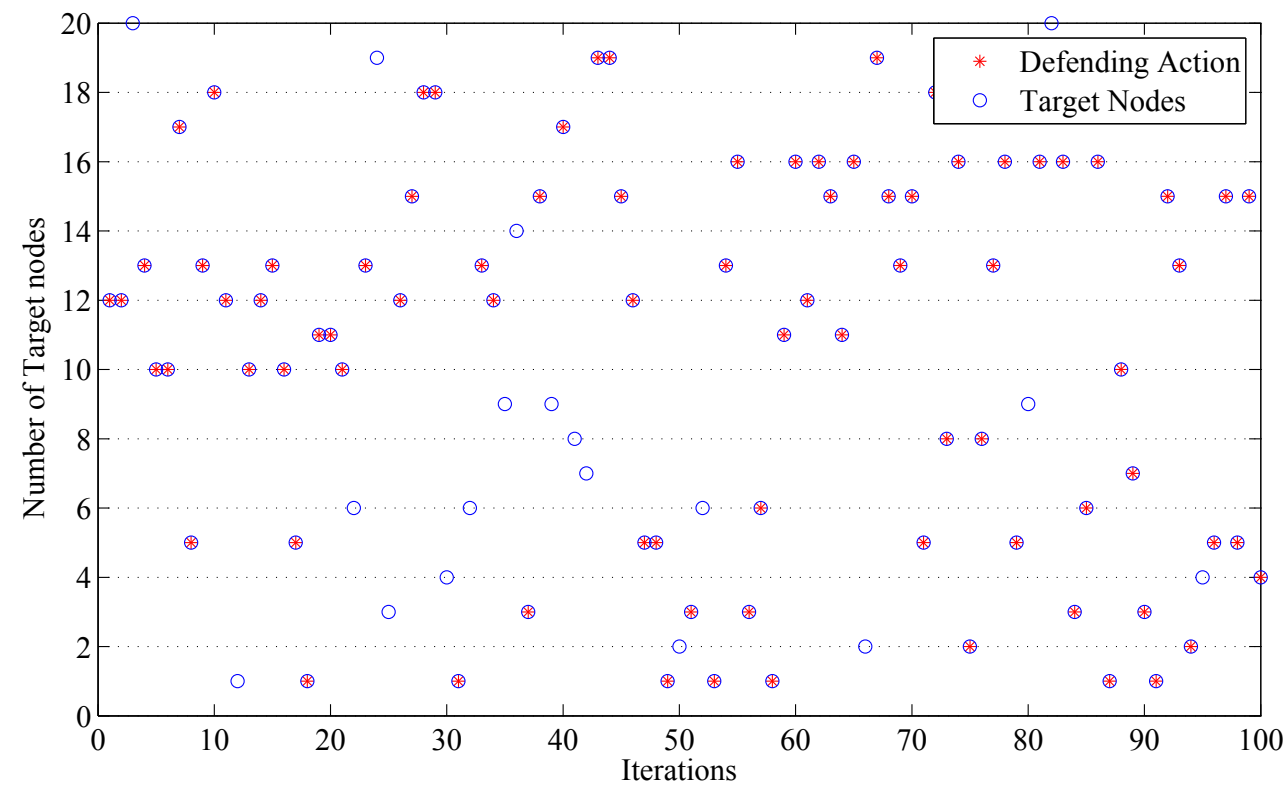

Figure 20: Attacking target and defence action under major player's dynamical attack.

As shown in Fig. 19, we can see that the proposed scheme has the lowest cost compared with the other two strategies. In other words, each CR-MANET node can greatly reduce energy consumption and loss of security in the spectrum with the optimal strategy.

\subsubsection{Defence Actions According to Optimal Strategy}

In the simulation, we study the representative CR-MANET node's defence actions when the attacker launches dynamic attack to the system. We choose a CR-MANET with 20 nodes $(N=20)$ and capture all the nodes' actions in each step. All the CR-MANET nodes use the optimal strategy derived in our scheme respectively.

In Fig. 20, the attacker loads the dynamic jamming attack and it chooses a node as the attacked target dynamically. The dynamic changes of the targeted node's action with the proposed scheme in the first 100 steps are recorded for observation. 
As shown in Fig. 20, the node being attacked in each step does not always choose the defending action with the optimal strategy, since the decision making is according to whether or not the node's current state is proper to defend at that moment. In other words, the defending action may not always be the best choice for nodes in CRMANETs when we try to extend the average lifetime of CR-MANETs. There is no node being attacked in some steps, because the attacker does not attack continuously. The simulation result illustrates that even if the node is being attacked in one step, it does not choose the defending action all the time with the optimal policy. In addition, the nodes' actions are not defending when they are not the attacked targets according to the optimal policy we got. Each node tries to control the energy consumption efficiently. So the lifetime of the CR-MANET can be extended.

\subsubsection{False Alarm Probability and Missing Detection Prob- ability}

In our simulations, all the SUs are assumed to experience the independent identically distributed Rayleigh fading. We compare the performances of the proposed scheme with that of an existing consensus-based method.

In the context of spectrum sensing [73], the fundamental tradeoff between the missing detection probability and the false alarm probability has different implications. The false alarm can increase the number of the missed opportunity to use the white spaces, while the missing detection can increase the interference to PUs. In cognitive radio network, higher false alarm probability will lead to lower spectrum utilization and higher missing detection probability will result in more interference.

Here, the SUs in the CR-MANET are independent and selfish to share their any information. The proposed mean field scheme is helpful for each SU to make the

decision. While in the consensus-based scheme, the consensus are reached by the 


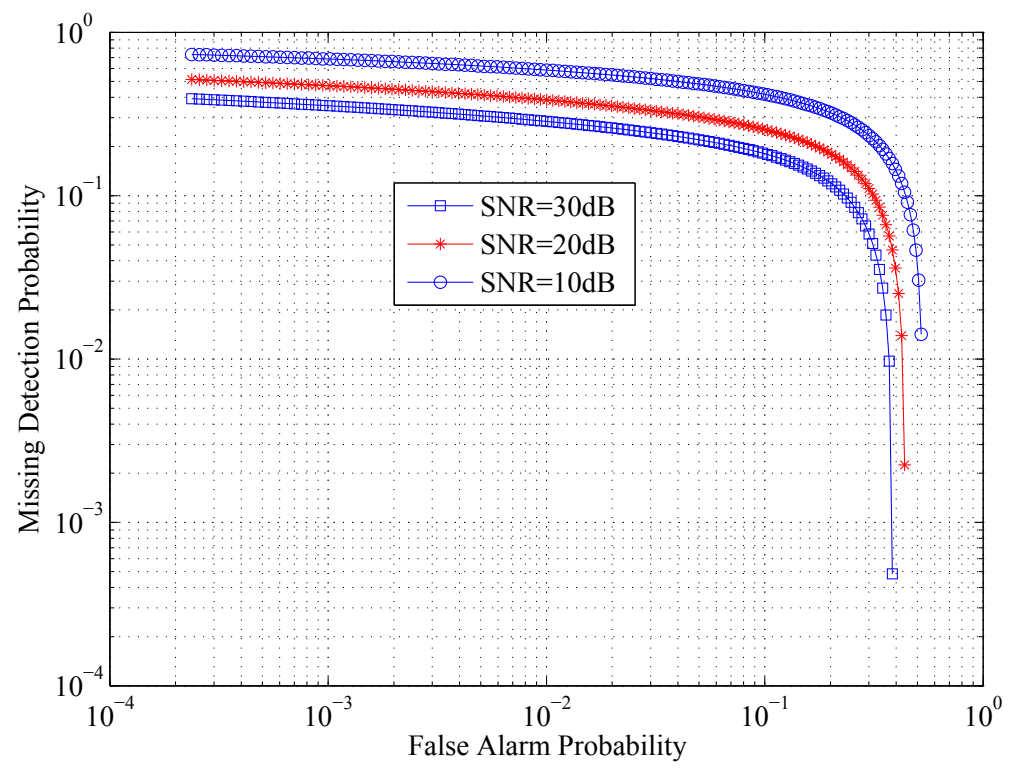

Figure 21: Missing detection probability and false alarm probability.

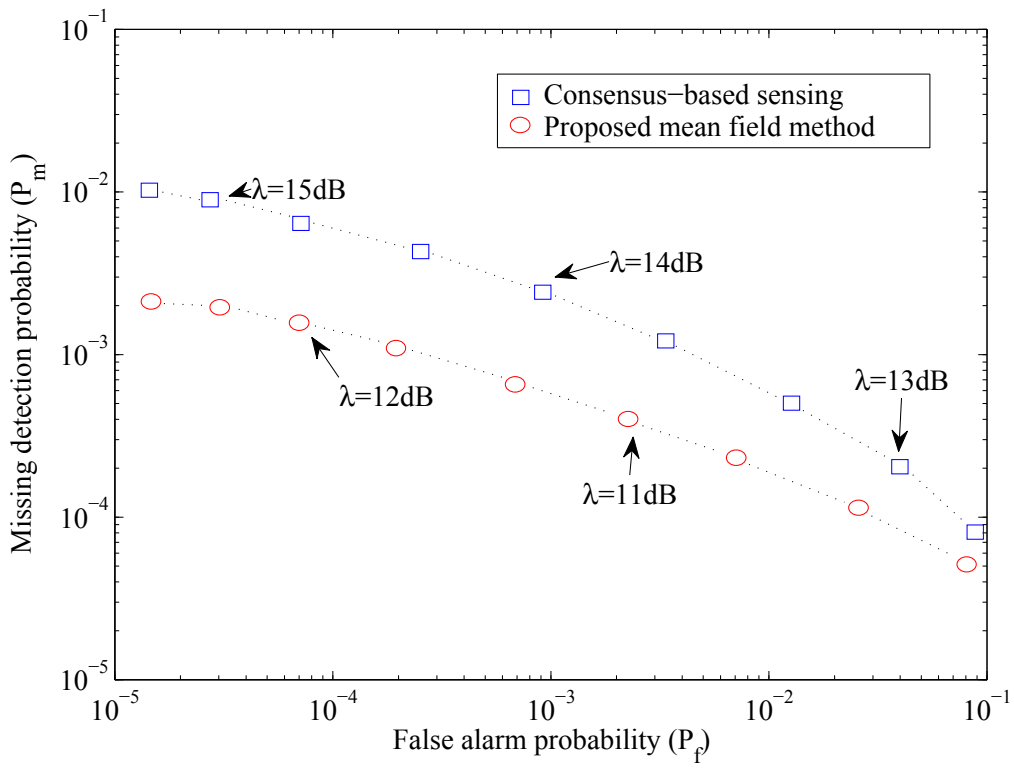

Figure 22: Missing detection probability $P_{m}$ versus false alarm probability $P_{f}$ (when secondary user has the same SNR, i.e., $\bar{\gamma}=10 d B$ ).

nodes which can cooperate with each other. They make the correct decision based on this consensus. 


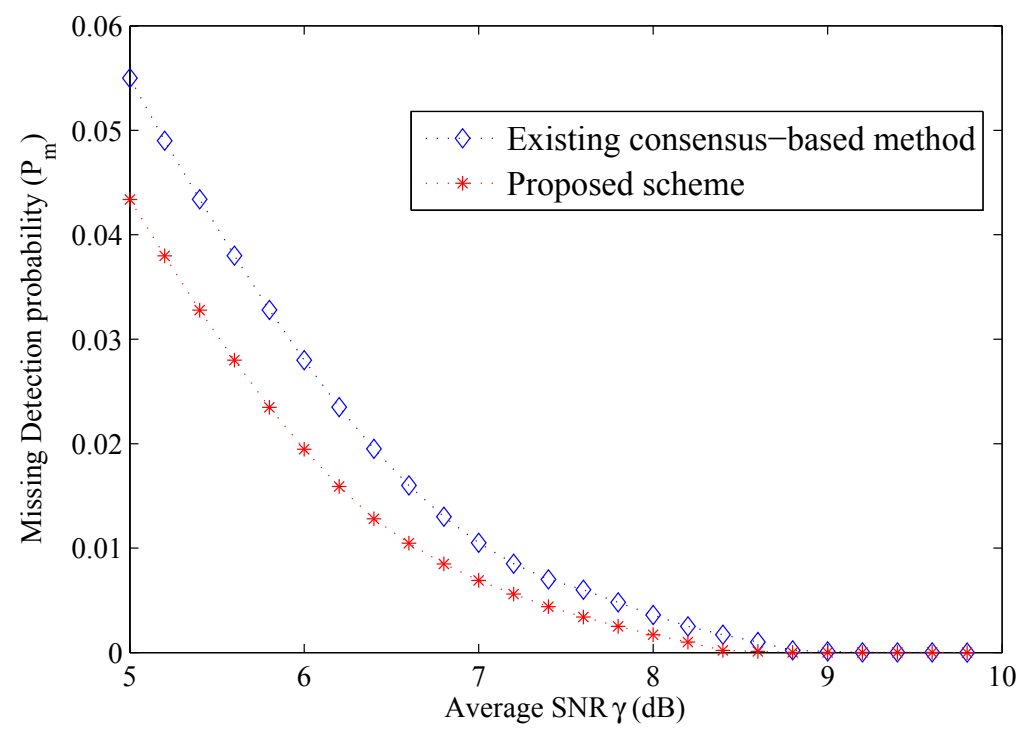

Figure 23: Missing detection probability versus average SNR $\left(P_{f}=10^{-1}, T W=5\right)$.

Here, we compare the false alarm probability and the missing detection probability when the representative player has different SNRs. From Fig. 21, in our proposed scheme we can see that better SNR can lead to the better false alarm probability and better missing detection probability.

Fig. 22 shows the missing detection probability $P_{m}$ versus false alarm probability $P_{f}$. We can see that the proposed scheme has better performance than the existing consensus-based method. The numbers beside the curves are the corresponding thresholds $\lambda$ in decibels. In Fig. 22, the representative secondary user's average SNR is $10 \mathrm{~dB}$. If the threshold $\lambda$ is in the range of $11.2-11.7 \mathrm{~dB}$, both the $P_{m}$ and $P_{f}$ can drop below the probability of $10^{-3}$ for the proposed scheme.

Next, let us examine the performance of missing detection probability $P_{m}$. Fig. 23 shows the relationship of the missing detection probability and the average SNR of SUs. The decision threshold $\lambda$ is chosen to keep $P_{f}=10^{-1}$ and the time-bandwidth product $T W$ is set to 5 . We can see that, from Fig. 23, the proposed scheme can 


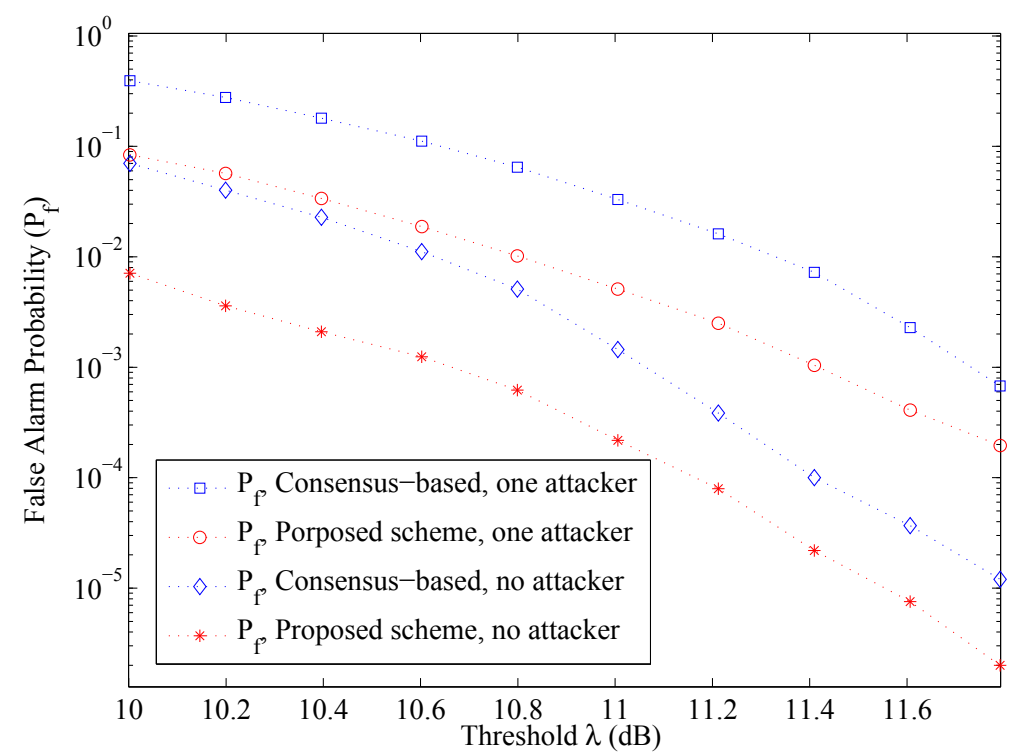

Figure 24: False alarm probability comparison between the proposed scheme and the existing consensus-based scheme.

lead to a significant improvement in terms of the required average SNR for detection. Particularly, if the missing detection probability is kept below 0.01, the existing consensus-based method requires $\bar{\gamma}=7 d B$. While this required average SNR is much higher than that in the proposed scheme, which is $6.7 \mathrm{~dB}$ for the SUs.

Here, we also compare the false alarm probability in the proposed scheme with that in the existing consensus-based scheme. From the Fig. 24, we can see that, when there is an attacker, our proposed scheme can lead to the lower false alarm probabilities. When there is no attacker, the false alarm probability in our proposed scheme is still lower than that in the existing consensus-based method. Particularly, when the threshold $\lambda$ is above $11.6 d B$, the false alarm probability could easily be kept below 0.001 in our proposed scheme. 


\subsection{Summary}

Security is an important issue in CR-MANETs. In this chapter, we proposed a novel mean field game theoretic approach for security in CR-MANETs to model the interactions among a malicious node and a large number of legitimate nodes. Unlike the existing works on security game modeling, the proposed scheme can enable an individual node in CR-MANETs to make distributed security defence decisions. Both security requirement and system resources were considered in the proposed scheme.

The simulation results demonstrated that, with the optimal strategy, the legitimate nodes can choose distributed actions intelligently to reduce their energy consumption. The performances of the CR-MANET can be improved significantly. In

our future work, we will consider our proposed scheme with multiple attackers in CR-MANETs. 


\section{Chapter 6}

\section{Conclusions and Future Work}

\subsection{Conclusions}

In this thesis, we proposed a novel mean field game theoretic approach for security in both the MANET and the CR-MANET to model the interactions among a malicious node and a large number of legitimate nodes. Unlike the existing works on security game modeling, the proposed scheme can enable an individual node in MANETs or CR-MANETs to make distributed security defence decisions. Both security requirement and system resources were considered in the proposed scheme.

To overcome the fundamental complexity, we proposed an approximation method, using a limiting process $\theta(t)$ to instead the random measure process $I^{(N)}(t)$ in MANETs and CR-MANETs. We designed the updating rule for $\theta(t)$ and the error estimate on the mean field approximation was analyzed. To obtain the distributed optimal defending strategy in MANETs, the mixed strategies and the law of state transition for the major player (attacker) were discussed based on the mean field modeling and analysis. The major player we considered, was a smart attacker who can load the attack dynamically for the maximum reward. The interactions between the major player and a representative minor player were modeled and presented using 
the cost functions and state transition matrix.

For the security problem in CR-MANETs, we considered a very critical situation for the tactical CR-MANETs when using the proposed scheme. The jamming attack was loaded by a smart attacker in this research to disrupt the communication among the secondary users. However, when the primary user was using the spectrum, the attacker did not load the attack for the heavy punishment.

We designed and performed the simulations to evaluate the proposed mean field game theoretical method in these two kinds of networks. The simulation results demonstrated that, with the optimal strategy, the legitimate nodes can choose distributed actions intelligently to reduce their energy consumption and security value loss in MANETs. The average lifetime of the MANET can be improved significantly and the compromising probability can be reduced as well. In CR-MANETs, we also considered the CR-specific performance measures, such as the false alarm probability and the missing detection probability. The simulation results indicated that, using the proposed scheme, the performances of the CR-MANET can be improved significantly as well.

\subsection{Future Work}

In future research, we would like to design more robust game theoretic approach for security and resource consumption to cope with the attack initiated by multiple attackers in MANETs and CR-MANETs. This new game theoretic approach should be applicable to handle security problems in MANETs or CR-MANETs, no matter the number of attackers is known or unknown. New updating rules for the limiting process $\theta(t)$ should be proposed based on the attacks loaded by a number of multiple attackers.

We would also like to consider the proposed game theoretic approach for routing 
nodes selection in mobile ad-hoc networks. Since the existence of noncooperative nodes would drop off arrival packets which are sent from the source or malicious nodes.

Last but not the least, we would like to devise more accurate and pragmatic utility functions to depict the interactions between a large number of defenders and multiple attackers. 


\section{List of References}

[1] K. J. R. Liu and B. Wang, Cognitive Radio Networking and Security. Cambridge, UK: Cambridge University Press, 2010.

[2] M. Carvalho, "Security in mobile ad hoc networks," IEEE Security and Privacy, vol. 6, pp. 72-75, Mar 2008.

[3] H. Yang, H. Luo, F. Ye, S. Lu, and L. Zhang, "Security in mobile ad hoc networks: challenges and solutions," IEEE Trans. Wireless Commun., vol. 11, pp. 38-47, Feb 2004.

[4] X. Liao, D. Hao, and K. Sakurai, "Classification on attacks in wireless ad hoc networks: A game theoretic view," in Proc. Yth Int'l Conf. Networked Computing and Advanced Information Management (NCM), (Gyeongju, Korea), pp. 144149, Jun 2011.

[5] R. Ramamoorthy, F. R. Yu, H. Tang, and P. C. Mason, "Combined authentication and quality of service in cooperative communication networks," in Proc. Embedded and Ubiquitous Computing (EUC), (Hong kong, China), pp. 566-571, Dec 2010.

[6] C. E. Perkins, Ad Hoc Networking. Boston : Addison-Wesley, 2001.

[7] Hoboken, Mobile Ad hoc Networking. NJ : John Wiley, 2004.

[8] S. Basagni, M. Conti, S. Giordano, and I. Stojmenovic, Mobile Ad Hoc Networking: The Cutting Edge Directions. Wiley-IEEE Press, 2013.

[9] Y. Wang, F. R. Yu, M. Huang, A. Boukerche, and T. Chen, "Securing vehicular ad hoc networks with mean field game theory," in Proc. ACM DIVANet'13, (New York, NY, USA), pp. 55-60, ACM, 2013. 
[10] M. Al-kahtani, "Survey on security attacks in vehicular ad hoc networks (vanets)," in Proc. Signal Processing and Communication Systems (ICSPCS), pp. 1-9, Dec 2012.

[11] D. Watkins and C. Scott, "Methodology for evaluating the effectiveness of intrusion detection in tactical mobile ad-hoc networks," in Proc. IEEE WCNC'04, vol. 1, pp. 622-627, Mar 2004.

[12] B. Sun, L. Osborne, Y. Xiao, and S. Guizani, "Intrusion detection techniques in mobile ad hoc and wireless sensor networks," IEEE Trans. Wireless Commun., vol. 14, pp. 56-63, Oct 2007.

[13] J. Kim, D. Kim, S. M. Jung, C. Lee, D. Lim, S. Hong, and S. Yoo, "Development of mobile ad hoc network for emergency telemedicine service in disaster areas," in Proc. New Trends in Information and Service Science (NISS), pp. 1291-1296, Jun 2009.

[14] I. Rubin and R. Zhang, "Performance behavior of unmanned vehicle aided mobile backbone based wireless ad hoc networks," in Proc. 57th Int'l Conf. Vehicular Technology Conference (VTC), vol. 2, pp. 955-959, Apr 2003.

[15] R. Bruno, M. Conti, and E. Gregori, "Wlan technologies for mobile ad hoc networks," in Proc. 34th Int'l Conf. System Sciences, pp. 1-11, Jan 2001.

[16] F. Zeiger, N. Kraemer, M. Sauer, and K. Schilling, "Challenges in realizing adhoc networks based on wireless lan with mobile robots," in Proc. 6th Int'l Conf. Modeling and Optimization in Mobile, Ad Hoc, and Wireless Networks, pp. 632639, Apr 2008.

[17] Y. Wang, G. Attebury, and B. Ramamurthy, "A survey of security issues in wireless sensor networks," IEEE Communications Surveys and Tutorials, vol. 8, pp. 2-23, 2006.

[18] W. Stallings, Cryptography and network security. Prentice Hall, second ed., 1999.

[19] G. Schäfer, Security in Fixed and Wireless Networks: An Introduction to securing data communications. John Wiley \& Sons, 2004.

[20] S. Maity, P. Bera, and S. Ghosh, "An access control framework for semiinfrastructured ad hoc networks," in Proc. 2nd Int'l Conf. Computer Technology and Development (ICCTD), pp. 708-712, Nov 2010. 
[21] H. Yu, R. Chen, and Z. Du, "Message oriented middleware support for mobile ad hoc data sharing," in Proc. 34th Int'l Conf. Computer Software and Applications Conference Workshops (COMPSACW), pp. 305-310, Jul 2010.

[22] J. Liu, F. R. Yu, C.-H. Lung, and H. Tang, "Optimal combined intrusion detection and biometric-based continuous authentication in high security mobile ad hoc networks," IEEE Trans. Wireless Commun., vol. 8, pp. 806-815, Feb 2009.

[23] S. Bu, F. R. Yu, X. P. Liu, and H. Tang, "Structural results for combined continuous user authentication and intrusion detection in high security mobile ad-hoc networks," IEEE Trans. Wireless Commun., vol. 10, pp. 3064-3073, Sep 2011.

[24] Y. Zhang, W. Lee, and Y. Huang, "Intrusion detection techniques for mobile wireless networks," Mobile Net. and App., vol. 9, pp. 45-56, Sep 2003.

[25] A. Mishra, K. Nadkarni, and A. Patcha, "Intrusion detection in wireless ad hoc networks," IEEE Trans. Wireless Commun., vol. 11, pp. 48-60, Feb 2004.

[26] Y. Zhang and W. Lee, "Intrusion detection in wireless ad hoc networks," in Proc. ACM MOBICOM'00, pp. 275-283, 2000.

[27] X. Liu, Z. Fang, and L. Shi, "Securing vehicular ad hoc networks," in Proc. Pervasive Computing and Applications, pp. 424-429, 2007.

[28] C. Zhang, X. Lin, R. Lu, and P.-H. Ho, "Raise: An efficient rsu-aided message authentication scheme in vehicular communication networks," in Proc. Communications (ICC), pp. 1451-1457, 2008.

[29] G. Guette and B. Ducourthial, "On the sybil attack detection in vanet," in Proc. Mobile Adhoc and Sensor Systems, pp. 1-6, 2007.

[30] M. Raya and J. P. Hubaux, "The security of vehicular ad hoc networks," in Proc. the 3rd ACM workshop on Security of ad hoc and sensor networks (SASN'05), (New York, NY, USA), 2005.

[31] S. Safi, A. Movaghar, and M. Mohammadizadeh, "A novel approach for avoiding wormhole attacks in vanet," in Proc. Computer Science and Engineering(WCSE), vol. 2, pp. 160-165, 2009.

[32] J. Sun and Y. Fang, "A defense technique against misbehavior in vanets based on threshold authentication," in Proc. MILCOM'08, pp. 1-7, 2008. 
[33] S. S. Manvi, M. S. Kakkasageri, and D. G. Adiga, "Message authentication in vehicular ad hoc networks: Ecdsa based approach," in Proc. Future Computer and Communication (ICFCC), pp. 16-20, 2009.

[34] A. Gueye, A Game Theoretical Approach to Communication Security. PhD thesis, University of California at Berkeley, Berkeley, CA, USA, 2011.

[35] X. Liang and Y. Xiao, "Game theory for network security," IEEE Communications Surveys and Tutorials, vol. 15, no. 1, pp. 472-486, 2013.

[36] S. Buchegger and T. Alpcan, "Security games for vehicular networks," in Proc. Communication, Control, and Computing, pp. 244-251, 2008.

[37] T. Alpcan and S. Buchegger, "Security games for vehicular networks," IEEE Trans. Mobile Computing, vol. 10, no. 2, pp. 280-290, 2011.

[38] J. Mitola, Cognitive radio: an integrated agent architecture for software defined radio. PhD thesis, KTH Royal Institute of Technology, Stockholm, Sweden, 2000.

[39] A. Attar, H. Tang, A. Vasilakos, F. Yu, and V. Leung, "A survey of security challenges in cognitive radio networks: Solutions and future research directions," Proceedings of the IEEE, vol. 100, pp. 3172-3186, Dec 2012.

[40] H. Tembine, R. Tempone, and P. Vilanova, "Mean field games for cognitive radio networks," in Proc. American Control Conference (ACC), pp. 6388-6393, Jun 2012.

[41] B. Wang, Y. Wu, K. Liu, and T. Clancy, "An anti-jamming stochastic game for cognitive radio networks," Selected Areas in Communications, vol. 29, pp. 877889, Apr 2011.

[42] D. Cabric, S. Mishra, and R. Brodersen, "Implementation issues in spectrum sensing for cognitive radios," in Proc. Signals, Systems and Computers, vol. 1, pp. 772-776, Nov 2004.

[43] Z. Li, F. R. Yu, and M. Huang, "Distributed spectrum sensing in cognitive radio networks," in Proc. IEEE WCNC'09, pp. 1-5, Apr 2009.

[44] O. Morgenstern and J. Von Neumann, Theory of Games and Economic Behavior. Princeton University Press, third ed., May 1944.

[45] J. Nash, "Two person cooperative games," Econometrica, vol. 21, pp. 128-140, Jan 1953. 
[46] M. Felegyhazi and J. Hubaux, "Game theory in wireless networks: A Tutorial," tech. rep., EPFL, 2006.

[47] N. Nisan, T. Roughgarden, E. Tardos, and V. V. Vazirani, Algorithmic Game Theory. Cambridge University Press, 2007.

[48] D. Zheng, "A game theoretic approach for security and quality of service (qos) co-design in cooperative wireless communication networks," Master's thesis, Carleton University, Ottawa, ON, Canada, 2012.

[49] R. Gibbons, Game Theory for Applied Economists. Princeton University Press, 1992.

[50] Y. Wang, F. Yu, H. Tang, and M. Huang, "A mean field game theoretic approach for security enhancements in mobile ad hoc networks," IEEE Trans. Wireless Commun., vol. 13, pp. 1616-1627, Mar 2014.

[51] H. Bedi, S. Roy, and S. Shiva, "Game theory-based defense mechanisms against ddos attacks on tcp/tcp-friendly flows," in Proc. Computational Intelligence in Cyber Security (CICS), pp. 129-136, 2011.

[52] A. Patcha and J. M. Park, "A game theoretic formulation for intrusion detection in mobile ad hoc networks," International Journal of Network Security, vol. 2, no. 2, pp. 131-137, 2006.

[53] E. A. Panaousis and C. Politis, "A game theoretic approach for securing AODV in emergency mobile ad hoc networks," in Proc. 34th IEEE Conference on Local Computer Networks (LCN), vol. 53, (Zurich, Switzerland), pp. 985-992, Oct 2009.

[54] J. Omic, A. Orda, and P. Van Mieghem, "Protecting against network infections: A game theoretic perspective," in Proc. IEEE INFOCOM'09, pp. 1485-1493, 2009.

[55] N. Santosh, R. Saranyan, K. Senthil, and V. Vetriselvi, "Cluster based cooperative game theory approach for intrusion detection in mobile ad-hoc grid," in Proc. 16th Int'l Conf. Advanced Computing and Communications (ADCOM), pp. 273-278, Dec 2008.

[56] A. Fourati and K. Al Agha, "An ids first line of defense for ad hoc networks," in Proc. IEEE WCNC'O', pp. 2619-2624, Mar 2007. 
[57] F. Li, Y. Yang, and J. Wu, "Attack and flee: Game-theory-based analysis on interactions among nodes in MANETs," IEEE Trans. Syst., Man, Cybern. (B), vol. 40, pp. 612-622, Jun 2010.

[58] G. Quer, F. Librino, L. Canzian, L. Badia, and M. Zorzi, "Using game theory and bayesian networks to optimize cooperation in ad hoc wireless networks," in Proc. Computer Communications (ICC), pp. 5178-1584, June 2012.

[59] M. Huang, P. Caines, and R. Malhame, "The NCE (mean field) principle with locality dependent cost interactions," IEEE Trans. Auto. Control, vol. 55, pp. 2799-2805, Dec 2010.

[60] M. Y. Huang, "Mean field stochastic games with discrete states and mixed players," in Proc. GameNets, (Vancouver, BC, Canada), May 2012.

[61] M. Huang, "Mean field capital accumulation games: The long time behavior," in Proc. 52nd Int'l Conf. Decision and Control (CDC), pp. 2499-2504, Dec 2013.

[62] M. Huang, R. P. Malham, and P. E. Caines, "Large population stochastic dynamic games: closed-loop mckean-vlasov systems and the nash certainty equivalence principle," Communications in Information and Systems, vol. 6, pp. 221-252, Mar 2006.

[63] S. L. Nguyen and M. Huang, "Mean field lqg games with mass behavior responsive to a major player," in Proc. 51st Int'l Conf. Decision and Control (CDC), pp. 5792-5797, Dec 2012.

[64] J. Huang and M. Huang, "Mean field lqg games with model uncertainty," in Proc. 52nd Int'l Conf. Decision and Control (CDC), pp. 3103-3108, Dec 2013.

[65] D. Zheng, H. Tang, and F. R. Yu, "Game theoretic approach for security and quality of service (QoS) co-design in MANETs with cooperative communications," in Proc. MILCOM'12, (Maui, HI), pp. 1-6, Oct 2012.

[66] T. Alpcan and T. Basar, "A game theoretic approach to decision and analysis in network intrusion detection," in Proc. 42nd IEEE Conference on Decision and Control, (Maui, HI), pp. 2595-2600, Dec 2003.

[67] C. Liang and K. R. Dandekar, "Power management in MIMO ad hoc networks: A game-theoretic approach," IEEE Trans. Wireless Commun., vol. 6, pp. 28662882, Apr 2007. 
[68] H. Zhang, O. Kreidl, B. DeCleene, J. Kurose, and X. Ni, "Security analysis of the bootstrap protocol for deny-by-default mobile ad-hoc networks," in Proc. MILCOM'09, (Boston, MA), Oct 2009.

[69] P. Hiremath and S. M. Joshi, "Energy efficient routing protocol with adaptive fuzzy threshold energy for manets," International Journal of Computer Networks and Wireless Communications (IJCNWC), vol. 2, pp. 402-407, Jun 2012.

[70] I. F. Akyildiz, W. Y. Lee, M. C. Vuran, and S. Mohanty, "Next generation/dynamic spectrum access/cognitive radio wireless networks: A survey," Computer Networks, vol. 50, pp. 2127-2159, 2006.

[71] F. R. Yu, H. Tang, M. Huang, Z. Li, and P. Mason, "Defense against spectrum sensing data falsification attacks in mobile ad hoc networks with cognitive radios," in Proc. MILCOM'09, pp. 1-7, Oct 2009.

[72] T. Sood and M. Singh, "On the performance of detection based spectrum sensing for cognitive radio," Electronics and Communication Technology, pp. 140-143, Sep 2011.

[73] H. Tang, F. R. Yu, M. Huang, and Z. Li, "Distributed consensus-based security mechanisms in cognitive radio mobile ad hoc networks," IET Commun., vol. 6, pp. 974-983, May 2012. 


\section{Appendix A}

\section{Simulation Programs}

mdp_solution_major.m

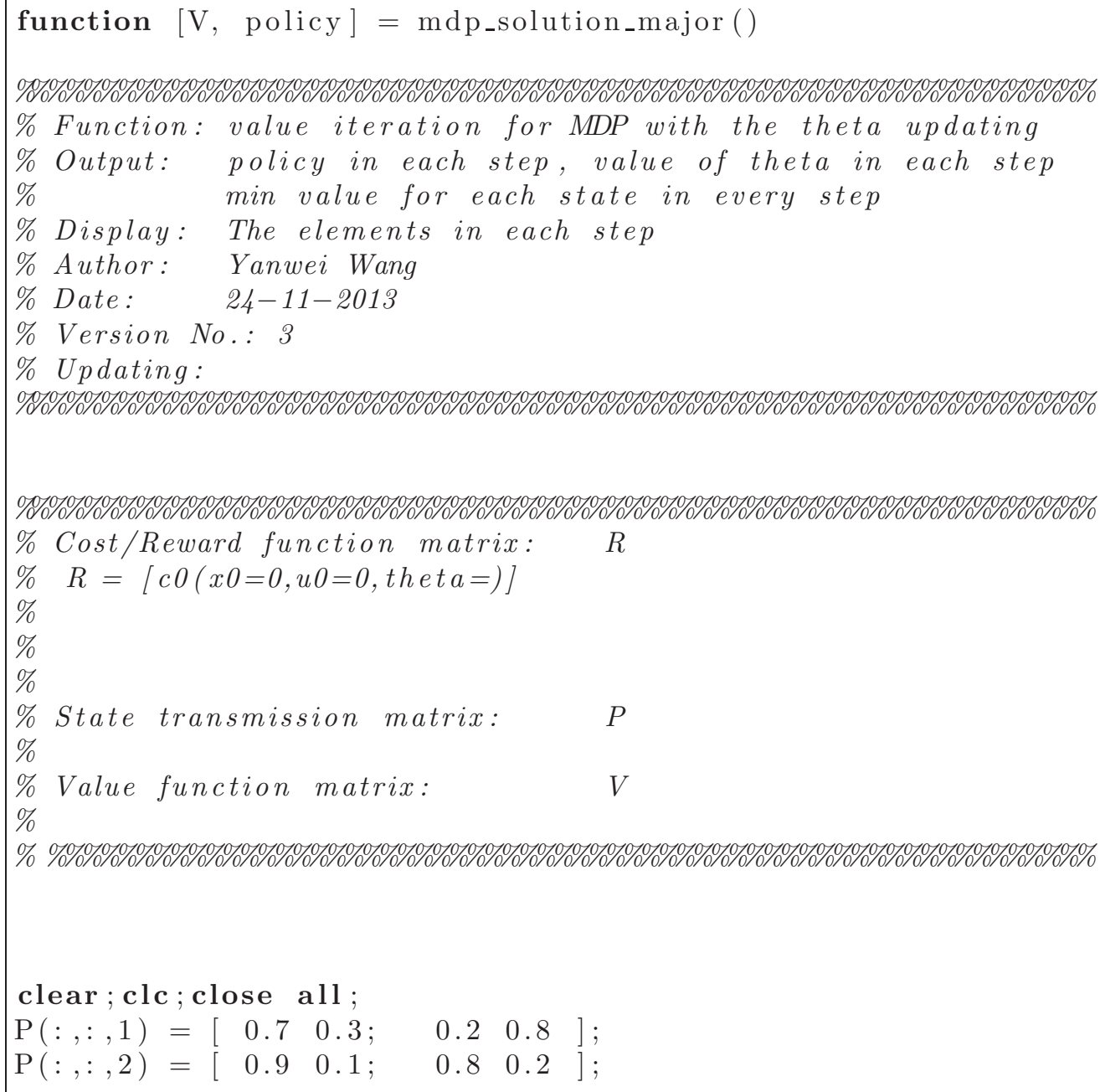




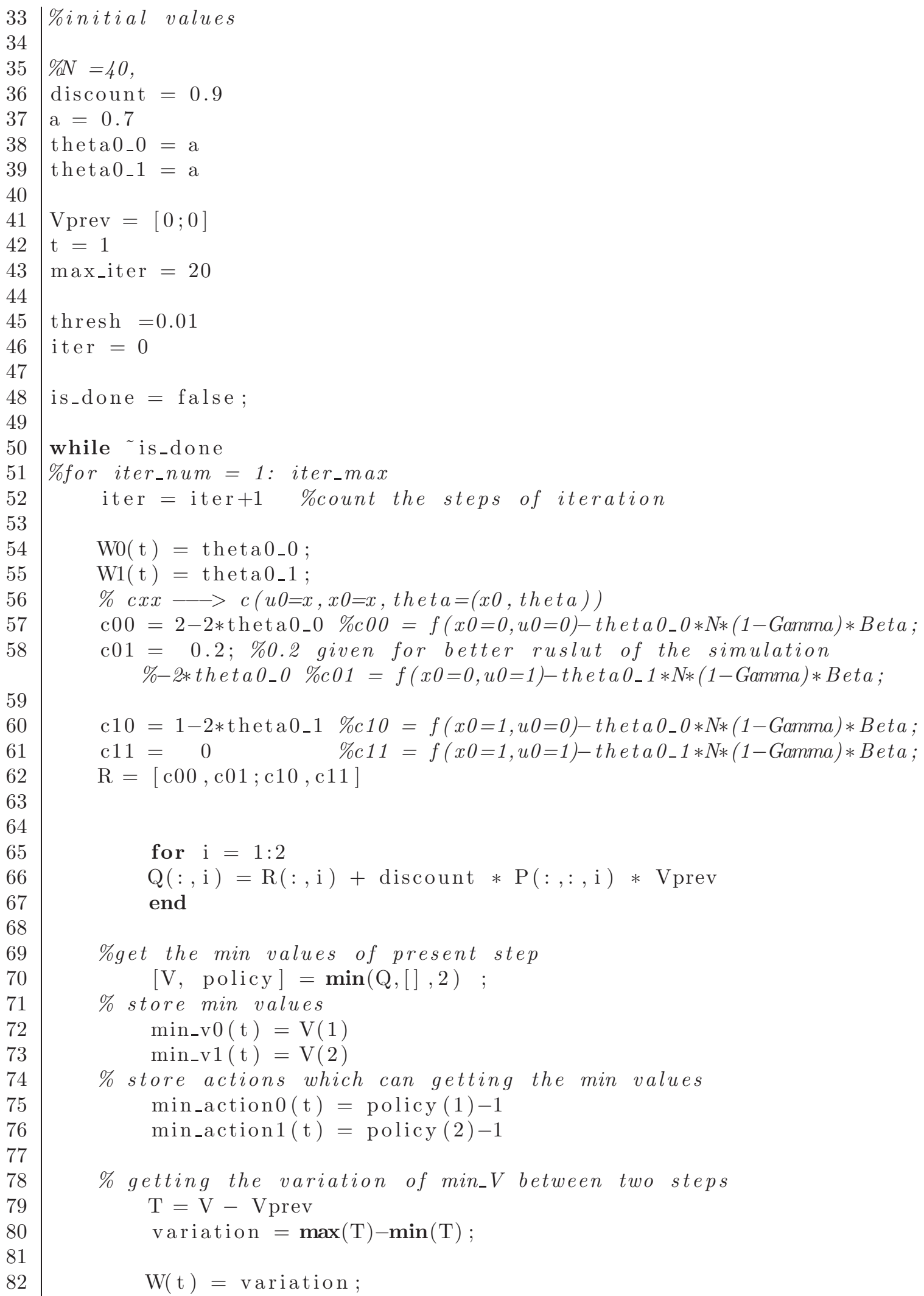




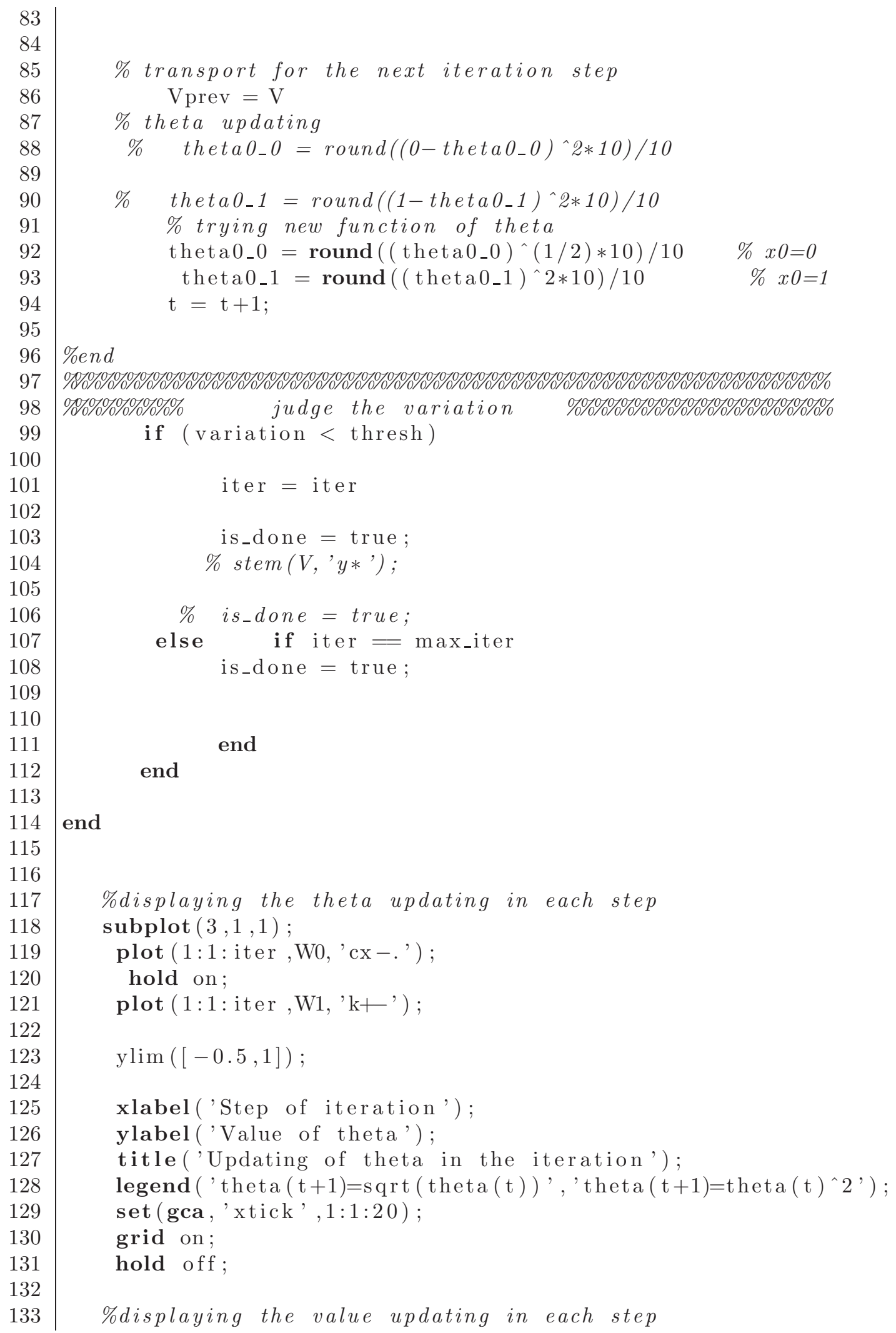


134

subplot $(3,1,2)$;

plot (1:1: iter, min_v0, 'ro-');

hold on;

plot $(1: 1$ : iter , min_v1, 'b*-') ;

$\% \lim ([-0.5,1]) ;$

xlabel ('Step of iteration');

ylabel ('Minimum value of $\mathrm{V}(\mathrm{x} 0$, theta)');

title ('Values of $\mathrm{V}(\mathrm{x} 0$, theta) of the iteration');

legend ('v $(\mathrm{x} 0=0$, theta )', 'v $(\mathrm{x} 0=1$, theta )' );

set (gca,' xtick', 1:120);

grid on;

hold off;

subplot $(3,1,3)$;

$\%$ displaying the actions of each step

plot (1:1: iter, min_action 0, 'go-') ;

hold on ;

plot (1:1: iter,min_action 1, 'm---');

$y \lim ([-0.5,1.5])$;

xlabel ('Step of iteration');

ylabel ('Actions');

title ('Policy of the iteration') ;

legend (' $\mathrm{u} 0 \mid \mathrm{x} 0=0$ ', ' $\mathrm{u} 0 \mid \mathrm{x} 0=1$ ') ;

set (gca,'xtick', 1:1:20);

grid on;

hold on ;

$\% \operatorname{plot}\left(i\right.$, min_action $\left._{-}(2 i+2),{ }^{\prime} b *-\right)$

\%test function

mdp_solution_minor.m

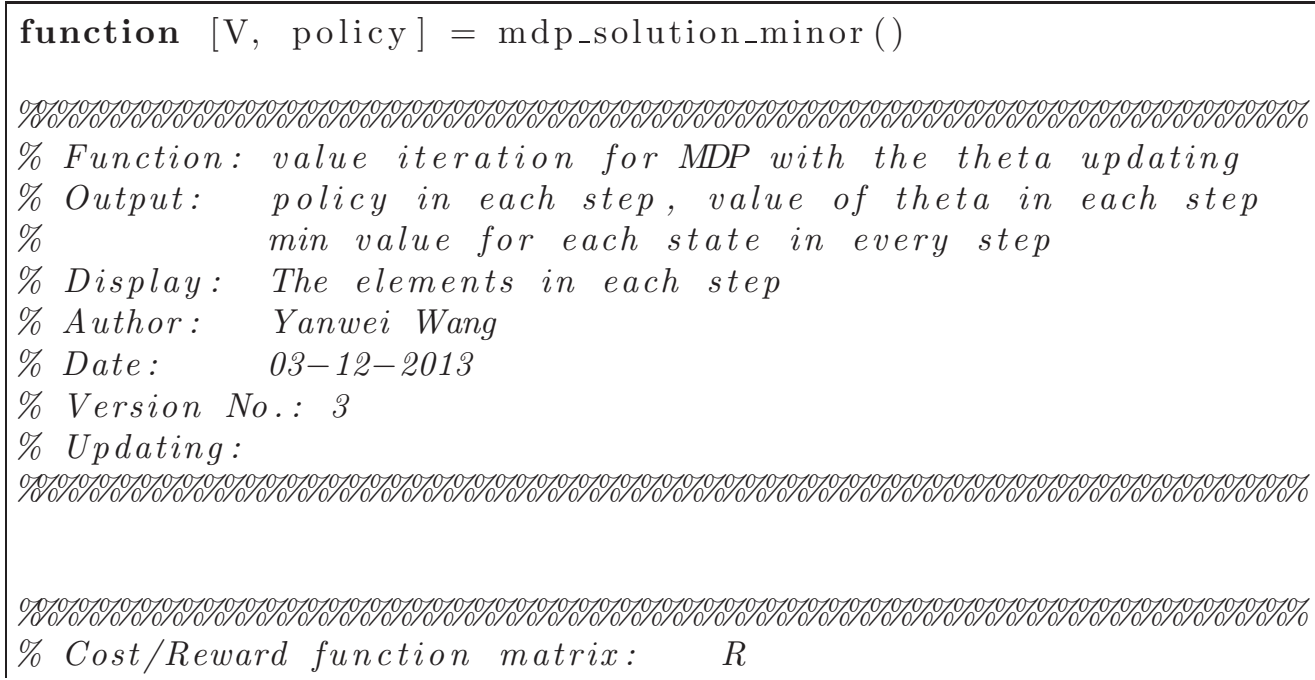




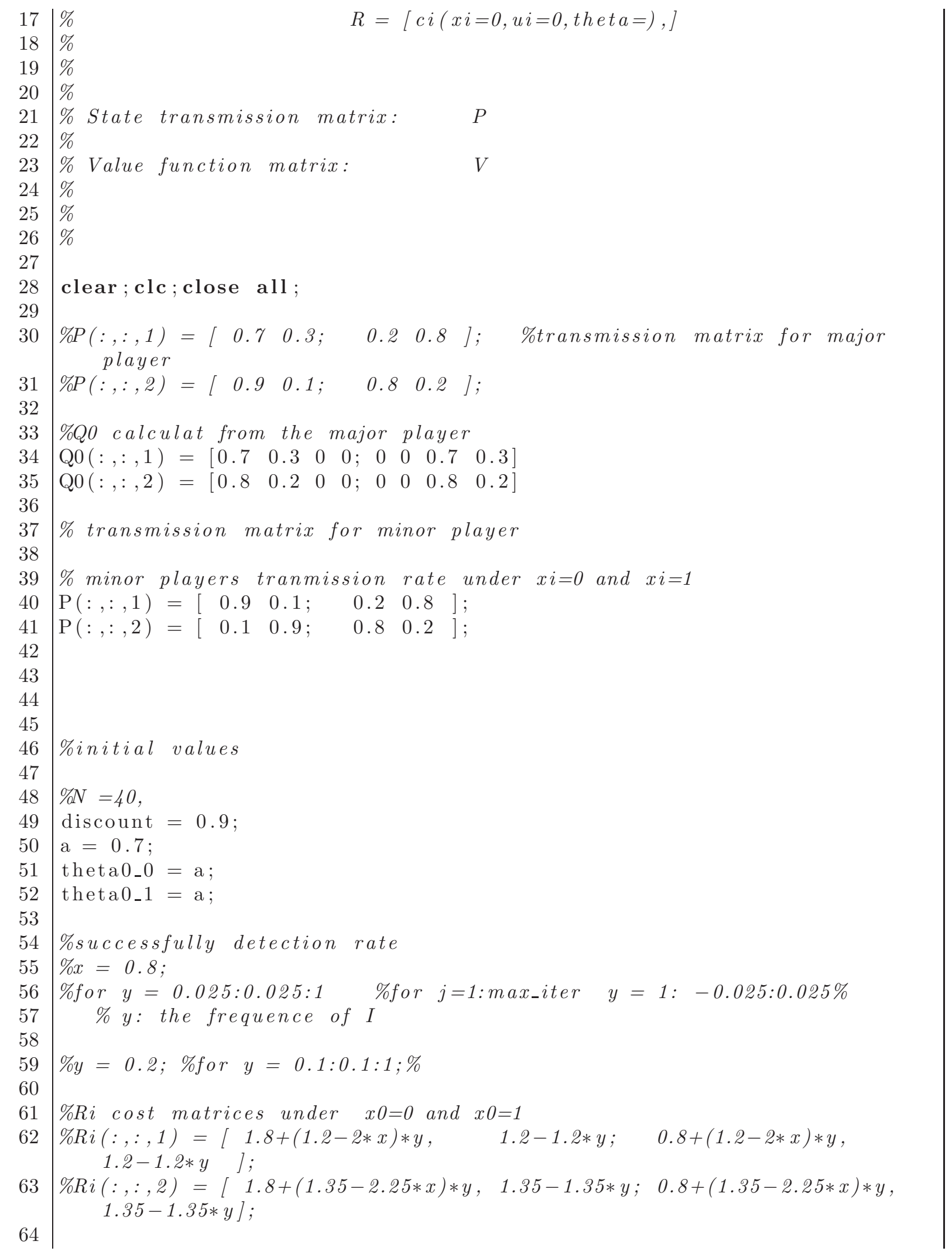




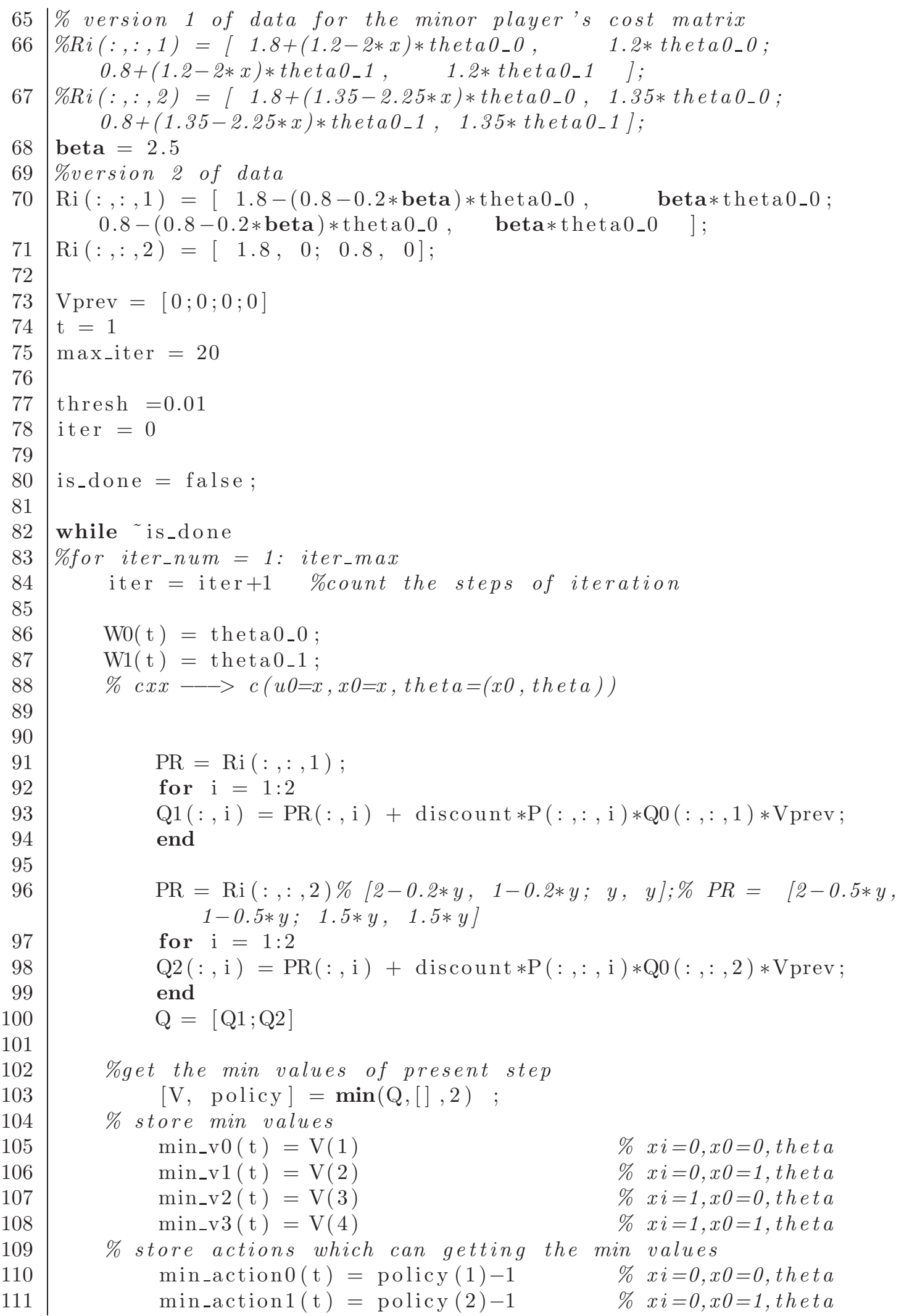




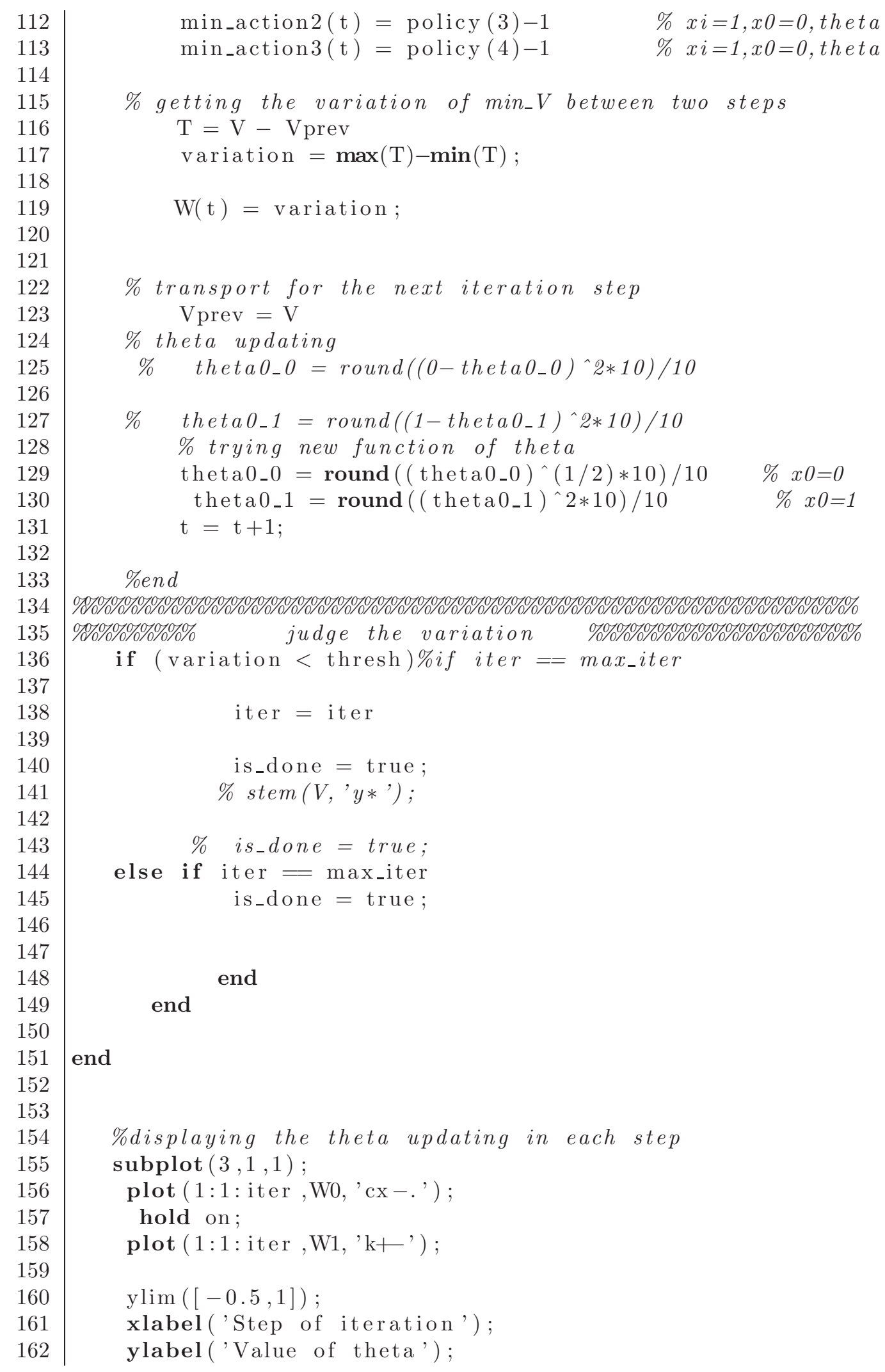


title ('Updating of theta in the iteration');

legend ('theta $(\mathrm{t}+1)=\operatorname{sqrt}(\mathrm{theta}(\mathrm{t}))$ ', ' theta $(\mathrm{t}+1)=\operatorname{theta}(\mathrm{t}))^{\wedge} 2$ ') ;

set (gca,'xtick', 1:1:20);

grid on ;

hold off;

\%displaying the value updating in each step

subplot $(3,1,2)$;

plot (1:1: iter, min_v0, 'ro-');

hold on ;

plot (1:1: iter, min_v1, 'b*-') ;

hold on ;

plot (1:1: iter , min_v2, 'gx-') ;

hold on ;

plot $(1: 1$ : iter , min_v3, 'k+') ;

$\% \operatorname{ylim}([-0.5,1])$;

xlabel ('Step of iteration');

ylabel('Minimum value of W(xi, x0, theta)');

title ('Values of W(xi, $x 0$, theta) of the iteration') ;

legend (' $\mathrm{w}(\mathrm{xi}=0, \mathrm{x} 0=0$, theta $)$ ', ' $\mathrm{w}(\mathrm{xi}=1, \mathrm{x} 0=0$, theta $)$ ',

'w $(\mathrm{xi}=0, \mathrm{x} 0=1$, theta $)$ ', 'w( $\mathrm{xi}=1, \mathrm{x} 0=1$, theta $)$ ') ;

$\%$ set (gca, 'xtick', 1:1:20);

$\%$ grid on;

$\%$ hold off;

subplot $(3,1,3)$;

$\%$ displaying the actions of each step

plot (1:1:iter, min_action0, 'ro-') ;

hold on ;

plot (1:1: iter , min_action 1, 'b*-') ;

hold on ;

plot (1:1: iter, min_action 2, 'gx-') ;

hold on;

plot (1:1: iter, min_action 3, 'k+' ) ;

$y \lim ([-0.5,1.5])$;

xlabel ('Step of iteration') ;

ylabel ('Actions');

title ('Policy of the iteration') ;

legend (' ui $\mid x i=0, x 0=0$ ', ' ui $\mid x i=1, x 0=0$ ', ' ui $\mid x i=0, x 0=1$ ', ' ui $\mid x i=1, x 0=1$ ') ; set (gca,'xtick', 1:1:20);

grid on;

hold on;

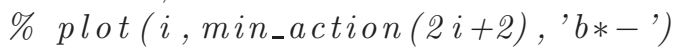

